\title{
ORIENTED FLIP GRAPHS, NONCROSSING TREE PARTITIONS, AND REPRESENTATION THEORY OF TILING ALGEBRAS
}

\author{
ALEXANDER GARVER \\ Université du Québec à Montréal, Montréal, Canada \\ e-mail:alexander.garver@gmail.com \\ and THOMAS MCCONVILLE \\ Mathematical Sciences Research Institute, Berkeley, CA, USA \\ e-mail: thomasmcconvillea@gmail.com
}

(Received 16 March 2018; revised 29 November 2018; accepted 7 January 2019; first published online 7 February 2019)

\begin{abstract}
The purpose of this paper is to understand lattices of certain subcategories in module categories of representation-finite gentle algebras called tiling algebras, as introduced by Coelho Simões and Parsons. We present combinatorial models for torsion pairs and wide subcategories in the module category of tiling algebras. Our models use the oriented flip graphs and noncrossing tree partitions, previously introduced by the authors, and a description of the extension spaces between indecomposable modules over tiling algebras. In addition, we classify two-term simple-minded collections in bounded derived categories of tiling algebras. As a consequence, we obtain a characterization of $\mathbf{c}$-matrices for any quiver mutation-equivalent to a type $A$ Dynkin quiver.
\end{abstract}

2010 Mathematics Subject Classification. Primary 16G20; Secondary 05E10 and $18 \mathrm{E} 40$

1. Introduction. A tiling algebra $\Lambda_{T}=\mathbb{k} Q_{T} / I_{T}$ is defined by the data of a tree $T$ embedded in a disk. They are a class of representation-finite gentle algebras that were recently introduced in [12]. These algebras also form a subclass of the algebras of partial triangulations introduced in [13]. Examples of tiling algebras include Jacobian algebras [15] of type $A$ and $m$-cluster-tilted algebras [30] of type $A$, both of which naturally arise in the study of cluster algebras [16] and in the additive categorification of cluster algebras $[6,7]$.

In a separate paper [21], we introduced two lattices associated with the tree $T$ : the oriented flip graph of $T$, denoted $\overrightarrow{F G}(T)$, and the lattice of noncrossing tree partitions of $T$, denoted $\operatorname{NCP}(T)$. The former can be regarded as a directed graph whose vertices correspond to partial triangulations of a disk and whose edges correspond to exchanging single arcs in the corresponding partial triangulations. The latter is a generalization of the classical noncrossing set partitions of the set $\{1,2, \ldots, n\}$ where $n$ is the number of nonleaf vertices of $T$.

In [21], we studied a canonical bijection between $\overrightarrow{F G}(T)$ and $\operatorname{NCP}(T)$ defined by their lattice structures. In this paper, we show that the two lattices and the canonical bijection admit a representation-theoretic interpretation. Namely, we present combinatorial models for the torsion pairs and the wide subcategories in the module category of $\Lambda_{T}$ using the 
oriented flip graph of $T$ and the noncrossing tree partitions of $T$, respectively. In particular, we prove the following:

- the lattice of torsion-free classes, denoted torsf $\left(\Lambda_{T}\right)$ (resp., of torsion classes, denoted tors $\left(\Lambda_{T}\right)$ ), is isomorphic to $\overrightarrow{F G}(T)$ (resp., $\overrightarrow{F G}(T)^{\text {op }}$ ) (see Theorem 6.4),

- the lattice of wide subcategories of $\Lambda_{T}$, denoted wide $\left(\Lambda_{T}\right)$, is isomorphic to $\operatorname{NCP}(T)$ (see Theorem 7.1).

For any algebra with finitely many torsion classes, a canonical bijection between wide subcategories and torsion classes, thus also torsion-free classes, was given in [29]. Their bijection is analogous to a previously discovered correspondence in [24] between wide subcategories and torsion classes for the path algebra of any acyclic quiver.

In [19], we proved that the lattice of torsion classes of any algebra is semidistributive. This result was strengthened in [14], where this lattice was proved to be completely congruence-uniform. We leave the definitions of these lattice properties to these papers. In the case where these lattices are finite, congruence-uniformity provides a bijection with another poset known as the shard intersection order. Combining the results of this paper with [21] shows that the lattice-theoretic bijection agrees with the bijection defined in [29] for tiling algebras. We believe that this can be extended to any algebra with finitely many torsion classes, but this is beyond the scope of this paper.

We also combinatorially describe all two-term simple-minded collections in the bounded derived category of $\Lambda_{T}$ (see Theorem 8.4). An important application of the latter is a classification of c-matrices of quivers that are mutation-equivalent to type $A$ Dynkin quivers (see Theorem 9.1). This classification is similar to one obtained in [36, Theorem 1.1] for acyclic quivers and to the classification found in [18, Theorem 3.15] for type $A$ Dynkin quivers.

This paper was motivated in part by the bijections discovered in [5] among various combinatorial and representation-theoretic objects associated with a quiver with potential with a finite-dimensional Jacobian algebra. The collections they consider include torsion classes, two-term simple minded collections, c-matrices, and many others. As demonstrated in [27], these correspondences can be used to aid in the computation of Bridgeland's stability conditions.

This paper is organized as follows. In Section 2, we review the basics of path algebras, quiver representations, and gentle algebras. In Sections 3.1 and 3.2, we review the notions of the oriented flip graphs and noncrossing tree partitions that were introduced in $[\mathbf{2 1}]$.

In Sections 4 and 5, we define tiling algebras and describe all homomorphisms and extensions between indecomposable modules over tiling algebras.

In Section 6, we show that the lattice of torsion-free classes (resp., torsion classes) of $\Lambda_{T}$ ordered by inclusion (resp., reverse inclusion) is isomorphic to $\overrightarrow{F G}(T)$ (see Theorem 6.4). To obtain this result, we make use of the lattice quotient description of $\overrightarrow{F G}(T)$ from [21, Theorem 4.11] and the classification of extensions between indecomposable $\Lambda_{T}$-modules found in Section 4. In Section 7, we show that the lattice of noncrossing tree partitions of $T$ is isomorphic to the lattice of wide subcategories of $\Lambda_{T}$-mod.

In Section 8, we show that the data of a noncrossing tree partition and its Kreweras complement is equivalent to a two-term simple-minded collection of objects in the bounded derived category of $\Lambda_{T}$ (see Theorem 8.4). This theorem relies on the description of extensions between indecomposable $\Lambda_{T}$-modules found in Section 4 and on a combinatorial description of the operation of left- and right-mutation on simple-minded collections found in Section 8.1 (see Lemma 8.6). 
REMARK 1.1. An earlier version [20] of this paper was originally combined with [21]. Due to the diversity of methods and results in the original paper, we decided to divide it into two parts. In [21], we covered the combinatorial and lattice-theoretic aspects of the original paper, whereas this paper covers the representation-theoretic results.

\section{Preliminaries.}

2.1. Path algebras and quiver representations. Following [1], let $Q$ be a given quiver. We define a path of length $\ell \geq 1$ to be an expression $\alpha_{1} \alpha_{2} \cdots \alpha_{\ell}$ where $\alpha_{i} \in Q_{1}$ for all $i \in[\ell]:=\{1, \ldots, \ell\}$ and $s\left(\alpha_{i}\right)=t\left(\alpha_{i+1}\right)$ for all $i \in[\ell-1]$. We may visualize such a path in the following way:

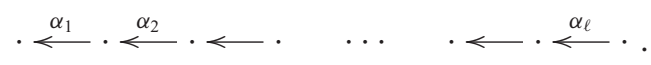

The source (resp., target) of the path $\alpha_{1} \alpha_{2} \cdots \alpha_{\ell}$ is $s\left(\alpha_{\ell}\right)$ (resp., $t\left(\alpha_{1}\right)$ ). Let $Q_{\ell}$ denote the set of all paths in $Q$ of length $\ell$. We also associate with each vertex $i \in Q_{0}$ a path of length $\ell=0$, denoted $\varepsilon_{i}$, called the lazy path at $i$. Each lazy path $\varepsilon_{i}$ satisfies $s\left(\varepsilon_{i}\right)=t\left(\varepsilon_{i}\right)=i$.

Definition 2.1. Let $Q$ be a quiver. The path algebra of $Q$, denoted $\mathbb{k} Q$, is the $\mathbb{k}$-algebra generated by all paths of length $\ell \geq 0$. Throughout this paper, we assume that $\mathbb{k}$ is algebraically closed. The multiplication of two paths $\alpha_{1} \cdots \alpha_{\ell} \in Q_{\ell}$ and $\beta_{1} \cdots \beta_{k} \in Q_{k}$ is given by the following rule:

$$
\alpha_{1} \cdots \alpha_{\ell} \cdot \beta_{1} \cdots \beta_{k}=\left\{\begin{array}{l}
\alpha_{1} \cdots \alpha_{\ell} \beta_{1} \cdots \beta_{k} \in Q_{\ell+k}: s\left(\alpha_{\ell}\right)=t\left(\beta_{1}\right) \\
0 \quad: s\left(\alpha_{\ell}\right) \neq t\left(\beta_{1}\right) .
\end{array}\right.
$$

Note that as $\mathbb{k}$-vector spaces we have

$$
\mathbb{k} Q=\bigoplus_{\ell=0}^{\infty} \mathbb{k} Q_{\ell}
$$

where $\mathbb{k} Q_{\ell}$ is the $\mathbb{k}$-vector space of all paths of length $\ell$.

In this paper, we study certain quivers $Q$ which have oriented cycles (i.e., paths $\alpha_{1} \cdots \alpha_{\ell} \in Q_{\ell}$ where $t\left(\alpha_{1}\right)=s\left(\alpha_{\ell}\right)$ ). If a quiver $Q$ possesses any oriented cycles of length $\ell \geq 1$, we see that $\mathbb{k} Q$ is infinite dimensional. In order to avoid studying infinitedimensional algebras, we will add relations to path algebras whose quivers contain oriented cycles in such a way that we obtain finite-dimensional quotients of path algebras. The relations we add are those coming from an admissible ideal $I$ of $\mathbb{k} Q$ meaning that there exists $N \geq 2$ such that

$$
\bigoplus_{\ell=N}^{\infty} \mathbb{k} Q_{\ell} \subseteq I \subseteq \bigoplus_{\ell=2}^{\infty} \mathbb{k} Q_{\ell}
$$

If $I$ is an admissible ideal of $\mathbb{k} Q$, we say that $(Q, I)$ is a bound quiver and that $\mathbb{k} Q / I$ is a bound quiver algebra.

In this paper, we study modules over a bound quiver algebra $\mathbb{k} Q / I$ by studying certain representations of $Q$ that are "compatible" with the relations coming from $I$. 
A representation $V=\left(\left(V_{i}\right)_{i \in Q_{0}},\left(\varphi_{\alpha}\right)_{\alpha \in Q_{1}}\right)$ of a quiver $Q$ is an assignment of a $\mathbb{k}$-vector space $V_{i}$ to each vertex $i$ and a $\mathbb{k}$-linear map $\varphi_{\alpha}: V_{s(\alpha)} \rightarrow V_{t(\alpha)}$ to each arrow $\alpha \in Q_{1}$. If $\rho \in \mathbb{k} Q$, it can be expressed as

$$
\rho=\sum_{i=1}^{m} c_{i} \alpha_{1}^{(i)} \cdots \alpha_{k_{i}}^{(i)}
$$

for some $c_{i} \in \mathbb{k}$ and for some $\alpha_{1}^{(i)} \cdots \alpha_{k_{i}}^{(i)} \in Q_{k_{i}}$ so when considering a representation $V$ of $Q$, we define

$$
\varphi_{\rho}:=\sum_{i=1}^{m} c_{i} \varphi_{\alpha_{1}^{(i)}} \cdots \varphi_{\alpha_{k_{i}}^{(i)}} .
$$

If we have a bound quiver $(Q, I)$, we define a representation of $Q$ bound by $I$ to be a representation of $Q$ where $\varphi_{\rho}=0$ if $\rho \in I$. We say a representation of $Q$ bound by $I$ is finite dimensional if $\operatorname{dim}_{\mathbb{k}} V_{i}<\infty$ for all $i \in Q_{0}$. It turns out that $\mathbb{k} Q / I$-mod is equivalent to the category of finite-dimensional representations of $Q$ bound by $I$. Additionally, the dimension vector of $V \in \mathbb{k} Q / I$-mod is the vector $\underline{\operatorname{dim}}(V):=\left(\operatorname{dim}_{\mathbb{k}} V_{i}\right)_{i \in Q_{0}}$ and the dimension of $V$ is defined as $\operatorname{dim}_{\mathbb{k}}(V):=\sum_{i \in Q_{0}} \operatorname{dim}_{\mathbb{k}} V_{i}$. The support of $V \in \mathbb{k} Q / I-\bmod$ is the $\operatorname{set} \operatorname{supp}(V):=$ $\left\{i \in Q_{0}: V_{i} \neq 0\right\}$.

In this paper, we focus on bound quiver algebras that are gentle algebras. A gentle algebra $\Lambda=\mathbb{k} Q / I$ is a bound quiver algebra that satisfies the following conditions:

i) each vertex of $Q$ is the starting point of at most two arrows and the ending point of at most two arrows;

ii) for each arrow $\beta \in Q_{1}$ there is at most one arrow $\alpha \in Q_{1}$ such that $\beta \alpha \notin I$, and there is at most one arrow $\gamma \in Q_{1}$ such that $\gamma \beta \notin I$;

iii) for each arrow $\beta \in Q_{1}$, there is at most one arrow $\delta \in Q_{1}$ such that $\beta \delta \in I$, and there is at most one arrow $\mu \in Q_{1}$ such that $\mu \beta \in I$;

iv) the ideal $I$ is generated by paths of length 2 .

Gentle algebras have a simple combinatorial parameterization of their indecomposable modules in terms of string modules. A string in $\Lambda$ is a sequence

$$
w=x_{1} \stackrel{\alpha_{1}}{\longleftrightarrow} x_{2} \stackrel{\alpha_{2}}{\longleftrightarrow} \cdots \stackrel{\alpha_{m}}{\longleftrightarrow} x_{m+1}
$$

where $x_{i} \in Q_{0}$ for all $i \in[m+1], \alpha_{i} \in Q_{1}$ for all $i \in[m]$, each $\alpha_{i}$ connects $x_{i}$ and $x_{i+1}$ (i.e., either $s\left(\alpha_{i}\right)=x_{i}$ and $t\left(\alpha_{i}\right)=x_{i+1}$ or $s\left(\alpha_{i}\right)=x_{i+1}$ and $t\left(\alpha_{i}\right)=x_{i}$ ), and $w$ contains no substrings of $w$ of the following forms:

i) $x \stackrel{\beta}{\longrightarrow} y \stackrel{\beta}{\longleftarrow} x$ or $x \stackrel{\beta}{\longleftarrow} y \stackrel{\beta}{\longrightarrow} x$, and

ii) $x_{i_{1}} \stackrel{\beta_{1}}{\longrightarrow} x_{i_{2}} \cdots x_{i_{s}} \stackrel{\beta_{s}}{\longrightarrow} x_{i_{s+1}}$ or $x_{i_{1}} \stackrel{\gamma_{1}}{\longleftarrow} x_{i_{2}} \cdots x_{i_{s}} \stackrel{\gamma_{s}}{\longleftarrow} x_{i_{s+1}}$ where $\beta_{s} \cdots \beta_{1}, \gamma_{1} \cdots \gamma_{s} \in I$.

In other words, $w$ is an irredundant walk in $Q$ that avoids the relations imposed by $I$. We can translate $w$ into a word $\alpha_{1}^{\epsilon_{1}} \cdots \alpha_{m}^{\epsilon_{m}}$ with $\epsilon_{i} \in\{ \pm 1\}$ for all $i \in[m]$ where $\epsilon_{i}=1$ (resp., $\epsilon_{i}=-1$ ) if $x_{i} \stackrel{\alpha_{i}}{\longrightarrow} x_{i+1}$ (resp., $x_{i} \stackrel{\alpha_{i}}{\longleftarrow} x_{i+1}$ ). Using this notation, as in [8], we identify strings with their inverses where the inverse of a string $w=\alpha_{1}^{\epsilon_{1}} \cdots \alpha_{m}^{\epsilon_{m}}$ is defined as $w^{-1}:=\alpha_{m}^{-\epsilon_{m}} \cdots \alpha_{1}^{-\epsilon_{1}}$.

Let $w=\alpha_{1}^{\epsilon_{1}} \cdots \alpha_{m}^{\epsilon_{m}}$ be a string in $\Lambda$. The string module defined by $w$ is the bound quiver representation $M(w):=\left(\left(V_{i}\right)_{i \in Q_{0}},\left(\varphi_{\alpha}\right)_{\alpha \in Q_{1}}\right)$ with 


$$
V_{i}:= \begin{cases}\bigoplus_{j: x_{j}=i} \mathbb{k} x_{j}: \text { if } i=x_{j} \text { for some } j \in[m+1] \\ 0 & : \text { otherwise }\end{cases}
$$

for each $i \in Q_{0}$ and

$$
\varphi_{\alpha}\left(x_{k}\right):= \begin{cases}x_{k-1}: & \text { if } \alpha=\alpha_{k-1} \text { and } \epsilon_{k}=-1 \\ x_{k+1}: & \text { if } \alpha=\alpha_{k} \text { and } \epsilon_{k}=1 \\ 0 \quad: \text { otherwise }\end{cases}
$$

for each $\alpha \in Q_{0}$. In the special case that $w=i$ where $i \in Q_{0}$, the string module $M(i)$ is defined as the representation of $Q$ with a one-dimensional vector space assigned to vertex $i$, the zero vector space assigned to all other vertices, and zero map assigned to all arrows. One observes that $M(w) \cong M\left(w^{-1}\right)$.

In this paper, we study a family of representation-finite gentle algebras, which we denote by $\Lambda_{T}$. It follows from [37] that the set of indecomposable modules over these algebras, denoted ind $\left(\Lambda_{T}\right.$-mod), consists of exactly the string modules $M(w)$ where $w$ is a string in $\Lambda_{T}$.

In general, gentle algebras $\Lambda$ are representation-infinite. Their indecomposable modules are parameterized by both strings and bands in $\Lambda$. We omit the definition of bands, but we remark that each band in $\Lambda$ defines an infinite family of indecomposable modules called band modules. We also omit the definition of band modules since there are no such modules belonging to ind $\left(\Lambda_{T}\right.$-mod).

EXAmPLE 2.2. Let $Q$ denote the quiver shown below. Then $\mathbb{k} Q / I=\mathbb{k} Q /\langle\alpha \beta, \beta \gamma, \gamma \alpha\rangle$ is a gentle algebra.

$$
Q=1 \stackrel{\varkappa^{2} \nwarrow_{\gamma}^{\beta}}{>} 3
$$

The algebra $\mathbb{k} Q / I$ has the following string modules:

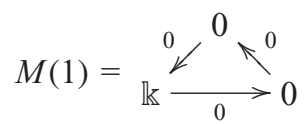

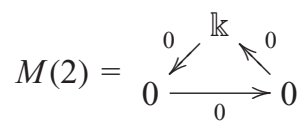

$$
\begin{aligned}
& M(3)=0 \stackrel{0}{\stackrel{\swarrow^{0}}{\longrightarrow} \mathbb{R}^{0}} \mathbb{k}
\end{aligned}
$$

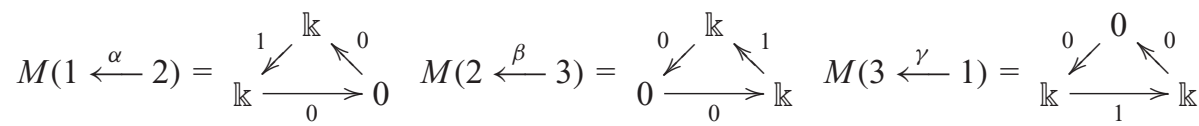

\section{Oriented flip graphs and noncrossing tree partitions.}

3.1. Oriented flip graphs. A tree is a finite, connected acyclic graph. Any tree may be embedded in a disk $D^{2}$ in such a way that a vertex is on the boundary if and only if it is a leaf. We will assume that any tree comes equipped with such an embedding. We refer to non-leaf vertices of a tree as interior vertices, and, by convention, any interior vertex has degree at least 3 . The embedding that accompanies $T$ also endows each interior vertex 
with a cyclic ordering. In addition, we say two trees $T$ and $T^{\prime}$ are equivalent if there is an isotopy between the spaces $D^{2} \backslash T$ and $D^{2} \backslash T^{\prime}$.

A tree $T$ embedded in $D^{2}$ determines a collection of two-dimensional regions in $D^{2}$ that we will refer to as faces. A corner of a tree is a pair $(v, F)$ consisting of an interior vertex $v$ and a two-dimensional face $F$ containing $v$. We let $\operatorname{Cor}(T)$ denote the set of corners of $T$.

An acyclic path (or chordless path) supported by a tree $T$ is a sequence $\left(v_{0}, \ldots, v_{t}\right)$ of pairwise distinct vertices of $T$ such that $v_{i}$ and $v_{j}$ are adjacent if and only if $|i-j|=1$. We typically identify acyclic paths with their underlying vertex sets; that is, we do not distinguish between acyclic paths of the form $\left(v_{0}, \ldots, v_{t}\right)$ and $\left(v_{t}, \ldots, v_{0}\right)$. We will refer to $v_{0}$ and $v_{t}$ as the end points of the acyclic path $\left(v_{0}, \ldots, v_{t}\right)$. Note that an acyclic path is determined by its end points, and thus we can write $\left[v_{0}, v_{t}\right]=\left(v_{0}, \ldots, v_{t}\right)$. As an acyclic path $\left(v_{0}, \ldots, v_{t}\right)$ defines a subgraph of $T$ (namely, the induced subgraph on the vertices $\left.v_{0}, \ldots, v_{t}\right)$, it makes sense to refer to an edge of $\left(v_{0}, \ldots, v_{t}\right)$. Additionally, if $\left(v_{0}, \ldots, v_{t}\right)$ and $\left(v_{t}, \ldots, v_{t+s}\right)$ are acyclic paths that agree only at $v_{t}$ and where $\left[v_{0}, v_{t+s}\right]$ is an acyclic path, we define their composition as $\left[v_{0}, v_{t}\right] \circ\left[v_{t}, v_{t+s}\right]:=\left[v_{0}, v_{t+s}\right]$.

A segment $s=\left(v_{0}, \ldots, v_{t}\right)$ is an acyclic path consisting of at least two vertices where any two edges $\left(v_{i-1}, v_{i}\right)$ and $\left(v_{i}, v_{i+1}\right)$ are incident to a common face and whose end points are not leaves. Observe that interior vertices of $T$ are not segments. If the composition $s \circ t$ of two segments $s$ and $t$ is a segment, we say that $s$ and $t$ are composable.

Let $\operatorname{Seg}(T)$ be the set of segments supported by a tree $T$. Given $X \subseteq \operatorname{Seg}(T)$, we say $X$ is closed if for any pair of composable segments $s, t \in X$ one has $s \circ t \in X$. If $X$ is any subset of $\operatorname{Seg}(T)$, its closure, denoted $\bar{X}$, is the smallest closed set containing $X$. Say $X$ is biclosed if $X$ and $\operatorname{Seg}(T) \backslash X$ are both closed. At times, we will also say $X$ is co-closed if $\operatorname{Seg}(T) \backslash X$ is closed.

Define $\operatorname{Bic}(T)$ to be the collection of biclosed subsets of $\operatorname{Seg}(T)$, partially ordered by inclusion. The poset $\operatorname{Bic}(T)$ is a lattice with many nice properties. In particular, for any biclosed sets $B_{1}, B_{2} \in \operatorname{Bic}(T)$, we have that $B_{1} \vee B_{2}=\overline{B_{1} \cup B_{2}}$. We refer the reader to [21] for more information on the lattice structure of biclosed sets.

Let $s=\left(v_{0}, \ldots, v_{l}\right)$ be a segment, and orient the segment from $v_{0}$ to $v_{l}$. Let $C_{s}$ be the set of segments $\left(v_{i}, \ldots, v_{j}\right)$ such that

- if $i>0$ then $s$ turns right at $v_{i}$, and

- if $j<l$ then $s$ turns left at $v_{j}$.

We note that $s$ is always in $C_{s}$ since the above conditions are vacuously true. Furthermore if $t \in C_{s}$, then $C_{t} \subseteq C_{s}$. Let $\pi_{\downarrow}: \operatorname{Bic}(T) \rightarrow \operatorname{Bic}(T)$ be the function such that for any $X \in$ $\operatorname{Bic}(T)$,

$$
\pi_{\downarrow}(X):=\left\{s \in X: C_{s} \subseteq X\right\} .
$$

It follows from [21, Lemma 4.4] that $\pi_{\downarrow}(X)$ is actually biclosed. Define the oriented flip graph of $T$, denoted $\overrightarrow{F G}(T)$, to be the partially ordered set $\pi_{\downarrow}(\operatorname{Bic}(T))$. The name for this poset comes from the fact that its elements can be identified with a family of partial triangulations of a disk whose covering relations correspond to flips. This identification is not obvious from the definition we give here, but it was proved in [21, Theorem 4.14]. This theorem identifies the oriented flip graph as both a sublattice and a quotient lattice of $\operatorname{Bic}(T)$. As such, it is also a lattice.

EXAMPLE 3.1. Let $T$ denote the tree appearing in Figure 1. Observe that $\operatorname{Seg}(T)=$ $\{s, t, s \circ t\}$. In Figure 2, we show the lattice of biclosed sets of $T$ and the oriented flip graph 


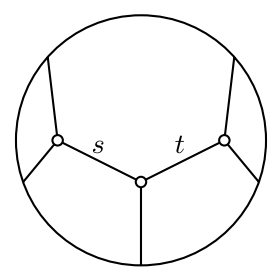

Figure 1. An example of a tree.
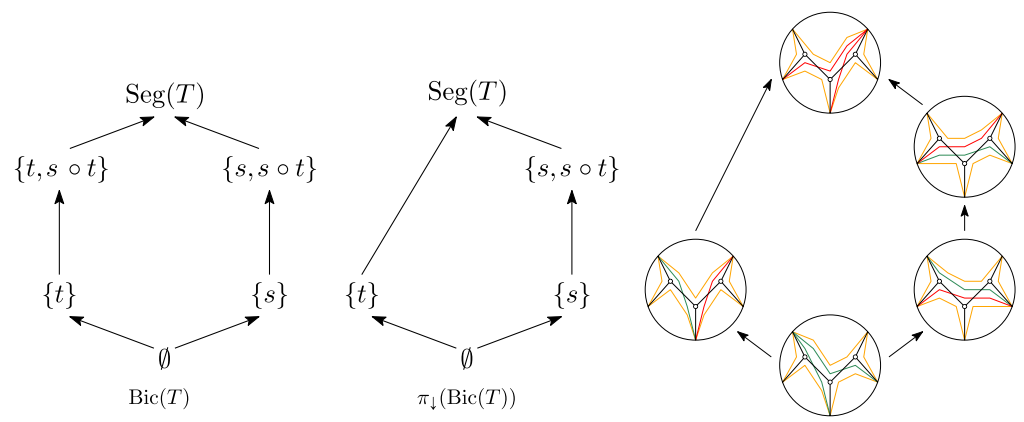

Figure 2. (Color online) The lattice of biclosed sets of segments and the oriented flip graph of the tree from Figure 1.

of $T$ defined as $\pi_{\downarrow}(\operatorname{Bic}(T))$. On the right, we also show the oriented flip graph, as defined in [21], whose elements may be identified with partial triangulations of a disk.

The reader may find additional examples of oriented flip graphs in [21].

3.2. Noncrossing tree partitions. Let $V^{o}$ denote the set of interior vertices of $T$. Fix a small $\epsilon>0$ such that the $\epsilon$-ball centered at any interior vertex of $T$ is contained in $D^{2}$, and no two such $\epsilon$-balls intersect. For each corner $(v, F)$, we fix a point $z(v, F)$ in the interior of $F$ of distance $\epsilon$ from $v$. Let

$$
T_{\epsilon}:=T \cup \bigcup_{v \in V^{o}}\left\{x \in D^{2}:|x-v|<\epsilon\right\} .
$$

In other words, $T_{\epsilon}$ is the embedded tree $T$ plus the open $\epsilon$-ball around each interior vertex. Similarly, given $[u, v] \in \operatorname{Seg}(T)$, let $[u, v]_{\epsilon}$ denote the subset of $T_{\epsilon}$ that supports $[u, v]$ and the $\epsilon$ balls around $u$ and around $v$.

It will be convenient to represent segments as certain curves in the disk as follows. A flag is a triple $(v, e, F)$ of a vertex $v$ incident to an edge $e$, which is incident to a face $F$. Orienting $e$ away from $v$, we say a flag is green if $F$ is left of $e$. Otherwise, the flag is red. Let $(u, e, F),\left(v, e^{\prime}, G\right)$ be two green flags such that $[u, v]$ is a segment containing the edges $e, e^{\prime}$ as in Figure 3. A green admissible curve $\gamma:[0,1] \rightarrow D^{2}$ for $[u, v]$ is a simple curve for which $\gamma(0)=z(u, F), \gamma(1)=z(v, G)$ and $\gamma([0,1]) \subseteq D^{2} \backslash\left(T_{\epsilon} \backslash[u, v]_{\epsilon}\right)$. Similarly, if $\left(u, e, F^{\prime}\right)$ and $\left(v, e^{\prime}, G^{\prime}\right)$ are red flags, then a red admissible curve is defined the same way, with $\gamma(0)=z\left(u, F^{\prime}\right), \gamma(1)=z\left(v, G^{\prime}\right)$. We say a segment is green if it is represented by a green admissible curve. Similarly, a segment is red if it is represented by a red admissible curve. We may also refer to an admissible curve for a segment without specifying a color. Such a curve may be either green or red.

If a colored segment $s$ is represented by a curve with end points $z(u, F)$ and $z(v, G)$, we say that $(u, F)$ and $(v, G)$ are the end points of $s$. We refer to corners or vertices as the 

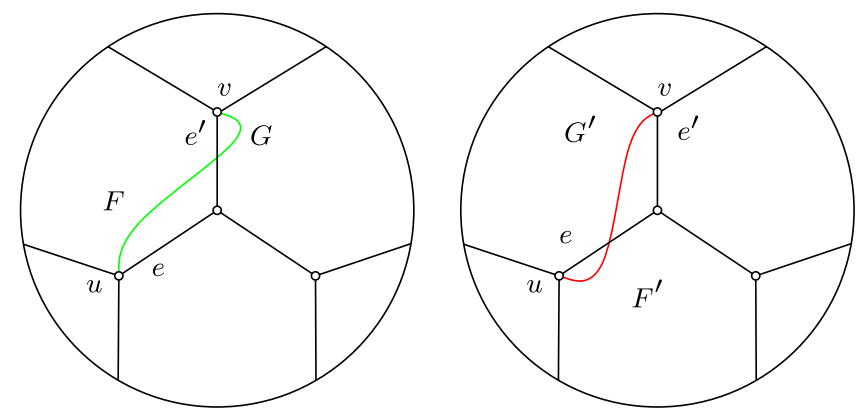

Figure 3. (Color online) A green admissible curve and a red admissible curve for the segment $[u, v]$.
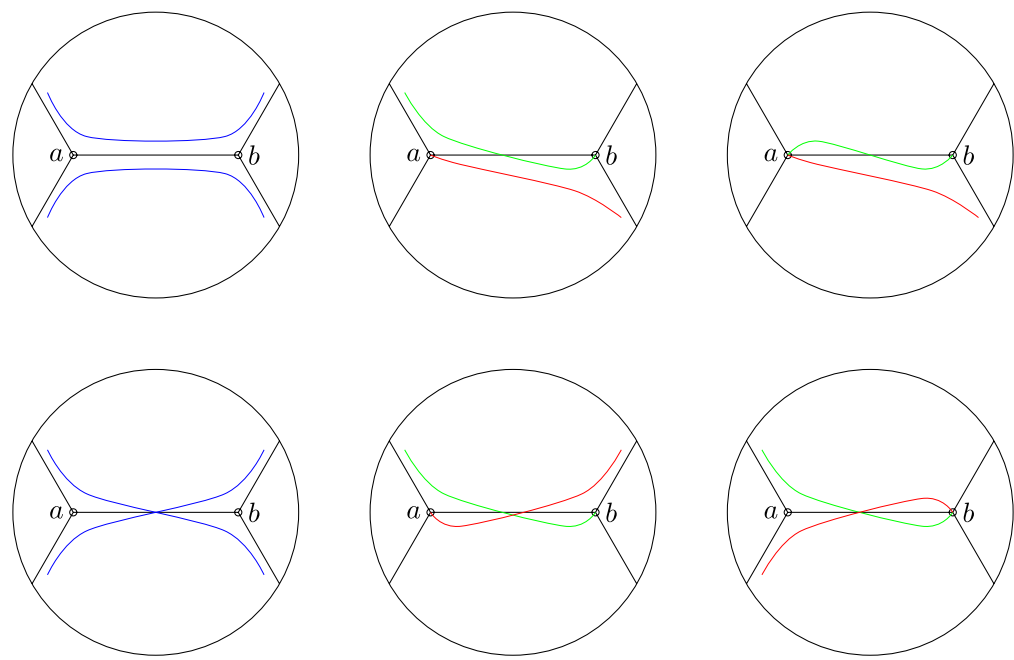

Figure 4. (Color online) Several examples of crossing and noncrossing admissible curves representing segments supported by the tree.

end points of a segment at different parts of this paper. The distinction should be clear from context.

Two colored segments are noncrossing if they admit admissible curves that do not intersect. Otherwise, they are crossing. We remark that if two curves share an end point $z(u, F)$, then they are considered to be crossing. To determine whether two colored segments $s, t$ cross, one must check whether the end points of $t$ lie in different connected components of $\left(D^{2} \backslash\left(T_{\epsilon} \backslash t_{\epsilon}\right)\right) \backslash \gamma$ for some admissible curve $\gamma$ for $s$. We will find it convenient to distinguish several cases of crossing as in the following lemma. The three cases correspond to the three columns of Figure 4.

Lemma 3.2. Let $\gamma$ and $\gamma^{\prime}$ be two (green or red) admissible curves corresponding to segments $s$ and $s^{\prime}$ that meet along a common segment $t$. Let $t=[a, b]$ and orient $\gamma$ and $\gamma^{\prime}$ from a to $b$. Assume that $\gamma$ and $\gamma^{\prime}$ do not share an end point. Then $\gamma$ and $\gamma^{\prime}$ are noncrossing if and only if one of the following holds:

(1) $s$ (or $\left.s^{\prime}\right)$ does not share an end point with $t$, and $\gamma$ turns left (or right) at both end points of $t$;

(2) $\gamma$ starts at a and turns left (resp., right) at b, and $\gamma^{\prime}$ ends at b and turns right (resp., left) at a; 


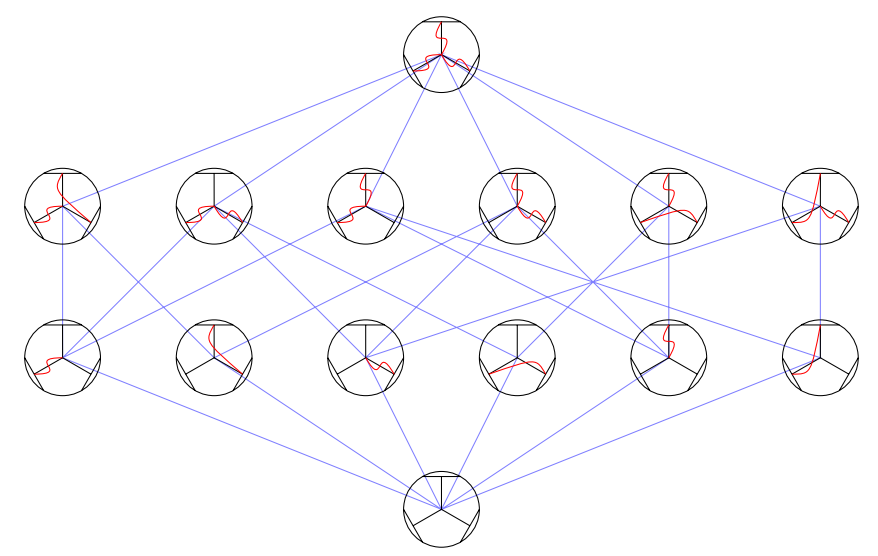

Figure 5. (Color online) A lattice of noncrossing tree partitions.

(3) $\gamma$ and $\gamma^{\prime}$ both start at a (resp., both end at b) where $\gamma$ leaves a (resp., b) to the left, and $\gamma$ turns left at $b$ (resp., a) or $\gamma^{\prime}$ turns right at $b$ (resp., $\left.a\right)$.

If $\gamma$ and $\gamma^{\prime}$ are both green admissible or both red admissible, then the third case does not occur.

For $B \subseteq V^{o}$, let $\operatorname{Seg}(B)$ be the set of inclusion-minimal segments whose end points lie in $B$. That is, there do not exist distinct segments $s, t \in \operatorname{Seg}(B)$ where every vertex of $t$ appears in $s$. We say $B$ is segment-connected if for any two elements $u, v$ of $B$, there exists a sequence $u=u_{0}, \ldots, u_{N}=v$ of elements of $B$ such that $\left[u_{i-1}, u_{i}\right] \in \operatorname{Seg}(B)$ for all $i$. If $\mathbf{B}=\left\{B_{1}, \ldots, B_{l}\right\}$ is a partition of $V^{o}$, we let $\operatorname{Seg}(\mathbf{B})=\bigcup_{i=1}^{l} \operatorname{Seg}\left(B_{i}\right)$. We let $\operatorname{Seg}_{g}(\mathbf{B})$ (resp., $\left.\operatorname{Seg}_{r}(\mathbf{B})\right)$ denote the same set of segments, all colored green (resp., red).

A noncrossing tree partition $\mathbf{B}$ is a set partition of $V^{o}$ such that any two segments of $\mathrm{Seg}_{r}(\mathbf{B})$ are noncrossing and each block of $\mathbf{B}$ is segment-connected. Note that we intentionally define noncrossing tree partitions using only red segments. Let $\operatorname{NCP}(T)$ be the poset of noncrossing tree partitions of $T$, ordered by refinement. By [21, Theorem 5.13], the poset $\operatorname{NCP}(T)$ is a lattice. In fact, in [21, Theorem 5.15] we show that $\operatorname{NCP}(T)$ is isomorphic to the "lattice-theoretic" shard intersection order (see [22]) of $\overrightarrow{F G}(T)$, denoted $\Psi(\overrightarrow{F G}(T))$, via the isomorphism $\mathbf{B} \mapsto \overline{\operatorname{Seg}(\mathbf{B})}$. Here $\overline{\operatorname{Seg}(\mathbf{B})}$ is the smallest closed set of segments containing $\operatorname{Seg}(\mathbf{B})$.

We give an example of $\operatorname{NCP}(T)$ in Figure 5 where $T$ is the tree appearing in Figure 3. We remark that this lattice of noncrossing tree partitions is not isomorphic to the lattice of noncrossing set partitions of $\{1,2,3,4\}$.

By [21, Corollary 5.12], there is a distinguished bijection $\mathrm{Kr}: \operatorname{NCP}(T) \rightarrow \operatorname{NCP}(T)$. We call $\operatorname{Kr}(\mathbf{B})$ the Kreweras complement of $\mathbf{B}$. The noncrossing tree partition $\operatorname{Kr}(\mathbf{B})$ is characterized by the property that there exist red admissible curves for $\operatorname{Seg}(\mathbf{B})$ and green admissible curves for $\operatorname{Seg}(\operatorname{Kr}(\mathbf{B}))$ such that when one superimposes these curves on $T$, one obtains a noncrossing tree whose vertex set is $V^{o}$. We show an example of a noncrossing tree partition and its Kreweras complement in Figure 7.

4. Trees and their tiling algebras. Let $T$ be a tree embedded in $D^{2}$. Then $T$ defines a bound quiver, denoted $\left(Q_{T}, I_{T}\right)$, as follows. Let $Q_{T}$ be the quiver whose vertices are in 

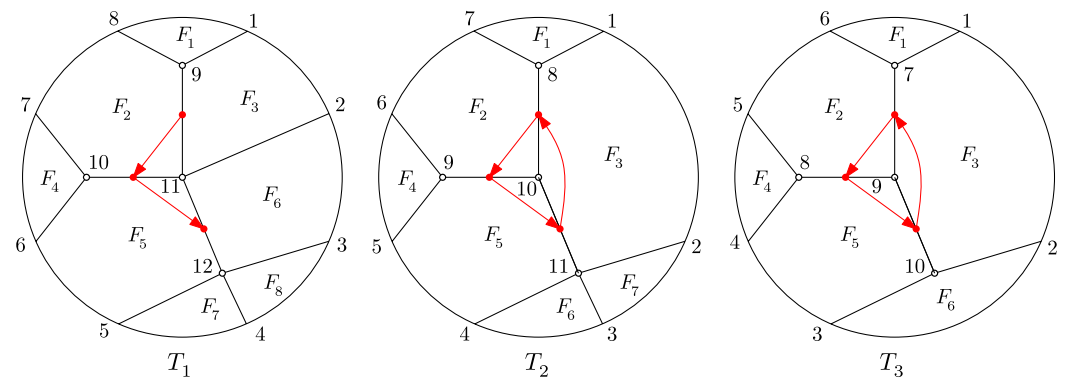

Figure 6. (Color online) Three examples of the quiver defined by a tree.

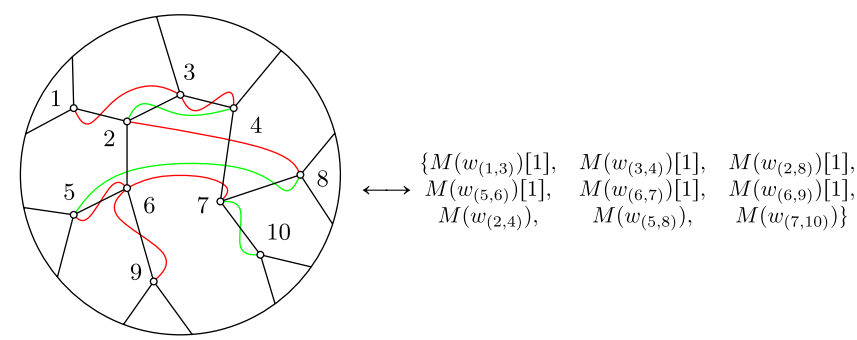

Figure 7. (Color online) The noncrossing tree partition $\mathbf{B}=(\{1,3,4\},\{2,8\},\{5,6,7,9\},\{10\})$ with its Kreweras complement $\operatorname{Kr}(\mathbf{B})=(\{1\},\{2,4\},\{3\},\{5,8\},\{6\},\{7,10\},\{9\})$ and its corresponding simple-minded collection via the map $\theta$ in Theorem 8.4. Here $w_{(i, j)}$ denotes the string corresponding to the segment of $T$ connecting $i$ and $j$.

bijection with the edges of $T$ that contain no leaves and whose arrows are exactly those of the form $e_{1} \stackrel{\alpha}{\longrightarrow} e_{2}$ satisfying:

i) $e_{1}$ and $e_{2}$ define a corner of $T$,

ii) $e_{2}$ is counterclockwise from $e_{1}$.

The admissible ideal $I_{T}$ is, by definition, generated by the relations $\alpha \beta$ where $\alpha: e_{2} \longrightarrow e_{3}$ defines the corner $(v, F)$ and $\beta: e_{1} \longrightarrow e_{2}$ defines the corner $(v, G)$. We define the tiling algebra of $T$ to be $\Lambda_{T}:=\mathbb{k} Q_{T} / I_{T}$. We remark that the term tiling algebra first appeared in [12] where a tiling algebra is defined by a partial triangulation of a polygon.

EXAMPLE 4.1. In Figure 6, we show three trees. The tree $T_{1}$ determines the quiver $Q_{T_{1}}=1 \stackrel{\beta}{\longrightarrow} 2 \stackrel{\alpha}{\longrightarrow} 3$. The tiling algebra of $T_{1}$ is $\Lambda_{T_{1}}=\mathbb{k} Q_{T_{1}} / I_{T_{1}}$ where $I_{T_{1}}=\langle\alpha \beta\rangle$. Also note that $Q_{T_{2}} \cong Q_{T_{3}} \cong Q$ and $\Lambda_{T_{2}} \cong \Lambda_{T_{3}} \cong \Lambda$ where $Q$ is the quiver from Example 2.2 and $\Lambda$ is the algebra from Example 2.2.

Proposition 4.2. The algebra $\Lambda_{T}$ is a gentle algebra. Furthermore, the algebra $\Lambda_{T}$ is representation-finite and its indecomposables are exactly the string modules.

Proof. The first assertion follows from [12, Proposition 3.2]. To prove the second assertion, it is enough to observe that any string $w$ in $\Lambda_{T}$ can be regarded as a full, connected subquiver of $Q_{T}$ with at most one arrow from any cycle in $Q_{T}$. This implies that there are no cyclic strings in $\Lambda_{T}$ and therefore no bands in $\Lambda_{T}$.

COROLLARY 4.3. The following hold for the tiling algebra $\Lambda_{T}$.

(1) Assume $M(w):=\left(\left(V_{i}\right)_{i \in Q_{0}},\left(\varphi_{\alpha}\right)_{\alpha \in Q_{1}}\right)$ is a string module of $\Lambda_{T}$. Then $\operatorname{dim}_{\mathbb{k}}\left(V_{i}\right)=1$ if $i \in \operatorname{supp}(M(w))$ and $\operatorname{dim}_{\mathbb{k}}\left(V_{i}\right)=0$ otherwise. 
(2) The map ind $\left(\Lambda_{T}-\bmod \right) \longrightarrow \operatorname{Seg}(T)$ defined by

$$
M(w) \longmapsto s_{w}:=\left(v_{0}, \ldots, v_{t}\right)
$$

where each $v_{i}$ is a vertex of $T$ belonging to some $e_{j} \in \operatorname{supp}(M(w))$ and where each pair $v_{i}$ and $v_{i+1}$ belongs to a common $e_{j} \in \operatorname{supp}(M(w))$ is a bijection.

Proof. Assertion (1) follows from the proof of Proposition 4.2.

To prove assertion (2), note that any string module $M(w) \in \operatorname{ind}\left(\Lambda_{T}\right.$-mod) can be regarded as a subpath of $Q_{T}$ each of whose nonzero 2-paths does not belong to $I_{T}$. With this identification, we observe that $M(w)$ is equivalent to a sequence of distinct interior vertices $\left(v_{0}, \ldots, v_{t}\right)$ of $Q_{T}$ with the property that any two edges $\left(v_{i-1}, v_{i}\right)$ and $\left(v_{i}, v_{i+1}\right)$ are contained in a common face of $T$. Thus, the given map is a bijection.

REMARK 4.4. Corollary 4.3 implies that every indecomposable module of $\Lambda_{T}$ is completely determined by its support. We tacitly make use of this fact from this point onwards.

We now present a description of the spaces of extensions between indecomposable $\Lambda_{T}$ modules. These results generalize the description of extensions between indecomposable modules over the Jacobian algebra arising from a triangulation of an unpunctured surface found in $[\mathbf{8}]$ in the case where the surface is a disk. We note that in [8] the authors consider triangulations of other surfaces, but they do not consider partial triangulations of these surfaces. The proofs of the following results depend on some technical lemmas presented in Section 5.

The following results (Propositions 4.5 and 4.6 and Theorems 4.7 and 4.8) were new when we originally announced them in [20]. Since then, the space of extensions between indecomposable modules over a general gentle algebra has been described in $[\mathbf{9}, \mathbf{1 0}]$. Although the results therein imply ours, we include the proofs of our results as they appeared in [20].

Proposition 4.5. Let $M(u), M(v) \in \operatorname{ind}\left(\Lambda_{T}\right.$-mod) where $s_{u}$ and $s_{v}$ do not have any common vertices. Then $\operatorname{Ext}_{\Lambda_{T}}^{1}(M(v), M(u))=0$.

Proof. Since $s_{u}$ and $s_{v}$ have no common vertices, there is no arrow $\alpha \in\left(Q_{T}\right)_{1}$ such that $u \stackrel{\alpha}{\leftarrow} v$ is a string in $\Lambda_{T}$. By Lemma 5.6, it follows that $\operatorname{Ext}_{\Lambda_{T}}^{1}(M(v), M(u))=0$.

Proposition 4.6. Let $M(u), M(v) \in \operatorname{ind}\left(\Lambda_{T}-\right.$ mod $)$ where $s_{u}$ and $s_{v}$ either share an end point and agree along a segment or they have a common vertex that is an end point of at most one of $s_{u}$ and $s_{v}$. Then $\operatorname{Ext}_{\Lambda_{T}}^{1}(M(v), M(u))=0$.

Proof. Let $0 \rightarrow M(u) \stackrel{f}{\rightarrow} X \stackrel{g}{\rightarrow} M(v) \rightarrow 0$ be an extension. By Lemma $5.9 i), X$ has at least two summands $M(y)$ and $M(z)$ for some nonempty strings $y$ and $z$ in $\Lambda_{T}$. By Lemma $5.9 \mathrm{ii}$ ), without loss of generality, we have that $M(y)=M(u)$ and $M(z)=M(v)$ so the given sequence is split.

THEOREM 4.7. Let $M(u), M(v) \in \operatorname{ind}\left(\Lambda_{T}-\bmod \right)$ where $s_{u}$ and $s_{v}$ agree only at an end point. Then there is a nonsplit extension $\xi=0 \rightarrow M(u) \rightarrow M(u \stackrel{\alpha}{\leftarrow} v) \rightarrow M(v) \rightarrow 0$ if and only if there exists an arrow $\alpha \in\left(Q_{T}\right)_{1}$ such that $u \stackrel{\alpha}{\leftarrow} v$ is a string in $\Lambda_{T}$. In this case, $\xi$ is the unique nonsplit extension of $M(v)$ by $M(u)$.

Proof. Assume that there exists an arrow $\alpha \in\left(Q_{T}\right)_{1}$ such that $u \stackrel{\alpha}{\leftarrow} v$ is a string in $\Lambda_{T}$. Thus, $M(u \stackrel{\alpha}{\leftarrow} v)$ is a string module and so $\xi$ is a nonsplit extension. 
Assume that there does not exist an arrow $\alpha \in\left(Q_{T}\right)_{1}$ such that $u \stackrel{\alpha}{\leftarrow} v$ is a string in $\Lambda_{T}$. Let $0 \rightarrow M(u) \stackrel{f}{\rightarrow} X \stackrel{g}{\rightarrow} M(v) \rightarrow 0$ be an extension. Lemma 5.6 implies that $X=M(u) \oplus$ $M(v)$ so all such extensions are split.

The last assertion follows from the fact that $\operatorname{dim}_{\mathbb{k}} \operatorname{Ext}_{\Lambda_{T}}^{1}(M(v), M(u))=1$ by Lemma 5.5.

THEOREM 4.8. Let $M(u), M(v) \in \operatorname{ind}\left(\Lambda_{T}\right.$-mod $)$ and suppose that $\operatorname{supp}(M(u)) \cap$ $\operatorname{supp}(M(v)) \neq \varnothing$. Now let $w$ denote the unique maximal string supported on $\operatorname{supp}(M(u)) \cap$ $\operatorname{supp}(M(v))$. Furthermore, assume that the segments $s_{u}$ and $s_{v}$ do not have any common end points. Write $u=u^{(1)} \leftrightarrow w \leftrightarrow u^{(2)}$ and $v=v^{(1)} \leftrightarrow w \leftrightarrow v^{(2)}$ for some strings $u^{(1)}, u^{(2)}, v^{(1)}$, and $v^{(2)}$ in $\Lambda_{T}$, some of which may be empty. Then $\operatorname{Ext}_{\Lambda_{T}}^{1}(M(v), M(u)) \neq 0$ if and only if $u=u^{(1)} \leftarrow w \rightarrow u^{(2)}$ and $v=v^{(1)} \rightarrow w \leftarrow v^{(2)}$. Additionally, in this case,

$$
0 \rightarrow M(u) \rightarrow M\left(u^{(1)} \leftarrow w \leftarrow v^{(2)}\right) \oplus M\left(v^{(1)} \rightarrow w \rightarrow u^{(2)}\right) \rightarrow M(v) \rightarrow 0
$$

is the unique nonsplit extension of $M(v)$ by $M(u)$.

Proof. Assume that $u=u^{(1)} \leftarrow w \rightarrow u^{(2)}$ and $v=v^{(1)} \rightarrow w \leftarrow v^{(2)}$ for some strings $u^{(1)}, u^{(2)}, v^{(1)}$, and $v^{(2)}$ in $\Lambda_{T}$, not all of which are empty. Note that the segments $s_{u}$ and $s_{v}$ have no common end points. This means that $M\left(u^{(1)} \leftarrow w \leftarrow v^{(2)}\right)$ is not isomorphic to $M(u)$ or $M(v)$ and the same is true for $M\left(v^{(1)} \rightarrow w \rightarrow u^{(2)}\right)$. Thus,

$$
0 \rightarrow M(u) \rightarrow M\left(u^{(1)} \leftarrow w \leftarrow v^{(2)}\right) \oplus M\left(v^{(1)} \rightarrow w \rightarrow u^{(2)}\right) \rightarrow M(v) \rightarrow 0
$$

is a nonsplit extension. This implies that $\operatorname{Ext}_{\Lambda_{T}}^{1}(M(v), M(u)) \neq 0$.

Conversely, assume that $\operatorname{Ext}_{\Lambda_{T}}^{1}(M(v), M(u)) \neq 0$. Let $0 \rightarrow M(u) \stackrel{f}{\rightarrow} X \stackrel{g}{\rightarrow} M(v) \rightarrow 0$ be a nonsplit extension and let $X=\oplus_{i=1}^{k} X_{i}$ be a direct sum decomposition of $X$ into indecomposables. By Corollary 5.8, we have that $X=M\left(u^{(1)} \leftrightarrow w \leftrightarrow v^{(2)}\right) \oplus M\left(v^{(1)} \leftrightarrow\right.$ $\left.w \leftrightarrow u^{(2)}\right)$. Since the given sequence is exact, we must have that $\left(u^{(1)} \leftrightarrow w \leftrightarrow v^{(2)}\right)=$ $\left(u^{(1)} \leftarrow w \leftarrow v^{(2)}\right)$ and $\left(v^{(1)} \leftrightarrow w \leftrightarrow u^{(2)}\right)=\left(v^{(1)} \rightarrow w \rightarrow u^{(2)}\right)$. Thus, $u=u^{(1)} \leftarrow w \rightarrow u^{(2)}$ and $v=v^{(1)} \rightarrow w \leftarrow v^{(2)}$.

The last assertion follows from the fact that $\operatorname{dim}_{\mathbb{k}} \operatorname{Ext}_{\Lambda_{T}}^{1}(M(v), M(u))=1$ by Lemma 5.5.

5. Homomorphisms and extensions between string modules. In this section, we present the technical facts required to prove Propositions 4.5 and 4.6 and Theorems 4.7 and 4.8. We prove Lemma 5.1, which is used in the statement of Theorem 4.8, Lemma 5.7, and Corollary 5.8. We omit the proofs of Lemma 5.2, 5.3, and 5.5 as they are nearly identical to that of [19, Lemma 9.2], [19, Lemma 9.3], and [19, Lemma 9.4], respectively.

LEMMA 5.1. Let $M(u), M(v) \in \operatorname{ind}\left(\Lambda_{T}\right.$-mod) with $\operatorname{supp}(M(u)) \cap \operatorname{supp}(M(v)) \neq \emptyset$. Then $w=x_{1} \leftrightarrow x_{2} \cdots x_{k-1} \leftrightarrow x_{k}$ where $\operatorname{supp}(M(u)) \cap \operatorname{supp}(M(v))=\left\{x_{i}\right\}_{i \in[k]}$ is a string in $\Lambda_{T}$. Furthermore, $w$ is the unique maximal string along which $u$ and $v$ agree.

Proof. Any string in $\Lambda_{T}$ includes at most two vertices from any oriented cycle in $Q_{T}$. Thus, a string $u=y_{1} \leftrightarrow y_{2} \cdots y_{s-1} \leftrightarrow y_{s}$ is the shortest path connecting $y_{1}$ and $y_{s}$ in the underlying graph of $Q_{T}$. This implies that for any $y_{i}$ and $y_{j}$ appearing in $u$, the string $y_{i} \leftrightarrow y_{i+1} \cdots y_{j-1} \leftrightarrow y_{j}$ is the shortest path connecting $y_{i}$ and $y_{j}$ in the underlying graph of $Q_{T}$. Therefore if $\operatorname{supp}(M(u)) \cap \operatorname{supp}(M(v)) \neq \emptyset$, then $w=x_{1} \leftrightarrow x_{2} \cdots x_{k-1} \leftrightarrow x_{k}$ where 
$\operatorname{supp}(M(u)) \cap \operatorname{supp}(M(v))=\left\{x_{i}\right\}_{i \in[k]}$ is a string in $\Lambda_{T}$. Clearly, $w$ is the unique maximal string along which $u$ and $v$ agree.

Lemma 5.2. Let $M(u), M(v) \in \operatorname{ind}\left(\Lambda_{T}-\right.$ mod $)$. If $M(u) \hookrightarrow M(v)$ or $M(u) \rightarrow M(v)$, then

$$
\operatorname{dim}_{\mathbb{k}} \operatorname{Hom}_{\Lambda_{T}}(M(u), M(v))=1
$$

Lemma 5.3. Let $M(u), M(v) \in \operatorname{ind}\left(\Lambda_{T}-\bmod \right)$. Then $\operatorname{dim}_{\mathbb{k}} \operatorname{Hom}_{\Lambda_{T}}(M(u), M(v)) \leq 1$. Additionally, assume $\operatorname{Hom}_{\Lambda_{T}}(M(u), M(v)) \neq 0$, but $M(u)$ is not a submodule of $M(v)$ and $M(u)$ does not surject onto $M(v)$. Then there exists a string $w$ in $\Lambda_{T}$ distinct from both $u$ and $v$ such that $M(u) \rightarrow M(w) \hookrightarrow M(v)$.

Lemma 5.4. Assume $s_{u}$ and $s_{v}$ agree at an end point. Then $s_{u}$ and $s_{v}$ agree along a segment $s_{w}$ if and only if either $\operatorname{Hom}_{\Lambda_{T}}(M(u), M(v)) \neq 0$ or $\operatorname{Hom}_{\Lambda_{T}}(M(v), M(u)) \neq 0$.

Proof. Assume $s_{u}$ and $s_{v}$ agree along a segment $s_{w}$. By Lemma 5.1, assume that $s_{w}$ is the unique largest segment along which $s_{u}$ and $s_{v}$ agree. We have that either $u=u^{(1)} \leftarrow w$ and $v=v^{(1)} \rightarrow w$ or $u=u^{(1)} \rightarrow w$ and $v=v^{(1)} \leftarrow w$. In the former case, $\operatorname{Hom}_{\Lambda_{T}}(M(u), M(v)) \neq 0$. In the latter case, $\operatorname{Hom}_{\Lambda_{T}}(M(v), M(u)) \neq 0$.

The converse statement is obvious.

Lemma 5.5. Let $M(u), M(v) \in \operatorname{ind}\left(\Lambda_{T}\right.$-mod $)$. Then $\operatorname{dim}_{\mathbb{k}} \operatorname{Ext}_{\Lambda_{T}}^{1}(M(u), M(v)) \leq 1$.

Next, we present four results, each of which is crucial to classifying extensions between indecomposable $\Lambda_{T}$-modules. Lemma 5.6 is used in the proof of Proposition 4.5 and Theorem 4.7. Corollary 5.8, which is used in the proof of Theorem 4.8, follows from Lemma 5.7. Lemma 5.7 establishes several restrictions on which indecomposable $\Lambda_{T}$-modules can appear as middle terms of a nonsplit extension between two indecomposables whose corresponding segments agree along a segment, but have no shared end points. Lastly, Lemma 5.9 is used in the proof of Proposition 4.6.

LEMMA 5.6. Let $0 \rightarrow M(u) \stackrel{f}{\rightarrow} X \stackrel{g}{\rightarrow} M(v) \rightarrow 0$ be an extension where $\operatorname{supp}(M(u)) \cap$ $\operatorname{supp}(M(v))=\emptyset$. Assume that there does not exist an arrow $\alpha \in\left(Q_{T}\right)_{1}$ such that $u \stackrel{\alpha}{\leftarrow} v$ is a string in $\Lambda_{T}$ and let $X=\oplus_{i=1}^{k} X_{i}$ be a direct sum decomposition of $X$ into indecomposables (i.e., $X_{i} \in \operatorname{ind}\left(\Lambda_{T}\right.$-mod) for each $\left.i \in[k]\right)$. Then none of the modules $X_{i}$ have any of the following properties:

i) $\operatorname{supp}\left(X_{i}\right) \cap \operatorname{supp}(M(u)) \neq \varnothing$ and $\operatorname{supp}\left(X_{i}\right) \cap \operatorname{supp}(M(v)) \neq \emptyset$

ii) $\operatorname{supp}\left(X_{i}\right) \subsetneq \operatorname{supp}(M(u))$

iii) $\operatorname{supp}\left(X_{i}\right) \subsetneq \operatorname{supp}(M(v))$.

Proof. Suppose some $X_{i}$ satisfies $\left.i\right)$. Then we can write $X_{i}=M(w), u=u^{\prime} \leftrightarrow w^{\prime}$, and $v=w^{\prime \prime} \leftrightarrow v^{\prime \prime}$ where $w=w^{\prime} \leftrightarrow w^{\prime \prime}$ is a string in $\Lambda_{T}$. By assumption, $w=w^{\prime} \leftrightarrow w^{\prime \prime}=$ $w^{\prime} \stackrel{\beta}{\rightarrow} w^{\prime \prime}$. Observe that the direction of $\beta$ implies that $\operatorname{Hom}_{\Lambda_{T}}(M(u), M(w))=0$ and $\operatorname{Hom}_{\Lambda_{T}}(M(w), M(v))=0 . \operatorname{Since} \operatorname{supp}(M(u)) \cap \operatorname{supp}(M(v))=\emptyset,\left\{\operatorname{supp}\left(X_{i}\right)\right\}_{i=1}^{k}$ is a set partition of the set $\operatorname{supp}(X)$. Thus, we have that $M(w) \cap \operatorname{im}(f)=0$, but $M(w) \subseteq \operatorname{ker}(g)$. This contradicts that the given sequence is exact.

As none of the $X_{i}$ satisfy $i$ ), we can separate these modules into those supported on $M(u)$ and those supported on $M(v)$. We denote the former modules by $\left\{M\left(u^{(j)}\right)\right\}_{j=1}^{s}$ and the latter by $\left\{M\left(v^{(\ell)}\right)\right\}_{\ell=1}^{t}$. 
Suppose $M\left(u^{(j)}\right)$ satisfies $\left.i i\right)$. Then there exist $M\left(u^{\left(j^{\prime}\right)}\right)$ for some $j^{\prime} \neq j$ such that $u^{(j)} \stackrel{\beta}{\longleftrightarrow} u^{\left(j^{\prime}\right)}$ is a string in $\Lambda_{T}$ supported on $u$. Thus, if $u^{(j)} \stackrel{\beta}{\longleftarrow} u^{\left(j^{\prime}\right)}\left(\operatorname{resp} ., u^{(j)} \stackrel{\beta}{\longrightarrow} u^{\left(j^{\prime}\right)}\right)$ is a string in $\Lambda_{T}$, we have that $\operatorname{Hom}_{\Lambda_{T}}\left(M(u), M\left(u^{(j)}\right)\right)=0\left(\right.$ resp., $\left.\operatorname{Hom}_{\Lambda_{T}}\left(M(u), M\left(u^{\left(j^{\prime}\right)}\right)\right)=0\right)$. This implies that there exists a summand $M\left(u^{\left(j^{\prime \prime}\right)}\right)$ of $X$ such that $M\left(u^{\left(j^{\prime \prime}\right)}\right) \cap \operatorname{im}(f)=0$. However, $M\left(u^{\left(j^{\prime \prime}\right)}\right) \subseteq \operatorname{ker}(g)$ since $\operatorname{supp}\left(M\left(u^{\left(j^{\prime \prime}\right)}\right)\right) \cap \operatorname{supp}(M(v))=\emptyset$. This contradicts that the given sequence is exact. The proof that there are no summands $M\left(v^{(\ell)}\right)$ of $X$ that satisfy iii) is similar so we omit it.

Lemma 5.7. Let $M(u), M(v) \in \operatorname{ind}\left(\Lambda_{T}\right.$-mod) where $s_{u}$ and $s_{v}$ have no common end points. Let $0 \rightarrow M(u) \stackrel{f}{\rightarrow} X \stackrel{g}{\rightarrow} M(v) \rightarrow 0$ be a nonsplit extension where $\operatorname{supp}(M(u)) \cap$ $\operatorname{supp}(M(v)) \neq \emptyset$, and let $w$ denote the unique maximal string supported on $\operatorname{supp}(M(u)) \cap$ $\operatorname{supp}(M(v))$. Let $X=\oplus_{i=1}^{k} X_{i}$ be a direct sum decomposition of $X$ into indecomposables and write $u=u^{(1)} \leftrightarrow w \leftrightarrow u^{(2)}$ and $v=v^{(1)} \leftrightarrow w \leftrightarrow v^{(2)}$ for some strings $u^{(1)}, u^{(2)}, v^{(1)}$, and $v^{(2)}$ in $\Lambda_{T}$, some of which may be empty. Then the following hold:

(i) $X$ is not indecomposable.

(ii) There is no $X_{i}$ such that $\operatorname{supp}\left(X_{i}\right) \cap \operatorname{supp}(M(x)) \neq \emptyset$ for any $x \in\left\{w, u^{(1)}, u^{(2)}\right\}$, assuming that both $u^{(1)}$ and $u^{(2)}$ are nonempty strings.

(iii) There is no $X_{i}$ such that $\operatorname{supp}\left(X_{i}\right) \cap \operatorname{supp}(M(x)) \neq \emptyset$ for any $x \in\left\{w, v^{(1)}, v^{(2)}\right\}$, assuming that both $v^{(1)}$ and $v^{(2)}$ are nonempty strings.

(iv) There is no $X_{i}$ such that $\operatorname{supp}\left(X_{i}\right) \subsetneq \operatorname{supp}(M(x))$ where $x \in\left\{u^{(1)}, u^{(2)}, v^{(1)}, v^{(2)}\right\}$. Thus, each $X_{i}$ satisfies $\operatorname{supp}\left(X_{i}\right) \cap \operatorname{supp}(M(w)) \neq \emptyset$.

(v) If $X_{i}$ and $x \in\left\{w, u^{(1)}, u^{(2)}, v^{(1)}, v^{(2)}\right\}$ satisfy $\operatorname{supp}\left(X_{i}\right) \cap \operatorname{supp}(M(x)) \neq \emptyset$, then $\operatorname{supp}(M(x)) \subseteq \operatorname{supp}\left(X_{i}\right)$.

Proof. We first show that each $X_{i}$ satisfies $X_{i} ¥ M(u)$ and $X_{i} ¥ M(v)$. Without loss of generality, suppose a summand $X_{i}$ of $X$ satisfies $X_{i} \cong M(u)$. Since $s_{u}$ and $s_{v}$ have no common end points, $\operatorname{im}(f)=X_{i}$. By dimension considerations and the fact that $g$ is surjective, $M(v)$ is also a summand of $X$. Thus, the given sequence is split, a contradiction.

(i) We observe that by exactness, $\underline{\operatorname{dim}}(X)=\underline{\operatorname{dim}}(M(u))+\underline{\operatorname{dim}}(M(v))$. Since $\operatorname{supp}(M(u)) \cap \operatorname{supp}(M(v)) \neq \emptyset$, Corollary $4.3 \overline{(1)}$ implies that $X$ is not a string module and therefore not indecomposable.

(ii) Suppose that such an $X_{i}$ exists. Then $\operatorname{supp}(M(w)) \subseteq \operatorname{supp}\left(X_{i}\right)$. Now note that since $X_{i} \nsucceq M(u)$ and $X_{i} \not M(v)$ we can assume without loss of generality, that $\operatorname{supp}\left(X_{i}\right) \cap$ $\operatorname{supp}\left(M\left(u^{(1)}\right)\right) \subsetneq \operatorname{supp}\left(M\left(u^{(1)}\right)\right)$ and $\operatorname{supp}\left(X_{i}\right) \cap \operatorname{supp}\left(M\left(u^{(1)}\right)\right) \neq \emptyset$. This implies that we can write $u^{(1)}=x^{(1)} \leftrightarrow x^{(2)}$ for some nonempty strings $x^{(1)}$ and $x^{(2)}$ in $\Lambda_{T}$ where $\operatorname{supp}\left(M\left(x^{(2)}\right)\right)=\operatorname{supp}\left(X_{i}\right) \cap \operatorname{supp}\left(M\left(u^{(1)}\right)\right)$ and $u=x^{(1)} \leftrightarrow x^{(2)} \leftrightarrow w \leftrightarrow u^{(2)}$.

Suppose $u^{(1)}=x^{(1)} \leftarrow x^{(2)}$. Now write $x^{(1)}=x_{1}^{(1)} \leftrightarrow x_{2}^{(1)} \leftrightarrow \cdots \leftrightarrow x_{\ell}^{(1)}$ so that

$$
u^{(1)}=x^{(1)} \leftarrow x^{(2)}=\left(x_{1}^{(1)} \leftrightarrow x_{2}^{(1)} \leftrightarrow \cdots \leftrightarrow x_{\ell}^{(1)}\right) \leftarrow x^{(2)} .
$$

In this case, $\operatorname{Hom}_{\Lambda_{T}}\left(M(u), X_{j}\right)=0$ if $X_{j}$ is any summand of $X$ where $\operatorname{supp}\left(X_{j}\right) \subseteq$ $\operatorname{supp}\left(M\left(x^{(1)}\right)\right)$ and $x_{\ell}^{(1)} \in \operatorname{supp}\left(X_{j}\right)$. Thus, any such $X_{j}$ satisfies $X_{j} \cap \operatorname{im}(f)=0$. One also observes that $\operatorname{supp}\left(M\left(x^{(1)}\right)\right) \cap \operatorname{supp}(M(v))=\emptyset$ so $\operatorname{Hom}_{\Lambda_{T}}\left(X_{j}, M(v)\right)=0$. Therefore, any such $X_{j} \subseteq \operatorname{ker}(g)$. This means that if such a summand $X_{j}$ exists, then the given sequence is not exact.

We show that there must be a summand $X_{j}$ of $X$ satisfying $\operatorname{supp}\left(X_{j}\right) \subseteq \operatorname{supp}\left(M\left(x^{(1)}\right)\right)$ and whose string contains $x_{\ell}^{(1)}$. First note that by the exactness of the given sequence, there must exist a summand $X_{j}$ of $X$ whose support contains $x_{\ell}^{(1)}$ and thus intersects $\operatorname{supp}\left(M\left(x^{(1)}\right)\right)$. It is enough to show that, without loss of generality, there is no string $y$ 
in $\Lambda_{T}$ such that $\operatorname{supp}(M(y)) \cap \operatorname{supp}\left(M\left(x^{(1)}\right)\right) \neq \emptyset$ and $\operatorname{supp}(M(y)) \cap \operatorname{supp}\left(M\left(v^{(1)}\right)\right) \neq \emptyset$. To show this, it is enough to observe that the segments $s_{x^{(1)}}$ and $s_{v^{(1)}}$ have no common vertices. The latter follows from the fact that $x^{(2)}$ is a nonempty string. We obtain a contradiction.

We now have that $u^{(1)}=x^{(1)} \rightarrow x^{(2)}$. This implies that $\operatorname{Hom}_{\Lambda_{T}}\left(M(u), X_{i}\right)=0$. Let us express $X_{i}$ as $X_{i}=\left(\left(V_{k}\right)_{k \in Q_{0}},\left(\varphi_{\alpha}\right)_{\alpha \in Q_{1}}\right)$. By exactness and dimension considerations, the module $X_{i}$ is the only summand of $X$ satisfying $\operatorname{supp}\left(X_{i}\right) \cap \operatorname{supp}\left(M\left(x^{(2)}\right)\right) \neq \emptyset$. Thus, there exists $\lambda \in V_{k}$ that is nonzero with $k \in \operatorname{supp}\left(X_{i}\right) \cap \operatorname{supp}\left(M\left(x^{(2)}\right)\right)$ and $\lambda \notin \operatorname{im}(f)$. However, $\lambda$ is also a nonzero element of $M(u)$, and this contradicts that $f$ is injective.

(iii) The proof of this assertion is similar to the proof of assertion (ii) so we omit it.

(iv) It suffices to show that there does not exist a summand $X_{i}$ of $X$ such that $\operatorname{supp}\left(X_{i}\right) \subsetneq$ $\operatorname{supp}\left(M\left(v^{(1)}\right)\right)$. Suppose there exists such a summand $X_{i}$. Then there exist summands $M(x)$ and $M(y)$ of $X$ such that $x \leftrightarrow y$ is a string in $\Lambda_{T}$ where $\operatorname{supp}(M(x)) \subsetneq \operatorname{supp}\left(M\left(v^{(1)}\right)\right)$ and $\operatorname{supp}(M(y)) \cap \operatorname{supp}\left(M\left(v^{(1)}\right)\right) \neq \emptyset$. If $(x \leftrightarrow y)=(x \leftarrow y)$, then $\operatorname{Hom}_{\Lambda_{T}}(M(y), M(v))=0$. Let us express $M(y)$ as $M(y)=\left(\left(V_{k}\right)_{k \in Q_{0}},\left(\varphi_{\alpha}\right)_{\alpha \in Q_{1}}\right)$. Then any nonzero $\lambda \in V_{k}$ where $k \in \operatorname{supp}(M(y)) \cap \operatorname{supp}\left(M\left(v^{(1)}\right)\right)$ satisfies $\lambda \in \operatorname{ker}(g)$. Since $\lambda$ does not belong to any summand besides $M(y)$, we have that $g$ is not surjective, a contradiction. If instead $(x \leftrightarrow y)=$ $(x \rightarrow y)$, then $\operatorname{Hom}_{\Lambda_{T}}(M(x), M(v))=0$. Similarly, this implies that $M(x) \subseteq \operatorname{ker}(g)$, which contradicts that $g$ is surjective.

$v$ ) We first prove the assertion for any $x \in\left\{u^{(1)}, u^{(2)}, v^{(1)}, v^{(2)}\right\}$. It suffices to prove this for $x=v^{(1)}$. Suppose that there exists $X_{i}$ such that $\operatorname{supp}\left(X_{i}\right) \cap \operatorname{supp}\left(M\left(v^{(1)}\right)\right) \neq \varnothing$ and $\operatorname{supp}\left(M\left(v^{(1)}\right)\right) \nsubseteq \subseteq \operatorname{supp}\left(X_{i}\right)$. By $\left.i v\right)$, we have that $\operatorname{supp}\left(X_{i}\right) \cap \operatorname{supp}(M(w)) \neq \emptyset$. Now by exactness of the given sequence, there exists another summand $X_{j}$ of $X$ such that $\operatorname{supp}\left(X_{j}\right) \subseteq \operatorname{supp}\left(M\left(v^{(1)}\right)\right) \backslash \operatorname{supp}\left(X_{i}\right) \subseteq \operatorname{supp}\left(M\left(v^{(1)}\right)\right)$. This contradicts $\left.i v\right)$.

Next, we suppose $x=w$. By assertion $i v)$, each summand $X_{i}$ satisfies $\operatorname{supp}\left(X_{i}\right) \cap$ $\operatorname{supp}(M(w)) \neq \emptyset$. Thus, it is enough to show that there are no summands $X_{i}$ such that $\operatorname{supp}\left(X_{i}\right) \subsetneq \operatorname{supp}(M(w))$. Suppose there exists such a summand $X_{i}=M\left(y^{(2)}\right)$. We can assume, without loss of generality, that there is another summand $X_{j}=M\left(y^{(1)}\right)$ of $X$ such that

- $y^{(1)} \leftrightarrow y^{(2)}$ is a string in $\Lambda_{T}$,

- $\operatorname{supp}\left(M\left(y^{(1)}\right)\right) \cap \operatorname{supp}\left(M\left(v^{(1)}\right)\right) \neq \emptyset$,

- $\operatorname{supp}\left(M\left(y^{(1)}\right)\right) \cap \operatorname{supp}(M(w)) \neq \emptyset$.

Suppose that $\left(y^{(1)} \leftrightarrow y^{(2)}\right)=\left(y^{(1)} \rightarrow y^{(2)}\right)$. Then $\operatorname{Hom}_{\Lambda_{T}}\left(M\left(y^{(1)}\right), M(v)\right)=0$. Let us express $M\left(y^{(1)}\right)$ as $M\left(y^{(1)}\right)=\left(\left(V_{k}\right)_{k \in Q_{0}},\left(\varphi_{\alpha}\right)_{\alpha \in Q_{1}}\right)$. Then any nonzero $\lambda \in V_{k}$ where $k \in$ $\operatorname{supp}\left(M\left(y^{(1)}\right)\right) \cap \operatorname{supp}\left(M\left(v^{(1)}\right)\right)$ satisfies $\lambda \in \operatorname{ker}(g)$. Since $M\left(y^{(1)}\right)$ is the only summand containing $\lambda$, this contradicts that $g$ is surjective.

Now suppose $\left(y^{(1)} \leftrightarrow y^{(2)}\right)=\left(y^{(1)} \leftarrow y^{(2)}\right)$ and write $y^{(2)}=y_{1}^{(2)} \leftrightarrow \cdots \leftrightarrow y_{\ell}^{(2)}$. Then $\operatorname{Hom}_{\Lambda_{T}}\left(M\left(y^{(2)}\right), M(v)\right)=0$. This means that any other summand $M\left(y^{(3)}\right)$ of $X$ where $\left(y^{(1)} \leftarrow y^{(3)}\right)$ is a string in $\Lambda_{T}$ and $y_{1}^{(2)} \in \operatorname{supp}\left(M\left(y^{(3)}\right)\right)$ has the property that $\operatorname{Hom}_{\Lambda_{T}}\left(M\left(y^{(3)}\right), M(v)\right)=0$. Since $M\left(y^{(1)}\right)$ is the only summand of $X$ whose support inter$\operatorname{sects} \operatorname{supp}\left(M\left(y^{(1)}\right)\right) \cap \operatorname{supp}\left(M\left(v^{(1)}\right)\right)$ and $\operatorname{since} \operatorname{supp}\left(M\left(y^{(1)}\right)\right) \subseteq \operatorname{supp}(M(v))$, we have that there is an inclusion $M\left(y^{(1)}\right) \hookrightarrow M(v)$. Since the given sequence is exact, there must exist a summand $M(z)=\left(\left(V_{k}\right)_{k \in Q_{0}},\left(\varphi_{\alpha}\right)_{\alpha \in Q_{1}}\right)$ of $X$ where $z$ satisfies

- $\operatorname{supp}(M(z)) \cap \operatorname{supp}\left(M\left(y^{(1)}\right)\right) \neq \emptyset$ where any nonzero $\lambda \in V_{k}$ with $k \in \operatorname{supp}(M(z)) \cap$ $\operatorname{supp}\left(M\left(y^{(1)}\right)\right)$ satisfies $\lambda \notin \operatorname{ker}(g)=\operatorname{im}(f)$, and

- $\operatorname{supp}(M(z)) \cap \operatorname{supp}\left(M\left(y^{(2)}\right)\right) \neq \varnothing$ where any nonzero $\lambda \in V_{k}$ with $k \in \operatorname{supp}(M(z)) \cap$ $\operatorname{supp}\left(M\left(y^{(2)}\right)\right)$ satisfies $\lambda \in \operatorname{im}(f)$. 
However, since $\left(y^{(1)} \leftrightarrow y^{(2)}\right)=\left(y^{(1)} \leftarrow y^{(2)}\right)$ there are no homomorphisms from $M(u)$ to $M(z)$ satisfying these properties. Thus, there are no summands $X_{i}$ of $X$ such that $\operatorname{supp}\left(X_{i}\right) \subsetneq$ $\operatorname{supp}(M(w))$.

Corollary 5.8. Let $M(u), M(v) \in \operatorname{ind}\left(\Lambda_{T}\right.$-mod $)$ where $s_{u}$ and $s_{v}$ have no common end points. Let $0 \rightarrow M(u) \stackrel{f}{\rightarrow} X \stackrel{g}{\rightarrow} M(v) \rightarrow 0$ be a nonsplit extension where $\operatorname{supp}(M(u)) \cap$ $\operatorname{supp}(M(v)) \neq \emptyset$, and let $w$ denote the unique maximal string supported on $\operatorname{supp}(M(u)) \cap$ $\operatorname{supp}(M(v))$. Let $X=\oplus_{i=1}^{k} X_{i}$ be a direct sum decomposition of $X$ into indecomposables and write $u=u^{(1)} \leftrightarrow w \leftrightarrow u^{(2)}$ and $v=v^{(1)} \leftrightarrow w \leftrightarrow v^{(2)}$ for some strings $u^{(1)}, u^{(2)}, v^{(1)}$, and $v^{(2)}$ in $\Lambda_{T}$ some of which may be empty. Then $X=M\left(u^{(1)} \leftrightarrow w \leftrightarrow v^{(2)}\right) \oplus M\left(v^{(1)} \leftrightarrow w \leftrightarrow u^{(2)}\right)$.

Proof. By Lemma 5.7 (i), $X$ has at least two indecomposable summands. By Lemma $5.7 i v$ ) and v), $X$ has exactly two summands, $M(y)$ and $M(z)$, where $\operatorname{supp}(M(w)) \subseteq$ $\operatorname{supp}(M(y))$ and $\operatorname{supp}(M(w)) \subseteq \operatorname{supp}(M(z))$. By exactness of the given sequence and by Lemma $5.7 v$ ), for any $x \in\left\{u^{(1)}, u^{(2)}, v^{(1)}, v^{(2)}\right\}$ we have that $\operatorname{supp}(M(x))$ is contained in $\operatorname{supp}(M(y))$ or $\operatorname{supp}(M(z))$. By combining Lemma $5.7 i i)$ and iii), we have that $M(y)=$ $M\left(u^{(1)} \leftrightarrow w \leftrightarrow v^{(2)}\right)$ and $M(z)=M\left(v^{(1)} \leftrightarrow w \leftrightarrow u^{(2)}\right)$.

LEMMA 5.9. Let $M(u), M(v) \in$ ind $\left(\Lambda_{T}\right.$-mod $)$ where $s_{u}$ and $s_{v}$ either share an end point and agree along a segment or they have a common vertex that is an end point of at most one of $s_{u}$ and $s_{v}$. If $0 \rightarrow M(u) \stackrel{f}{\rightarrow} X \stackrel{g}{\rightarrow} M(v) \rightarrow 0$ is an extension and $X=\bigoplus_{i=1}^{k} X_{i}$ is a direct sum decomposition into indecomposables, then the following hold:

(i) $X$ is not indecomposable.

(ii) There is no $X_{i}$ such that $\operatorname{supp}\left(X_{i}\right) \subsetneq \operatorname{supp}(M(x))$ where $x \in\{u, v\}$.

Proof. Only Lemma 5.7 (ii) and (iii) relied on the assumption that the given extension was nonsplit. Thus, one proves these assertions by adapting the proofs of Lemmas $5.7(i)$, (iv), and (v), since these did not depend on Lemma 5.7 (ii) and (iii).

6. Oriented flip graphs and torsion-free classes. In this section, we recall the definition of torsion-free classes and their lattice structure. After that, we show that the oriented flip graph of $T$ is isomorphic as a poset to the lattice of torsion-free classes of $\Lambda_{T}$ ordered by inclusion and torsion classes of $\Lambda_{T}$ ordered by reverse inclusion.

Let $\Lambda$ be a finite-dimensional $\mathbb{k}$-algebra. A full, additive subcategory $\mathcal{C} \subseteq \Lambda$-mod is extension closed if for any objects $X, Y \in \mathcal{C}$ satisfying $0 \rightarrow X \rightarrow Z \rightarrow Y \rightarrow 0$ one has $Z \in \mathcal{C}$. We say $\mathcal{C}$ is quotient closed (resp., submodule closed) if for any $X \in \mathcal{C}$ satisfying $X \stackrel{g}{\longrightarrow} Z$ where $g$ is a surjection (resp., $Z \stackrel{f}{\longrightarrow} X$ where $f$ is an injection), then $Z \in \mathcal{C}$. A full, additive subcategory $\mathcal{T} \subseteq \Lambda$-mod is called a torsion class if $\mathcal{T}$ is quotient closed and extension closed. Dually, a full, additive subcategory $\mathcal{F} \subseteq \Lambda$-mod is called a torsion-free class if $\mathcal{F}$ is extension closed and submodule closed.

Let tors $(\Lambda)$ (resp., torsf $(\Lambda))$ denote the lattice of torsion classes (resp., of torsion-free classes) of $\Lambda$, ordered by inclusion. We have the following proposition, which shows that a torsion class of $\Lambda$ uniquely determines a torsion-free class of $\Lambda$ and vice versa. This statement is a classical result in tilting theory; see, e.g., [25, Prop. 1.1 a)].

PROPOSITION 6.1. The maps

$$
\begin{aligned}
\operatorname{tors}(\Lambda) & \stackrel{(-)^{\perp}}{\longrightarrow} \operatorname{torsf}(\Lambda) \\
\mathcal{T} & \longmapsto \mathcal{T}^{\perp}:=\left\{X \in \Lambda-\bmod : \operatorname{Hom}_{\Lambda}(\mathcal{T}, X)=0\right\}
\end{aligned}
$$


and

$$
\begin{aligned}
\operatorname{tors} f(\Lambda) & \stackrel{\perp_{(-)}}{\longrightarrow} \operatorname{tors}(\Lambda) \\
\mathcal{F} & \longmapsto \\
& { }^{\perp} \mathcal{F}:=\left\{X \in \Lambda \text {-mod: } \operatorname{Hom}_{\Lambda}(X, \mathcal{F})=0\right\}
\end{aligned}
$$

are inverse bijections.

Given $\mathcal{T}$ a torsion class and $\mathcal{F}$ its corresponding torsion-free class, we say that the data $(\mathcal{T}, \mathcal{F})$ is a torsion pair.

Proposition 6.2 ([25, Prop. 1.3]). Let $\Lambda$ be a finite-dimensional algebra. Then tors $(\Lambda)$ and tors $f(\Lambda)$ are complete lattices. The join and meet operations are described as follows.

(1) Let $\left\{\mathcal{T}_{i}\right\}_{i \in I} \subseteq$ tors $(\Lambda)$ be a collection of torsion classes. Then we have $\bigwedge_{i \in I} \mathcal{T}_{i}=$ $\bigcap_{i \in I} \mathcal{T}_{i}$ and $\bigvee_{i \in I} \mathcal{T}_{i}={ }^{\perp}\left(\bigcap_{i \in I} \mathcal{T}_{i}^{\perp}\right)$.

(2) Let $\left\{\mathcal{F}_{i}\right\}_{i \in I} \subseteq \operatorname{tors} f(\Lambda)$ be a collection of torsion-free classes. Then we have $\bigwedge_{i \in I}$ $\mathcal{F}_{i}=\bigcap_{i \in I} \mathcal{F}_{i}$ and $\bigvee_{i \in I} \mathcal{F}_{i}=\left(\bigcap_{i \in I}^{\perp} \mathcal{F}_{i}\right)^{\perp}$.

LEMMA 6.3 ([25, Prop. 1.4 a), c)]). The maps

$$
\begin{aligned}
\operatorname{tors}(\Lambda) & \stackrel{D(-)}{\longrightarrow} \operatorname{tors} f\left(\Lambda^{o p}\right) \cong \operatorname{tors} f(\Lambda)^{o p} \\
\mathcal{T} & \longmapsto D \mathcal{T}
\end{aligned}
$$

and

$$
\begin{aligned}
\operatorname{torsf}(\Lambda) & \stackrel{D(-)}{\longrightarrow} \operatorname{tors}\left(\Lambda^{o p}\right) \cong \operatorname{tors}(\Lambda)^{o p} \\
\mathcal{F} & \longmapsto D \mathcal{F}
\end{aligned}
$$

are isomorphisms of lattices where $D(-):=\operatorname{Hom}_{\mathbb{k}}(-, \mathbb{k})$ is the standard duality. Furthermore, the functor $D\left((-)^{\perp}\right): \operatorname{tors}(\Lambda) \rightarrow \operatorname{tors}\left(\Lambda^{o p}\right)$ is an anti-isomorphism of posets.

We now return to the case where $\Lambda=\Lambda_{T}$ is a tiling algebra for a tree $T$ embedded in a disk. For a string $w=x_{1} \stackrel{\alpha_{1}}{\leftrightarrow} \cdots \stackrel{\alpha_{\ell-1}}{\leftrightarrow} x_{\ell}$, the indecomposable submodules of $M(w)$ are those $M(u)$ for which $u=x_{i} \leftrightarrow \cdots \leftrightarrow x_{j}$ is a substring of $w$ such that

- if $i>1$ then $x_{i-1} \stackrel{\alpha_{i-1}}{\leftarrow} x_{i}$, and

- if $j<\ell$ then $x_{j} \stackrel{\alpha_{j}}{\rightarrow} x_{j+1}$.

Translating to conditions on the segments $s_{w}$ and $s_{u}$ and recalling the definition of $C_{s}$ in Section 3.1, we deduce that

$$
C_{s}=\left\{s^{\prime}: M\left(s^{\prime}\right) \text { is a submodule of } M(s)\right\} .
$$

THEOREM 6.4. For any tree $T$, we have that $\overrightarrow{F G}(T) \cong \operatorname{tors} f\left(\Lambda_{T}\right)$ and $\overrightarrow{F G}(T) \cong$ tors $\left(\Lambda_{T}\right)^{\text {op }}$ where tors $\left(\Lambda_{T}\right)^{\text {op }}$ denotes the lattice of torsion classes ordered by reverse inclusion.

Proof. We claim that the map

$$
\begin{aligned}
\overrightarrow{F G}(T)=\pi_{\downarrow}(\operatorname{Bic}(T)) & \stackrel{\zeta}{\longrightarrow} \operatorname{torsf}\left(\Lambda_{T}\right) \\
X & \longmapsto \mathcal{F}:=\operatorname{add}\left(\oplus_{s_{u}} M(u): s_{u} \in X\right)
\end{aligned}
$$


is an isomorphism of posets where $\operatorname{add}\left(\oplus_{i=1}^{k} X_{i}\right)$ denotes the smallest full, additive subcategory of $\Lambda_{T}$-mod closed under taking summands of $\oplus_{i=1}^{k} X_{i}$. Furthermore, we claim that the inverse of this map is given by

$$
\begin{aligned}
& \operatorname{torsf}\left(\Lambda_{T}\right) \stackrel{\delta}{\longrightarrow} \pi_{\downarrow}(\operatorname{Bic}(T)) \\
& \mathcal{F}=\operatorname{add}\left(\oplus_{i \in[k]} M\left(w^{(i)}\right)\right) \longmapsto\left\{s_{w^{(1)}}, \ldots, s_{w^{(k)}}\right\} .
\end{aligned}
$$

It is clear that these maps are order-preserving inverses of each other. So, it is enough to show that $\zeta(X)$ is a torsion-free class and that $\delta(\mathcal{F}) \in \pi_{\downarrow}(\operatorname{Bic}(T))$.

To show that $\delta(\mathcal{F}) \in \pi_{\downarrow}(\operatorname{Bic}(T))$ where $\mathcal{F}=\operatorname{add}\left(\oplus_{i \in[k]} M\left(w^{(i)}\right)\right)$, we first show that $\left\{s_{w^{(1)}}, \ldots, s_{w^{(k)}}\right\}$ is biclosed. Let $s_{u}, s_{v} \in\left\{s_{w^{(1)}}, \ldots, s_{w^{(k)}}\right\}$ and assume $s_{u} \circ s_{v} \in \operatorname{Seg}(T)$. Then, up to reversing the roles of $u$ and $v, u \leftarrow v$ is a string in $\Lambda_{T}$ so there is an extension $0 \rightarrow M(u) \rightarrow M(u \leftarrow v) \rightarrow M(v) \rightarrow 0$. Since $\mathcal{F}$ is extension closed, $M(u \leftarrow v) \in \mathcal{F}$ so $s_{u} \circ s_{v}=s_{(u \leftarrow v)} \in\left\{s_{w^{(1)}}, \ldots, s_{w^{(k)}}\right\}$. Thus, $\left\{s_{w^{(1)}}, \ldots, s_{w^{(k)}}\right\}$ is closed. Since $\mathcal{F}$ is submodule closed, there are no extensions of the form $0 \rightarrow M(u) \rightarrow M(u \leftarrow v) \rightarrow M(v) \rightarrow 0$ where $s_{u}, s_{v} \notin\left\{s_{w^{(1)}}, \ldots, s_{w^{(k)}}\right\}$, but $s_{(u \leftarrow v)} \in\left\{s_{w^{(1)}}, \ldots, s_{w^{(k)}}\right\}$. Thus, $\left\{s_{w^{(1)}}, \ldots, s_{w^{(k)}}\right\}$ is co-closed.

We now show that $\left\{s_{w^{(1)}}, \ldots, s_{w^{(k)}}\right\}=\pi_{\downarrow}\left(\left\{s_{w^{(1)}}, \ldots, s_{w^{(k)}}\right\}\right)$. We only need to show that the former is a subset of the latter. Given any $s_{w^{(i)}} \in\left\{s_{W^{(1)}}, \ldots, s_{w^{(k)}}\right\}$, we know that every submodule of $M\left(w^{(i)}\right)$ belongs to $\mathcal{F}$ since $\mathcal{F}$ is a torsion-free class. Thus, $C_{s_{w^{(i)}}} \subseteq\left\{s_{w^{(1)}}, \ldots, s_{w^{(k)}}\right\}$ so $s_{w^{(i)}} \in \pi_{\downarrow}\left(\left\{s_{w^{(1)}}, \ldots, s_{w^{(k)}}\right\}\right)$. It follows that $\delta(\mathcal{F}) \in \pi_{\downarrow}(\operatorname{Bic}(T))$.

Next, we show that $\mathcal{F}:=\operatorname{add}\left(\oplus_{s_{u}} M(u): s_{u} \in X\right)$ is a torsion-free class given $X \in$ $\pi_{\downarrow}(\operatorname{Bic}(T))$. We begin by showing that it is submodule closed. Assume that there is an inclusion $M(v) \hookrightarrow M(u)$ where $M(u) \in \mathcal{F}$. This implies that $C_{S_{u}} \subseteq X$, since $s_{u} \in X$ and $X \in \pi_{\downarrow}(\operatorname{Bic}(T))$.

Write $s_{u}=\left(x_{0}, \ldots, x_{\ell}\right)$ and orient this segment from $x_{0}$ to $x_{\ell}$. Let $s_{v}=\left(x_{i}, \ldots, x_{j}\right)$ where we can assume that $0<i$ and $j<\ell$. The inclusion $M(v) \hookrightarrow M(u)$ implies that $u=u^{(1)} \rightarrow v \leftarrow u^{(2)}$ for some nonempty strings $u^{(1)}$ and $u^{(2)}$ in $\Lambda_{T}$. Now we have that $s_{v}$ turns right (resp., left) at $x_{i}$ (resp., at $x_{j}$ ). Thus, $s_{v} \in C_{s_{u}} \subseteq X$. We obtain that $M(v) \in \mathcal{F}$.

Now suppose $f: M(v) \hookrightarrow N=\oplus_{i \in[k]} M\left(w^{(i)}\right)^{a_{i}}$ for some $a_{i} \geq 0$ and $M(v)$ does not include into any summand of $N$. Furthermore, suppose any indecomposable $M(u)$ with $\operatorname{dim}_{\mathbb{k}}(M(u))<\operatorname{dim}_{\mathbb{k}}(M(v))$ that includes into an object of $\mathcal{F}$ belongs to $\mathcal{F}$. Let $M\left(w^{(i)}\right)$ be a summand of $N$ where the component map $g: M(v) \rightarrow M\left(w^{(i)}\right)$ of $f$ is nonzero. By Lemma 5.3, we can assume that there exists a nonempty string $w$ in $\Lambda_{T}$ not equal to $u$ or $w^{(i)}$ such that $M(v) \rightarrow M(w) \hookrightarrow M\left(w^{(i)}\right)$. By the previous two paragraphs, $M(w) \in \mathcal{F}$.

Express $v$ as $v=v^{(1)} \leftarrow w \rightarrow v^{(2)}$ where, without loss of generality, both $v^{(1)}$ and $v^{(2)}$ are nonempty. This implies that $M\left(v^{(i)}\right) \hookrightarrow N$ so $M\left(v^{(i)}\right) \in \mathcal{F}$ for $i=1,2$ since $\operatorname{dim}_{\mathbb{k}}\left(M\left(v^{(i)}\right)\right)<\operatorname{dim}_{\mathbb{k}}(M(v))$. Observe that we have an extension $0 \rightarrow M\left(v^{(2)}\right) \rightarrow M(w \rightarrow$ $\left.v^{(2)}\right) \rightarrow M(w) \rightarrow 0$, which shows that $M\left(w \rightarrow v^{(2)}\right) \in \mathcal{F}$ since $s_{\left(w \rightarrow v^{(2)}\right)}=s_{w} \circ s_{v^{(2)}} \in X$. This implies that we have an extension $0 \rightarrow M\left(v^{(1)}\right) \rightarrow M(v) \rightarrow M\left(w \rightarrow v^{(2)}\right) \rightarrow 0$, which shows that $M(v) \in \mathcal{F}$ since $s_{v}=s_{v^{(1)}} \circ s_{w} \circ s_{v^{(2)}} \in X$. We conclude that $\mathcal{F}$ is submodule closed.

Next, consider the subcategory filt $(\mathcal{F}) \subseteq \Lambda_{T}$-mod consisting of objects $M \in \Lambda_{T}$-mod with a filtration $0=M_{0} \subseteq M_{1} \subseteq \cdots \subseteq M_{k}=M$ where for each $i \in\{1, \ldots, k\}$ there exists $M\left(u^{i}\right) \in\left\{M(u): s_{u} \in X\right\}$ such that $M_{i} / M_{i-1} \hookrightarrow M\left(u^{i}\right)$. Clearly, $\mathcal{F} \subseteq$ filt $(\mathcal{F})$. Additionally, it is known that $f i l t(\mathcal{F})$ is a torsion-free class.

To complete the proof, we show that $\operatorname{filt}(\mathcal{F}) \subseteq \mathcal{F}$. We show that any indecomposable object of filt $(\mathcal{F})$ belongs to $\mathcal{F}$. Let $M(w) \in \operatorname{ind}($ filt $(\mathcal{F}))$ and let $0=M_{0} \subseteq M_{1} \subseteq \cdots \subseteq$ $M_{k}=M(w)$ be a filtration witnessing that $M(w) \in f i l t(\mathcal{F})$. We show by induction that each 
$M_{i} \in \mathcal{F}$. For the base case, observe that $M_{1} \in \mathcal{F}$ because $\mathcal{F}$ is submodule closed. So, we assume that $M_{i} \in \mathcal{F}$ and prove that $M_{i+1} \in \mathcal{F}$.

Since $\mathcal{F}$ is submodule closed, $M_{i+1} / M_{i} \in \mathcal{F}$. Now, write $M_{i}=\oplus_{r=1}^{\ell} M\left(u^{(r)}\right)$ and $M_{i+1} / M_{i}=\oplus_{t=1}^{m} M\left(v^{(t)}\right)$ where $M\left(u^{(r)}\right), M\left(v^{(t)}\right) \in\left\{M(u): s_{u} \in \pi_{\downarrow}(X)\right\}$ for all $r \in$ $\{1, \ldots, \ell\}$ and all $t \in\{1, \ldots, m\}$. Moreover, by Corollary 4.3(1) and since $M_{i}$ is a submodule of $M(w), \operatorname{supp}\left(M\left(u^{(r)}\right)\right) \cap \operatorname{supp}\left(M\left(u^{\left(r^{\prime}\right)}\right)\right)=\emptyset$ for any distinct $r, r^{\prime} \in\{1, \ldots, \ell\}$. Similarly, $\operatorname{supp}\left(M\left(v^{(t)}\right)\right) \cap \operatorname{supp}\left(M\left(v^{\left(t^{\prime}\right)}\right)\right)=\emptyset$ for any distinct $t, t^{\prime} \in\{1, \ldots, m\}$. Since $0 \rightarrow \oplus_{r=1}^{\ell} M\left(u^{(r)}\right) \rightarrow M_{i+1} \rightarrow \oplus_{t=1}^{m} M\left(v^{(t)}\right) \rightarrow 0$ is an extension and $M_{i+1}$ is a submodule of $M(w)$, we see that $M_{i+1}$ is a direct sum of submodules $M\left(w^{\prime}\right) \subseteq M(w)$ with pairwise disjoint supports such that each segment $s_{w^{\prime}}$ is the composition of segments in $\left\{s_{u^{(r)}}, s_{v^{(t)}}\right\}_{1 \leq r \leq \ell, 1 \leq t \leq m}$. Since $X$ is closed, each summand of $M_{i+1}$ belongs to $\mathcal{F}$. We obtain that $M_{i+1} \in \mathcal{F}$. Since $\mathcal{F}=$ filt $(\mathcal{F})$, we know that $\mathcal{F} \in \operatorname{torsf}\left(\Lambda_{T}\right)$.

7. Noncrossing tree partitions and wide subcategories. In this section, we show that noncrossing tree partitions of a tree $T$ provide a combinatorial model for the wide subcategories of $\Lambda_{T}$-mod.

If $\Lambda$ is a finite-dimensional $\mathbb{k}$-algebra, we say that a full, additive subcategory $\mathcal{W} \subseteq \Lambda$ mod is a wide subcategory if it is abelian and extension closed. We let wide $(\Lambda)$ denote the poset of wide subcategories of $\Lambda$-mod, partially ordered by inclusion. It is easy to see that the intersection of two wide subcategories is also a wide subcategory, and the zero subcategory (resp., $\Lambda$-mod) is the unique minimal (resp., unique maximal) element of $\operatorname{wide}(\Lambda)$. Thus, if $\Lambda$ is representation-finite, the poset wide $(\Lambda)$ is a lattice.

THEOREM 7.1. For any tree T, we have the following isomorphisms of posets:

$$
\begin{aligned}
\operatorname{NCP}(T) & \longrightarrow \operatorname{wide}\left(\Lambda_{T}\right) \\
\boldsymbol{B} & \longmapsto \operatorname{add}\left(\oplus_{s_{u}} M(u): s_{u} \in \overline{\operatorname{Seg}(\boldsymbol{B})}\right) .
\end{aligned}
$$

Proof. The map is order-preserving because $\mathbf{B}_{1} \leq \mathbf{B}_{2}$ in $\operatorname{NCP}(T)$ if and only if $\overline{\operatorname{Seg}\left(\mathbf{B}_{1}\right)} \subseteq \overline{\operatorname{Seg}\left(\mathbf{B}_{2}\right)}$ in $\Psi(\overrightarrow{F G}(T))$. Thus, it is enough to show that this map defines a wide subcategory and has an order-preserving inverse.

We now show that $\mathcal{W}:=\operatorname{add}\left(\oplus_{s_{u}} M(u): s_{u} \in \overline{\operatorname{Seg}(\mathbf{B})}\right) \in \operatorname{wide}\left(\Lambda_{T}\right)$ by showing that it equals a category that is known to be wide. Consider the category $\mathcal{W}^{\prime} \subseteq \Lambda_{T}$-mod consisting of objects $M \in \Lambda_{T}$-mod with a filtration $0=M_{0} \subseteq M_{1} \subseteq \cdots \subseteq M_{k}=M$ where for each $i \in\{1, \ldots, k\}$ there exists $M\left(u^{i}\right) \in\left\{M(u): s_{u} \in \operatorname{Seg}(\mathbf{B})\right\}$ such that $M_{i} / M_{i-1}=M\left(u^{i}\right)$. By Lemma 7.2 and Lemma 5.2, the set $\left\{M(u): s_{u} \in \operatorname{Seg}(\mathbf{B})\right\}$ consists of pairwise orthogonal points of $\Lambda_{T}$-mod in the language of [35]. Therefore, by [35, Theorem 1.2], the category $\mathcal{W}^{\prime} \in$ wide $\left(\Lambda_{T}\right)$.

To show that $\mathcal{W} \subseteq \mathcal{W}^{\prime}$, it is enough to show that if $M(v), M(w) \in\left\{M(u): s_{u} \in \overline{\operatorname{Seg}(\mathbf{B})}\right\}$ and they appear in an extension $0 \rightarrow M(v) \rightarrow M(v \leftarrow w) \rightarrow M(w) \rightarrow 0$, then $M(v \leftarrow w) \in$ $\left\{M(u): s_{u} \in \overline{\operatorname{Seg}(\mathbf{B})}\right\}$. This follows from the fact that $s_{v \leftarrow w}=s_{v} \circ s_{w}$ and $\overline{\operatorname{Seg}(\mathbf{B})}$ is closed.

Lastly, we show that $\mathcal{W}^{\prime} \subseteq \mathcal{W}$ by showing that every indecomposable object of $\mathcal{W}^{\prime}$ belongs to $\mathcal{W}$. Let $M(w) \in$ ind $\left(\mathcal{W}^{\prime}\right)$ and let $0=M_{0} \subseteq M_{1} \subseteq \cdots \subseteq M_{k}=M(w)$ be a filtration witnessing that $M(w) \in \mathcal{W}^{\prime}$. We show by induction that each $M_{i} \in \mathcal{W}$. This is obvious for $M_{1}$ so we assume that $M_{i} \in \mathcal{W}$ and prove that $M_{i+1} \in \mathcal{W}$.

Since $M_{i} \in \mathcal{W}$ and by the properties of the filtration of $M(w)$, we can write $M_{i}=\oplus_{r=1}^{\ell} M\left(u^{(r)}\right)$ where $M\left(u^{(r)}\right) \in\left\{M(u): s_{u} \in \overline{\operatorname{Seg}(\mathbf{B})}\right\}$ for all $r \in\{1, \ldots, \ell\}$ and 
$M_{i+1} / M_{i}=M(v)$ where $M(v) \in\left\{M(u): s_{u} \in \operatorname{Seg}(\mathbf{B})\right\}$. Moreover, since $M_{i}$ is submodule of $M(w), \operatorname{supp}\left(M\left(u^{(r)}\right)\right) \cap \operatorname{supp}\left(M\left(u^{\left(r^{\prime}\right)}\right)\right)=\emptyset$ for any distinct $r, r^{\prime} \in\{1, \ldots, \ell\}$. Since $0 \rightarrow$ $\oplus_{r=1}^{\ell} M\left(u^{(r)}\right) \rightarrow M_{i+1} \rightarrow M(v) \rightarrow 0$ is an extension and $M_{i+1}$ is a submodule of $M(w)$, we see that $M_{i+1}$ is a direct sum of submodules $M\left(w^{\prime}\right) \subseteq M(w)$ with pairwise disjoint supports. Furthermore, each segment $s_{W^{\prime}}$ is the composition of segments in $\left\{s_{u^{(r)}}, s_{v}\right\}_{1 \leq r \leq \ell}$. Since $\overline{\operatorname{Seg}(\mathbf{B})}$ is closed, each summand of $M_{i+1}$ belongs to $\mathcal{W}$. We obtain that $M_{i+1} \in \mathcal{W}$. Since $\mathcal{W}=\mathcal{W}^{\prime}$, we know that $\mathcal{W} \in \operatorname{wide}\left(\Lambda_{T}\right)$.

We now claim that the map $\omega$ : wide $\left(\Lambda_{T}\right) \longrightarrow \Psi(\overrightarrow{F G}(T))$ defined by

$$
\mathcal{W} \mapsto \mathcal{S}:=\overline{\left\{s_{u}: M(u) \text { is a simple object of } \mathcal{W}\right\}}
$$

is an order-preserving inverse to the map $\Psi(\overrightarrow{F G}(T)) \longrightarrow$ wide $\left(\Lambda_{T}\right)$. Assuming that $\omega(\mathcal{W}) \in \Psi(\overrightarrow{F G}(T))$, it is clear that $\omega$ is an inverse as a map of sets.

Since each simple object of $\mathcal{W}$ corresponds to a segment of $T$, the partition $\mathbf{B}$ of $V^{o}$ that they induce will have segmented-connected blocks. Thus, to prove that $\mathcal{S}=\overline{\operatorname{Seg}(\mathbf{B})}$ and $\mathbf{B} \in \operatorname{NCP}(T)$, it is enough to show that

- any two distinct simple objects $M(u), M(v) \in \mathcal{W}$ have the property that $s_{u}$ and $s_{v}$ are noncrossing and

- any block $B \in \mathbf{B}$ satisfies $\operatorname{Seg}(B) \subseteq\left\{s_{u}: M(u)\right.$ is a simple object of $\left.\mathcal{W}\right\}$.

Note that $\operatorname{Hom}_{\Lambda_{T}}(M(u), M(v))=\operatorname{Hom}_{\Lambda_{T}}(M(v), M(u))=0$, since $M(u)$ and $M(v)$ are simple objects and $\mathcal{W}$ is a wide subcategory.

If $s_{u}$ and $s_{v}$ share an end point, then Lemma 5.4 implies that $s_{u}$ and $s_{v}$ do not agree along a segment. Thus, they are noncrossing in this case. This case also implies that given any block $B \in \mathbf{B}$, we have that $\operatorname{Seg}(B) \subseteq\left\{s_{u}: M(u)\right.$ is a simple object of $\left.\mathcal{W}\right\}$.

If $s_{u}$ and $s_{v}$ are crossing, then, up to reversing the roles of $u$ and $v$, Theorem 4.8 implies that they define a unique nonsplit extension

$$
0 \rightarrow M(u) \rightarrow M\left(u^{(-)} \leftarrow w \leftarrow v^{(+)}\right) \oplus M\left(v^{(-)} \rightarrow w \rightarrow u^{(+)}\right) \rightarrow M(v) \rightarrow 0
$$

where $u=u^{(-)} \leftarrow w \rightarrow u^{(+)}, v=v^{(-)} \rightarrow w \leftarrow v^{(+)}$. Using this description of the strings $u$ and $v$, we notice that there is map $f \in \operatorname{Hom}_{\Lambda_{T}}(M(u), M(v))$ where $\operatorname{im}(f)=M(w)$, a contradiction. Thus, $s_{u}$ and $s_{v}$ are noncrossing.

Next, we show that $\omega$ is order-preserving. Since any two simple objects of $\mathcal{W} \in$ wide $\left(\Lambda_{T}\right)$ correspond to noncrossing segments, the segment defined by any indecomposable object of $\mathcal{W}$ can be expressed as a concatenation of segments corresponding to simple objects of $\mathcal{W}$. That is, the segments of $\omega(\mathcal{W})$ are in bijection with the indecomposable objects of $\mathcal{W}$. Thus, if $\mathcal{W}_{1} \subseteq \mathcal{W}_{2}$, one has $\omega\left(\mathcal{W}_{1}\right) \subseteq \omega\left(\mathcal{W}_{2}\right)$.

Lemma 7.2. Let $\boldsymbol{B} \in N C P(T)$ and let $M(u), M(v)$ be two distinct indecomposable $\Lambda_{T}$-modules whose corresponding segments appear in $\operatorname{Seg}(B)$ and $\operatorname{Seg}\left(B^{\prime}\right)$, respectively, for some blocks $B$ and $B^{\prime}$ of $\boldsymbol{B}$. Then one has $\operatorname{Hom}_{\Lambda_{T}}(M(u), M(v))=0$ and $\operatorname{Hom}_{\Lambda_{T}}(M(v), M(u))=0$.

Proof. First assume $B=B^{\prime}$. Since $M(u)$ and $M(v)$ are distinct, the corresponding segments $s_{u}$ and $s_{v}$ share at most one vertex of $T$. This means $u$ and $v$ are supported on disjoint sets of vertices of $Q_{T}$ so the statement holds. Thus, we can assume that $s_{u} \in \operatorname{Seg}(B)$ and $s_{v} \in \operatorname{Seg}\left(B^{\prime}\right)$ where $B$ and $B^{\prime}$ are distinct blocks of $\mathbf{B}$. Since $\mathbf{B} \in \operatorname{NCP}(T)$, this implies that $s_{u}$ and $s_{v}$ have no common end points.

Let $\gamma_{u}$ and $\gamma_{v}$ be left admissible curves for $s_{u}$ and $s_{v}$, respectively, witnessing that $s_{u}$ and $s_{v}$ are noncrossing. Write $s_{w}=[a, b]$ for the unique maximal segment along which $s_{u}$ and 
$s_{v}$ agree, if it exists, and orient $\gamma_{u}$ and $\gamma_{v}$ from $a$ to $b$. Without loss of generality, we have two cases:

(i) $\operatorname{supp}(M(u)) \subsetneq \operatorname{supp}(M(v))$,

(ii) $\operatorname{supp}(M(v)) \backslash \operatorname{supp}(M(u)) \neq \emptyset$ and $\operatorname{supp}(M(u)) \backslash \operatorname{supp}(M(v)) \neq \emptyset$.

Suppose $\operatorname{supp}(M(u)) \subsetneq \operatorname{supp}(M(v))$. Here $s_{w}=s_{u}$. By Lemma 3.2 (1), with $s_{u}$ playing the role of $t$, we have that $\gamma_{v}$ either turns left at both $a$ and $b$ or it turns right at both $a$ and $b$. This means that either $v=v^{(1)} \leftarrow u \leftarrow v^{(2)}$ or $v=v^{(1)} \rightarrow u \rightarrow v^{(2)}$ for some nonempty strings $v^{(1)}$ and $v^{(2)}$ in $\Lambda_{T}$. Thus, $\operatorname{Hom}_{\Lambda_{T}}(M(u), M(v))=0$ and $\operatorname{Hom}_{\Lambda_{T}}(M(v)$, $M(u))=0$.

Now suppose that $\operatorname{supp}(M(v)) \backslash \operatorname{supp}(M(u)) \neq \emptyset$ and $\operatorname{supp}(M(u)) \backslash \operatorname{supp}(M(v)) \neq \emptyset$. We can assume that $a$ (resp., $b$ ) is an end point of $s_{v}$ (resp., $s_{u}$ ). Thus, we can write $s_{v}=[a, b] \circ s_{v^{\prime}}$ and $s_{u}=s_{u^{\prime}} \circ[a, b]$ for some nonempty segments $s_{v^{\prime}}, s_{u^{\prime}} \in \operatorname{Seg}(T)$. By Lemma 3.2 (2), with $[a, b]$ playing the role of $t$, we have that either $\gamma_{v}$ turns right at $b$ and $\gamma_{u}$ turns left at $a$ or $\gamma_{v}$ turns left at $b$ and $\gamma_{u}$ turns right at $a$. Thus, either $v=w \rightarrow v^{\prime}$ and $u=u^{\prime} \leftarrow w$ or $v=w \leftarrow v^{\prime}$ and $u=u^{\prime} \rightarrow w$. We conclude that $\operatorname{Hom}_{\Lambda_{T}}(M(u), M(v))=0$ and $\operatorname{Hom}_{\Lambda_{T}}(M(v), M(u))=0$.

8. Simple-minded collections. In this section, we interpret noncrossing tree partitions in terms of the representation theory of $\Lambda_{T}$ using simple-minded collections in the bounded derived category of $\Lambda_{T}$, denoted $\mathcal{D}^{b}\left(\Lambda_{T}\right)$. We show that the data of a noncrossing tree partition and its Kreweras complement is equivalent to a certain type of simple-minded collection.

Simple-minded collections were originally used by Rickard [34] in the construction of derived equivalences of symmetric algebras from stable equivalences. A standard example of a simple-minded collection in representation theory is a complete set of nonisomorphic simple $\Lambda$-modules regarded as elements of $\mathcal{D}^{b}(\Lambda)$. Note that any $\Lambda$-module $X$ becomes an element of $\mathcal{D}^{b}(\Lambda)$ by mapping it to the stalk complex concentrated in degree 0 whose degree 0 term is $X$. Additionally, in [27], simple-minded collections were useful in computing spaces of Bridgeland stability conditions [3].

Here we recall some of the definitions we will need in order to study simple-minded collections. For a more complete presentation of the notions of derived categories and triangulated categories, we refer the reader to Chapter 1 of [26].

Let $\Lambda$ be a finite-dimensional $\mathbb{k}$-algebra (or, more generally, a ring). By a complex, we mean a diagram of finitely generated $\Lambda$-modules

$$
X=\cdots \stackrel{d_{X}^{-2}}{\longrightarrow} X^{-1} \stackrel{d_{X}^{-1}}{\longrightarrow} X^{0} \stackrel{d_{X}^{0}}{\longrightarrow} X^{1} \stackrel{d_{X}^{1}}{\longrightarrow} X^{2} \stackrel{d_{X}^{2}}{\longrightarrow} \cdots
$$

that satisfies $d_{X}^{i+1} \circ d_{X}^{i}=0$ for each $i \in \mathbb{Z}$. We say that the $\Lambda$-module $X^{i}$ in the complex $X$ is in degree $i$. We refer to the $\Lambda$-module homomorphisms $d_{X}^{i}: X^{i} \rightarrow X^{i-1}$ as differentials. If the only nonzero module of a complex $X$ is in degree $i$, we say that $X$ is a stalk complex concentrated in degree $i$. Given a complex $X$, it is natural to define the shift of $X$, denoted $X[1]$, where

$$
X[1]=\cdots \stackrel{-d_{X}^{-1}}{\longrightarrow} X^{0} \stackrel{-d_{X}^{0}}{\longrightarrow} X^{1} \stackrel{-d_{X}^{1}}{\longrightarrow} X^{2} \stackrel{-d_{X}^{2}}{\longrightarrow} X^{3} \stackrel{-d_{X}^{3}}{\longrightarrow} \cdots
$$

and where in $X[1]$ the module in degree $i$ is $X^{i+1}$. Now let $f: X \rightarrow Y$ be a morphism of complexes. We define the mapping cone or cone of $f$, denoted Cone $(f)$, to be the componentwise direct sum of complexes 


$$
X[1] \oplus Y=\cdots \stackrel{d_{\text {Cone }(f)}^{-2}}{\longrightarrow} X^{0} \oplus Y^{-1} \stackrel{d_{\text {Cone } f()}^{-1}}{\longrightarrow} X^{1} \oplus Y^{0} \stackrel{d_{\text {Cone }(f)}^{0}}{\longrightarrow} X^{2} \oplus Y^{1} \stackrel{d_{\text {Cone }(f)}^{1}}{\longrightarrow} X^{3} \oplus Y^{2} \stackrel{d_{\text {Cone }(f)}^{2}}{\longrightarrow} \cdots
$$

with differential given by

$$
d_{\mathrm{Cone}(f)}^{i}=\left[\begin{array}{cc}
-d_{X}^{i+1} & 0 \\
f^{i+1} & d_{Y}^{i}
\end{array}\right]
$$

Dually, one defines the cocone of $f$, denoted Cocone $(f)$.

The bounded derived category of $\Lambda$ has objects given by complexes $X$ of $\Lambda$-modules with $X^{i}=0$ when $|i|$ is sufficiently large. We say that two objects $X, Y \in \mathcal{D}^{b}(\Lambda)$ are quasi-isomorphic if there exists a morphism of complexes $\varphi: X \rightarrow Y$ that induces an isomorphism $H^{k}(X) \rightarrow H^{k}(Y)$ for all $k$. Two objects $X, Y \in \mathcal{D}^{b}(\Lambda)$ are isomorphic if and only if there exists a sequence of quasi-isomorphisms

$$
X=X_{1} \stackrel{\varphi_{1}}{\longrightarrow} X_{2} \stackrel{\varphi_{2}}{\longleftarrow} X_{3} \stackrel{\varphi_{3}}{\longrightarrow} \cdots \stackrel{\varphi_{\ell-1}}{\longleftarrow} X_{\ell}=Y
$$

for some $\ell \geq 1$.

The category $\mathcal{D}^{b}(\Lambda)$, which is a triangulated category, also has the property that any triangle is isomorphic to a triangle of the form

$$
X \stackrel{f}{\longrightarrow} Y \longrightarrow \text { Cone }(f) \longrightarrow X[1] .
$$

In addition, one shows that any triangle in $\mathcal{D}^{b}(\Lambda)$ is isomorphic to one of the form

$$
X[-1] \longrightarrow \text { Cocone }(f) \longrightarrow X \stackrel{f}{\longrightarrow} Y .
$$

Morphism spaces between objects in derived categories can be very complicated. However, the objects in the collections we will study turn out to be stalk complexes. In this situation, the problem of understanding morphisms between such objects in $\mathcal{D}^{b}(\Lambda)$ is more tractable, as the following well-known proposition shows. Then

Proposition 8.1. Let $X, Y \in \mathcal{D}^{b}(\Lambda)$ be stalk complexes concentrated in degree 0.

$$
\operatorname{Hom}_{\mathcal{D}^{b}(\Lambda)}(X[i], Y[j])=\operatorname{Ext}_{\Lambda}^{j-i}(X, Y) .
$$

We now give the main definition of this section.

Definition 8.2. Let $\mathcal{C}$ be a triangulated category. A collection $\left\{X_{1}, \ldots, X_{n}\right\}$ of objects of $\mathcal{C}$ is said to be simple-minded if the following hold for any $i, j \in[n]$ :

i) $\operatorname{Hom}_{\mathcal{C}}\left(X_{i}, X_{j}[k]\right)=0$ for any $k<0$,

ii) $\operatorname{Hom}_{\mathcal{C}}\left(X_{i}, X_{j}\right)=\left\{\begin{array}{l}\mathbb{k}: \text { if } i=j \\ 0: \text { otherwise, }\end{array}\right.$

iii) $\mathcal{C}=$ thick $\left\langle X_{1}, \ldots, X_{n}\right\rangle$ (i.e., the smallest triangulated category containing $X_{1}, \ldots, X_{n}$ and closed under taking summands of objects is $\mathcal{C})$. One says that the objects $\left\{X_{1}, \ldots, X_{n}\right\}$ form a thick subcategory of $\mathcal{C}$.

Now let $\Lambda$ be a finite-dimensional $\mathbb{k}$-algebra and consider a simple-minded collection $\left\{X_{1}, \ldots, X_{n}\right\}$ in $\mathcal{D}^{b}(\Lambda)$. If for each $i \in[n]$ one has $H^{k}\left(X_{i}\right)=0$ for any $k \neq 0,-1$, we say the 
collection is two-term. We let 2-smc $(\Lambda)$ denote the set of isomorphism classes of two-term simple-minded collections of $\mathcal{D}^{b}(\Lambda)$.

It turns out that, as the following lemma shows, it is easy to say what objects can appear in a two-term simple minded collection in $\mathcal{D}^{b}\left(\Lambda_{T}\right)$.

Lemma 8.3. Let $\mathcal{X}=\left\{X_{1}, \ldots, X_{n}\right\} \in 2-\operatorname{smc}\left(\Lambda_{T}\right)$. Each $X_{i} \in \mathcal{X}$ is isomorphic to a stalk complex of an indecomposable $\Lambda_{T}$-module concentrated in degree 0 or -1 .

Proof. By [5, Remark 4.11], each $X \in \mathcal{X}$ is isomorphic to a stalk complex of a $\Lambda_{T}$ module concentrated in degree 0 or -1 . Suppose $X \in \mathcal{X}$ is of the form $X \cong M[1]$ where $M \in \Lambda_{T}$-mod. Now we have that

$$
\operatorname{End}_{\Lambda_{T}}(M)=\operatorname{Hom}_{\Lambda_{T}}(M, M)=\operatorname{Hom}_{\mathcal{D}^{b}\left(\Lambda_{T}\right)}(M, M)=\operatorname{Hom}_{\mathcal{D}^{b}\left(\Lambda_{T}\right)}(M[1], M[1])=\mathbb{k}
$$

where the last equality follows from the fact that $\mathcal{X} \in 2-\operatorname{smc}\left(\Lambda_{T}\right)$. Since $\operatorname{End}_{\Lambda_{T}}(M)$ is a local ring, $M$ is indecomposable. The proof is similar when $X \cong M$ for some $M \in \Lambda_{T}$-mod.

From Lemma 8.3, we have that any two-term simple-minded collection $\mathcal{X}=$ $\left\{X_{1}, \ldots, X_{n}\right\}$ in $\mathcal{D}^{b}\left(\Lambda_{T}\right)$ can be regarded as a collection of segments of $T$. We define $\operatorname{Seg}(\mathcal{X})=\left\{s_{1}, \ldots, s_{n}\right\}$ to be this collection where $s_{i} \in \operatorname{Seg}(\mathcal{X})$ corresponds to $X_{i} \in \mathcal{X}$. Moreover, we can write $\operatorname{Seg}(\mathcal{X})=\operatorname{Seg}^{0}(\mathcal{X}) \sqcup \operatorname{Seg}^{-1}(\mathcal{X})$ where

$$
\operatorname{Seg}^{i}(\mathcal{X}):=\left\{s_{j} \in \operatorname{Seg}(\mathcal{X}): X_{j} \text { is concentrated in degree } i\right\}
$$

The simple-minded collection $\mathcal{X}$ also naturally defines a graph lying on $D^{2}$ as follows. Let $\mathcal{S E G}(\mathcal{X})$ be the graph whose vertices are the internal vertices of $T$ and whose edges are admissible curves $\gamma_{i}$ defined by the segments $s_{i} \in \operatorname{Seg}(\mathcal{X})$ up to isotopy fixing the end points of $\gamma_{i}$ where if $s_{i} \in \operatorname{Seg}^{0}(\mathcal{X})$ (resp., $s_{i} \in \operatorname{Seg}^{-1}(\mathcal{X})$ ) then $\gamma_{i}$ is a green (resp., red) admissible curve. By abuse of notation, we will write $\mathcal{S E \mathcal { G }}(\mathcal{X})=\left\{\gamma_{1}, \ldots, \gamma_{n}\right\}$. It will also be useful to define $\mathcal{S} \mathcal{E} \mathcal{G}^{0}(\mathcal{X})$ (resp., $\mathcal{S E} \mathcal{G}^{-1}(\mathcal{X})$ ) to be the subgraph of $\mathcal{S E G}(\mathcal{X})$ consisting of green (resp., red) admissible curves from $\mathcal{S E G}(\mathcal{X})$.

Our next theorem gives a combinatorial classification of the two-term simple-minded collections for the algebras $\Lambda_{T}$. This theorem implies that the data of a noncrossing tree partition paired with its Kreweras complement is equivalent to that of $\mathcal{S E G}(\mathcal{X})$ for a unique $\mathcal{X} \in 2-\operatorname{smc}\left(\Lambda_{T}\right)$.

THEOREM 8.4. There is a bijection $\theta:\{(\boldsymbol{B}, \operatorname{Kr}(\boldsymbol{B}))\}_{\boldsymbol{B} \in N C P(T)} \longrightarrow 2$-smc $\left(\Lambda_{T}\right)$ given by

$$
\begin{aligned}
& (\boldsymbol{B}, \operatorname{Kr}(\boldsymbol{B})) \stackrel{\theta}{\longmapsto}\left\{M(u)[1]: s_{u} \in \operatorname{Seg}(B) \text { where } B \in \boldsymbol{B}\right\} \sqcup\left\{M(v): s_{v} \in \operatorname{Seg}\left(B^{\prime}\right)\right. \\
& \text { where } \left.B^{\prime} \in \operatorname{Kr}(\boldsymbol{B})\right\} .
\end{aligned}
$$

Proof. The image of $\theta$ lies in $2-\operatorname{smc}\left(\Lambda_{T}\right)$ by Lemma 7.2, Lemma 8.10, and Lemma 8.11.

Furthermore, decompose $\operatorname{Seg}^{0}(\mathcal{X})$ and $\operatorname{Seg}^{-1}(\mathcal{X})$ into segment-connected subsets of maximal size as follows:

$$
\operatorname{Seg}^{0}(\mathcal{X})=\bigsqcup_{i=1}^{\ell} \operatorname{Seg}_{i}^{0}(\mathcal{X}) \text { and } \operatorname{Seg}^{-1}(\mathcal{X})=\bigsqcup_{i=1}^{k} \operatorname{Seg}_{i}^{-1}(\mathcal{X})
$$


In Section 8.2, we construct a map $\epsilon: 2-\operatorname{smc}\left(\Lambda_{T}\right) \longrightarrow\{(\mathbf{B}, \operatorname{Kr}(\mathbf{B}))\}_{\mathbf{B} \in \operatorname{NCP}(T)}$ defined by

$$
\mathcal{X} \stackrel{\epsilon}{\longmapsto}\left(\mathbf{B}_{\mathcal{X}}, \operatorname{Kr}\left(\mathbf{B}_{\mathcal{X}}\right)\right)
$$

where $\mathbf{B}_{\mathcal{X}}:=\left\{B_{1}, \ldots, B_{k}\right\}$ and where $B_{i}:=\{$ vertices of $\mathrm{T}$ that are end points of segments in $\left.\operatorname{Seg}_{i}^{-1}(\mathcal{X})\right\}$. It follows from Proposition 8.9 that $\mathbf{B}_{\mathcal{X}} \in \operatorname{NCP}(T)$ and that any block $B_{i}^{\prime}$ in $\operatorname{Kr}\left(\mathbf{B}_{\mathcal{X}}\right)=\left\{B_{1}^{\prime}, \ldots, B_{\ell}^{\prime}\right\}$ satisfies $B_{i}^{\prime}=$ vertices of $T$ that are end points of segments in $\left.\operatorname{Seg}_{i}^{0}(\mathcal{X})\right\}$.

It is easy to see that $\epsilon=\theta^{-1}$. This completes the proof.

8.1. Mutation of simple-minded collections. Here we recall the notion of mutation of simple-minded collections and interpret this as a combinatorial operation on configurations of admissible curves. Our interpretation of mutation will be a key ingredient in the following results. Mutation was first introduced in [28, Section 8.1] for spherical collections and generalized in [27] to Hom-finite, Krull-Schmidt triangulated categories. This notion is defined using the language of approximations, which we now briefly review.

Let $\mathcal{C}$ be an arbitrary category (not necessarily triangulated), and let $\mathcal{A}$ be any subcategory of $\mathcal{C}$. We say that a morphism $f: C \rightarrow A$ where $C \in \mathcal{C}$ and $A \in \mathcal{A}$ is a left $\mathcal{A}$-approximation of $C$ if for any morphism $g: C \rightarrow A^{\prime}$ where $A^{\prime} \in \mathcal{A}$ one has $g=g^{\prime} f$ for some morphism $g^{\prime}: A \rightarrow A^{\prime}$. Dually, one defines the notion of a right $\mathcal{A}$-approximation of $C$. Additionally, we say that $f: C \rightarrow A$ where $C \in \mathcal{C}$ and $A \in \mathcal{A}$ is left minimal morphism if for every morphism $g: A \rightarrow A$ that satisfies $g f=f$ one has that $g$ is an isomorphism. Dually, one defines right minimal morphisms. A morphism $f: C \rightarrow A$ (resp., $f: A \rightarrow C$ ) is a left minimal $\mathcal{A}$-approximation (resp., right minimal $\mathcal{A}$-approximation) if $f$ is left minimal and is a left $\mathcal{A}$-approximation (resp., right minimal and is a right $\mathcal{A}$-approximation).

Let $\mathcal{X}=\left\{X_{1}, \ldots, X_{n}\right\}$ be a simple-minded collection in $\mathcal{D}^{b}(\Lambda)$ where $\Lambda$ is any finitedimensional $\mathbb{k}$-algebra. Let $\operatorname{ext}\left(X_{k}\right)$ denote the extension closure of $X_{k}$ in $\mathcal{D}^{b}(\Lambda)$ (i.e., the smallest subcategory of $\mathcal{D}^{b}(\Lambda)$ that contains $X_{k}$ and is closed under extensions). We define the left mutation of $\mathcal{X}$ at $X_{k}$ to be $\mu_{k}^{+}(\mathcal{X}):=\left\{X_{1}^{+}, \ldots, X_{n}^{+}\right\}$where

$$
X_{i}^{+}:=\left\{\begin{array}{r}
X_{k}[1]: \text { if } i=k \\
\operatorname{Cone}\left(g_{i}^{+}: X_{i}[-1] \rightarrow X_{k, i}\right): \text { if } i \neq k
\end{array}\right.
$$

where $g_{i}^{+}$is a left minimal ext $\left(X_{k}\right)$-approximation. It is known that such approximations exist and that $\mu_{k}^{+}(\mathcal{X})$ is a simple-minded collection in $\mathcal{D}^{b}(\Lambda)$ (see [27, Section 7.2]). Dually, one defines the right mutation of $\mathcal{X}$ at $X_{k}$, denoted $\mu_{k}^{-}(\mathcal{X})$. The resulting collection $\mu_{k}^{-}(\mathcal{X}):=\left\{X_{1}^{-}, \ldots, X_{n}^{-}\right\}$has objects given by

$$
X_{i}^{-}:=\left\{\begin{array}{r}
X_{k}[-1]: \text { if } i=k \\
\operatorname{Cocone}\left(g_{i}^{-}: X_{k, i} \rightarrow X_{i}[1]\right): \text { if } i \neq k
\end{array}\right.
$$

where $g_{i}^{-}$is a right minimal $\operatorname{ext}\left(X_{k}\right)$-approximation. By [27, Proposition 7.6 (a)], we have $\mu_{k}^{-} \mu_{k}^{+}(\mathcal{X})=\mathcal{X}$ and $\mu_{k}^{+} \mu_{k}^{-}(\mathcal{X})=\mathcal{X}$.

REMARK 8.5. Let $\mathcal{X}=\left\{X_{1}, \ldots, X_{n}\right\} \in 2-\operatorname{smc}\left(\Lambda_{T}\right)$. By Lemma 8.3 , we have that $\mu_{k}^{+}(\mathcal{X}) \in 2-\operatorname{smc}\left(\Lambda_{T}\right)$ (resp., $\mu_{k}^{-}(\mathcal{X}) \in 2-\operatorname{smc}\left(\Lambda_{T}\right)$ ) if and only if $X_{k}$ is a stalk complex of an indecomposable concentrated in degree 0 (resp., -1). Using Proposition 4.6, we have that, when performing the mutation $\mu_{k}^{+}$(resp., $\left.\mu_{k}^{-}\right)$on $\mathcal{X}, \operatorname{ext}\left(X_{k}\right)=\operatorname{add}\left(X_{k}\right)$ (resp., $\left.\operatorname{ext}\left(X_{k}\right)=\operatorname{add}\left(X_{k}[1]\right)\right)$. 
Lemma 8.6. Let $\mathcal{X}=\left\{X_{1}, \ldots, X_{n}\right\}=\left\{M\left(u^{(1)}\right)[1], \ldots, M\left(u^{\left(n_{1}\right)}\right)[1]\right\} \sqcup\left\{M\left(v^{(1)}\right), \ldots\right.$, $\left.M\left(v^{\left(n_{2}\right)}\right)\right\} \in 2-\operatorname{smc}\left(\Lambda_{T}\right)$ and $\operatorname{let}_{i}^{+}: X_{i}[-1] \rightarrow X_{k, i}$ and $g_{i}^{-}: X_{k, i} \rightarrow X_{i}[1]$ be approximations used in the mutations $\mu_{k}^{+}(\mathcal{X})$ and $\mu_{k}^{-}(\mathcal{X})$. Then if $X_{k}=M\left(v^{(j)}\right)$, we have

$$
\begin{aligned}
& X_{i}^{+}=\operatorname{Cone}\left(g_{i}^{+}\right) \cong \\
& \begin{array}{c}
M\left(v^{(j)} \leftarrow v^{\left(j^{\prime}\right)}\right): \\
\operatorname{Ext}_{\Lambda_{T}}^{1}\left(X_{i}, X_{k}\right) \neq 0 \text { and } X_{i}=M\left(v^{\left(j^{\prime}\right)}\right) \text { where } \\
\operatorname{supp}\left(M\left(v^{(j)}\right)\right) \cap \operatorname{supp}\left(M\left(v^{\left(j^{\prime}\right)}\right)\right)=\emptyset,
\end{array} \\
& M(w): \operatorname{Hom}_{\Lambda_{T}}\left(X_{i}[-1], X_{k}\right) \neq 0 \text { and } X_{i}=M\left(u^{\left(j^{\prime}\right)}\right)[1] \text { where } \\
& \operatorname{supp}(M(w))=\operatorname{supp}\left(M\left(v^{(j)}\right)\right) \backslash \operatorname{supp}\left(M\left(u^{\left(j^{\prime}\right)}\right)\right) \text { and } \\
& \operatorname{supp}\left(M\left(u^{\left(j^{\prime}\right)}\right)\right) \subseteq \operatorname{supp}\left(M\left(v^{(j)}\right)\right), \\
& M(w)[1]: \operatorname{Hom}_{\Lambda_{T}}\left(X_{i}[-1], X_{k}\right) \neq 0 \text { and } X_{i}=M\left(u^{\left(j^{\prime}\right)}\right)[1] \text { where } \\
& \operatorname{supp}(M(w))=\operatorname{supp}\left(M\left(u^{\left(j^{\prime}\right)}\right)\right) \backslash \operatorname{supp}\left(M\left(v^{(j)}\right)\right) \text { and } \\
& \operatorname{supp}\left(M\left(u^{(j)}\right)\right) \subseteq \operatorname{supp}\left(M\left(v^{\left(j^{\prime}\right)}\right)\right), \\
& X_{i} \text { : otherwise. }
\end{aligned}
$$

If $X_{k}=M\left(u^{(j)}\right)[1]$, we have

$$
\begin{aligned}
& X_{i}^{-}=\operatorname{Cocone}\left(g_{i}^{-}\right) \cong \\
& \qquad \begin{array}{c}
M\left(u^{\left(j^{\prime}\right)} \leftarrow u^{(j)}\right)[1]: \operatorname{Ext}_{\Lambda_{T}}^{1}\left(X_{k}, X_{i}\right) \neq 0 \text { and } X_{i}=M\left(u^{\left(j^{\prime}\right)}\right)[1] \text { where } \\
\operatorname{supp}\left(M\left(u^{(j)}\right)\right) \cap \operatorname{supp}\left(M\left(u^{\left(j^{\prime}\right)}\right)\right)=\emptyset, \\
M(w)[1]: \operatorname{Hom}_{\Lambda_{T}}\left(X_{k}, X_{i}[1]\right) \neq 0 \text { and } X_{i}=M\left(v^{\left(j^{\prime}\right)}\right) \text { where } \\
\operatorname{supp}(M(w))=\operatorname{supp}\left(M\left(u^{(j)}\right)\right) \backslash \operatorname{supp}\left(M\left(v^{\left(j^{\prime}\right)}\right)\right) \text { and } \\
\operatorname{supp}\left(M\left(v^{\left(j^{\prime}\right)}\right)\right) \subseteq \operatorname{supp}\left(M\left(u^{(j)}\right)\right), \\
M(w): \operatorname{Hom}_{\Lambda_{T}}\left(X_{k}, X_{i}[1]\right) \neq 0 \text { and } X_{i}=M\left(v^{\left(j^{\prime}\right)}\right) \text { where } \\
\operatorname{supp}(M(w))=\operatorname{supp}\left(M\left(v^{\left(j^{\prime}\right)}\right)\right) \backslash \operatorname{supp}\left(M\left(u^{(j)}\right)\right) \text { and } \\
\operatorname{supp}\left(M\left(u^{(j)}\right)\right) \subseteq \operatorname{supp}\left(M\left(v^{\left(j^{\prime}\right)}\right)\right), \\
X_{i}: \operatorname{otherwise.}
\end{array}
\end{aligned}
$$

Lemma 8.6 shows how mutation of a two-term simple-minded collection $\mathcal{X}$ of $\mathcal{D}^{b}\left(\Lambda_{T}\right)$ can be understood combinatorially as an operation on admissible curves in $\mathcal{S E G}(\mathcal{X})$. In Figure 8, we illustrate the possible ways that mutation can affect $\mathcal{S E G}(\mathcal{X})$. Lemma 8.6 also shows that $\mu_{k}^{+}(\mathcal{X})$ differs from $\mathcal{X}$ by at most three objects.

Proof of Lemma 8.6. It is easy to see that $X_{k, i}$ is isomorphic to $X_{k}$ or 0 , since $g_{i}^{+}$is a left minimal add $\left(X_{k}\right)$-approximation. Note that the map $g_{i}^{+}$defines the triangle $X_{i}[-1] \stackrel{g_{i}^{+}}{\longrightarrow}$ $X_{k, i} \longrightarrow \operatorname{Cone}\left(g_{i}^{+}\right) \longrightarrow X_{i}$ in $\mathcal{D}^{b}\left(\Lambda_{T}\right)$. This triangle gives rise to the long exact sequence

$$
\begin{aligned}
0 & \longrightarrow H^{-1}\left(\operatorname{Cone}\left(g_{i}^{+}\right)\right) \longrightarrow H^{0}\left(X_{i}[-1]\right) \stackrel{\left(g_{i}^{+}\right)^{*}}{\longrightarrow} H^{0}\left(X_{k, i}\right) \longrightarrow H^{0}\left(\operatorname{Cone}\left(g_{i}^{+}\right)\right) \\
& \longrightarrow H^{1}\left(X_{i}[-1]\right) \longrightarrow 0,
\end{aligned}
$$

which, by Lemma 8.3, vanishes outside of the terms shown. This sequence becomes $0 \longrightarrow H^{-1}\left(\operatorname{Cone}\left(g_{i}^{+}\right)\right) \longrightarrow H^{-1}\left(X_{i}\right) \stackrel{\left(g_{i}^{+}\right)^{*}}{\longrightarrow} H^{0}\left(X_{k, i}\right) \longrightarrow H^{0}\left(\operatorname{Cone}\left(g_{i}^{+}\right)\right) \longrightarrow H^{0}\left(X_{i}\right) \longrightarrow 0$. 

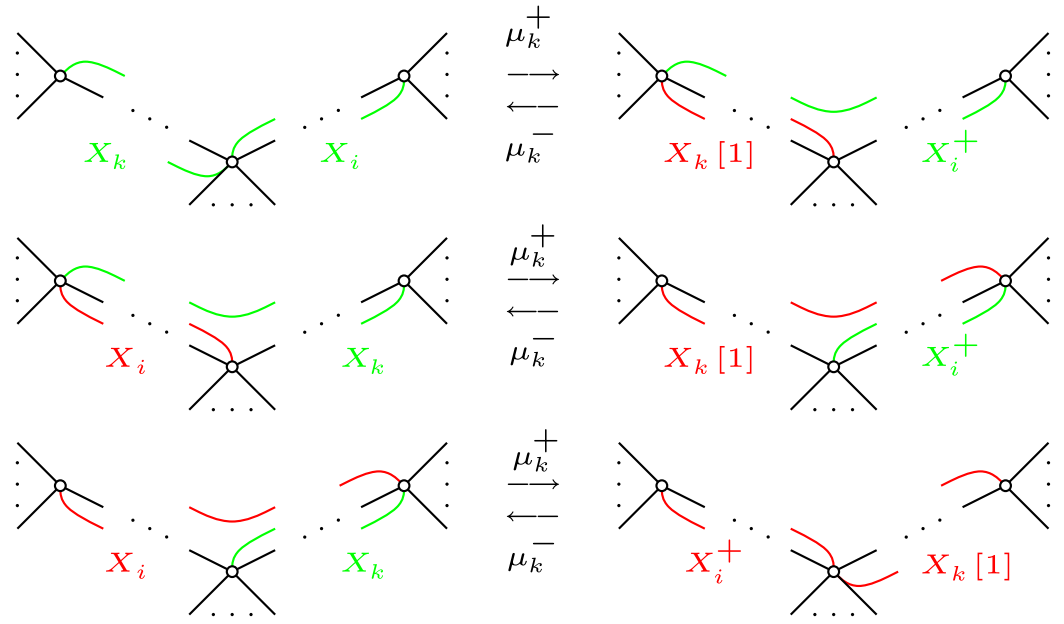

Figure 8. (Color online) The three types of nontrivial transformations.

Now note that since $X_{i}$ is a stalk complex concentrated in degree 0 or -1 , we have the following two cases:

$$
\operatorname{Hom}_{\mathcal{D}^{b}\left(\Lambda_{T}\right)}\left(X_{i}[-1], X_{k, i}\right)= \begin{cases}\operatorname{Ext}_{\Lambda_{T}}^{1}\left(X_{i}, X_{k, i}\right) & : \text { if } H^{0}\left(X_{i}\right)=X_{i}, \\ \operatorname{Hom}_{\Lambda_{T}}\left(X_{i}[-1], X_{k, i}\right): & \text { if } H^{-1}\left(X_{i}\right)=X_{i}\end{cases}
$$

We first consider the case when $H^{0}\left(X_{i}\right)=X_{i}$. By Lemma 5.5, $\operatorname{dim}_{\mathbb{k}} \operatorname{Ext}_{\Lambda_{T}}^{1}\left(X_{i}, X_{k, i}\right) \leq 1$. Suppose that $\operatorname{dim}_{\mathbb{k}} \operatorname{Ext}_{\Lambda_{T}}^{1}\left(X_{i}, X_{k, i}\right)=0$. This means that $g_{i}^{+}: X_{i}[-1] \rightarrow X_{k, i}$ is the zero map. Since $g_{i}^{+}$is a left minimal morphism, this implies that $X_{k, i}=0$. Then the long exact sequence implies that $H^{-1}\left(\operatorname{Cone}\left(g_{i}^{+}\right)\right) \cong H^{-1}\left(X_{i}\right)=0$ and $H^{0}\left(\operatorname{Cone}\left(g_{i}^{+}\right)\right) \cong H^{0}\left(X_{i}\right)=X_{i}$. Thus, Cone $\left(g_{i}^{+}\right) \cong X_{i}$.

Next, suppose that $\operatorname{dim}_{\mathbb{k}} \operatorname{Ext}_{\Lambda_{T}}^{1}\left(X_{i}, X_{k, i}\right)=1$. Since $g_{i}^{+}$is a left minimal morphism, we know that $g_{i}^{+}$is nonzero and thus $X_{k, i}=X_{k}$. Assume that $X_{i}$ is concentrated in degree 0 and write $X_{k}=M\left(v^{(j)}\right), X_{i}=M\left(v^{\left(j^{\prime}\right)}\right)$. Since $\mathcal{X}$ is a simple-minded collection, $\operatorname{Hom}_{\Lambda_{T}}\left(M\left(v^{(j)}\right), M\left(v^{\left(j^{\prime}\right)}\right)\right)=0$ and $\operatorname{Hom}_{\Lambda_{T}}\left(M\left(v^{\left(j^{\prime}\right)}\right), M\left(v^{(j)}\right)\right)=0$. Thus, Theorems 4.7 and 4.8 imply that $0 \rightarrow M\left(v^{(j)}\right) \rightarrow M\left(v^{(j)} \leftarrow v^{\left(j^{\prime}\right)}\right) \rightarrow M\left(v^{\left(j^{\prime}\right)}\right) \rightarrow 0$ is the unique nonsplit extension of $M\left(v^{\left(j^{\prime}\right)}\right)$ by $M\left(v^{(j)}\right)$ up to equivalence of extensions.

Let $M\left(v^{(j)}\right) \longrightarrow M\left(v^{(j)} \leftarrow v^{\left(j^{\prime}\right)}\right) \longrightarrow M\left(v^{\left(j^{\prime}\right)}\right) \stackrel{\xi}{\longrightarrow} M\left(v^{(j)}\right)[1]$ be the triangle in $\mathcal{D}^{b}\left(\Lambda_{T}\right)$ defined by this nonsplit extension where $\xi$ is the class of this extension in $\operatorname{Ext}_{\Lambda_{T}}^{1}\left(M\left(v^{\left(j^{\prime}\right)}\right), M\left(v^{(j)}\right)\right)$. As $\operatorname{dim}_{\mathbb{k}} \operatorname{Ext}_{\Lambda_{T}}^{1}\left(M\left(v^{\left(j^{\prime}\right)}\right), M\left(v^{(j)}\right)\right)=1$, we know that $\xi \neq 0$. Furthermore, we have that $g_{i}^{+}=c \cdot \xi$ for some $c \in \mathbb{k} \backslash\{0\}$. Thus, we have the following isomorphism of triangles in $\mathcal{D}^{b}\left(\Lambda_{T}\right)$ :

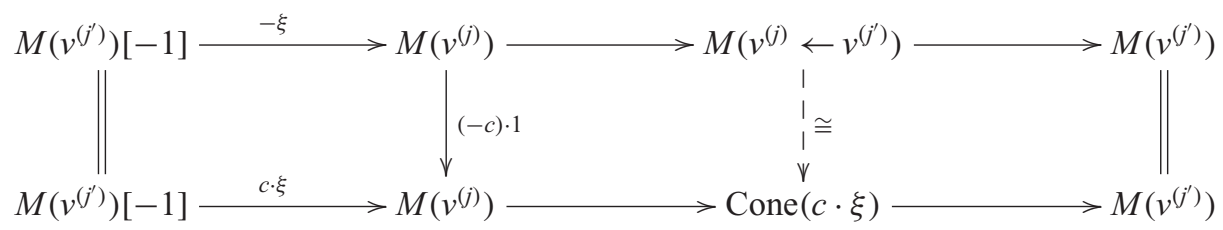

This implies that Cone $\left(g_{i}^{+}\right) \cong M\left(v^{(j)} \leftarrow v^{\left(j^{\prime}\right)}\right)$. 
Next, we consider the case when $H^{-1}\left(X_{i}\right)=X_{i}$. By Lemma 5.3, $\operatorname{dim}_{\mathbb{k}} \operatorname{Hom}_{\Lambda_{T}}$ $\left(X_{i}[-1], X_{k, i}\right) \leq 1$. Suppose that $\operatorname{dim}_{\mathbb{k}} \operatorname{Hom}_{\Lambda_{T}}\left(X_{i}[-1], X_{k, i}\right)=0$. This means that $g_{i}^{+}$: $X_{i}[-1] \rightarrow X_{k, i}$ is the zero map. Since $g_{i}^{+}$is a left minimal morphism, this implies that $X_{k, i}=0$. Then the long exact sequence implies that $H^{-1}\left(\operatorname{Cone}\left(g_{i}^{+}\right)\right) \cong H^{-1}\left(X_{i}\right)=X_{i}$ and $H^{0}\left(\operatorname{Cone}\left(g_{i}^{+}\right)\right) \cong H^{0}\left(X_{i}\right)=0$. Thus, Cone $\left(g_{i}^{+}\right) \cong X_{i}$.

Now suppose that $\operatorname{dim}_{\mathbb{k}} \operatorname{Hom}_{\Lambda_{T}}\left(X_{i}[-1], X_{k, i}\right)=1$. Since $g_{i}^{+}$is a left minimal morphism, we know that $g_{i}^{+}$is nonzero and thus $X_{k, i}=X_{k}$. Thus, if we write $X_{i}[-1]=M\left(u^{\left(j^{\prime}\right)}\right)$ and $X_{k}=M\left(v^{(j)}\right)$, we have that $\operatorname{supp}\left(M\left(u^{\left(j^{\prime}\right)}\right)\right) \cap \operatorname{supp}\left(M\left(v^{(j)}\right)\right) \neq \emptyset$. Furthermore, since $\mathcal{X}$ is a simple-minded collection, we have that

$$
\operatorname{Ext}_{\Lambda_{T}}^{1}\left(M\left(u^{\left(j^{\prime}\right)}\right), M\left(v^{(j)}\right)\right)=\operatorname{Ext}_{\Lambda_{T}}^{1}\left(X_{i}[-1], X_{k}\right)=\operatorname{Hom}_{\mathcal{D}^{b}\left(\Lambda_{T}\right)}\left(X_{i}, X_{k}\right)=0 .
$$

Thus, Theorem 4.8 implies that the segments $s_{u^{(j)}}$ and $s_{v^{\left(j^{\prime}\right)}}$ must share an end point. As $\operatorname{Hom}_{\Lambda_{T}}\left(M\left(u^{\left(j^{\prime}\right)}\right), M\left(v^{(j)}\right)\right) \neq 0$, the two segments must agree along a segment.

We know from Lemma 8.3 that $\operatorname{Cone}\left(g_{i}^{+}\right)$must be isomorphic in $\mathcal{D}^{b}\left(\Lambda_{T}\right)$ to either $M(w)$ or $M(w)[1]$ for some $M(w) \in \operatorname{ind}\left(\Lambda_{T}\right.$-mod) in order to have $\mu_{k}^{+}(\mathcal{X}) \in$ 2 -smc $\left(\Lambda_{T}\right)$. This implies that either $\operatorname{ker}\left(\left(g_{i}^{+}\right)^{*}\right)=0$ or $\operatorname{coker}\left(\left(g_{i}^{+}\right)^{*}\right)=0$. In the former case Cone $\left(g_{i}^{+}\right) \cong M(w)$ where $\operatorname{supp}(M(w))=\operatorname{supp}\left(M\left(v^{(j)}\right)\right) \backslash \operatorname{supp}\left(M\left(u^{\left(j^{\prime}\right)}\right)\right)$. In the latter case Cone $\left(g_{i}^{+}\right) \cong M(w)[1]$ where $\operatorname{supp}(M(w))=\operatorname{supp}\left(M\left(u^{\left(j^{\prime}\right)}\right)\right) \backslash \operatorname{supp}\left(M\left(v^{(j)}\right)\right)$.

The computation of $\operatorname{Cocone}\left(g_{i}^{-}\right)$is similar so we omit it.

8.2. From simple-minded collections to noncrossing tree partitions. In this section, we show how any two-term simple-minded collection gives rise to a noncrossing tree partition paired with its Kreweras complement.

Using left mutation, we can endow $2-\operatorname{smc}\left(\Lambda_{T}\right)$ with a poset structure by regarding it as the transitive closure of the relation $\mathcal{X}_{1} \lessdot \mathcal{X}_{2}$ if and only if $\mathcal{X}_{2}=\mu_{k}^{+}\left(\mathcal{X}_{1}\right)$ for some $k \in$ $[n]$. Perhaps surprisingly, this poset can be understood more globally. In [27, Proposition 7.9] it is shown that the partial order on $\left(2-\operatorname{smc}\left(\Lambda_{T}\right),<\right)$ can be described as follows. If $\mathcal{X}_{1}=\left\{X_{1}^{(1)}, \ldots, X_{n}^{(1)}\right\}, \mathcal{X}_{2}=\left\{X_{1}^{(2)}, \ldots, X_{n}^{(2)}\right\} \in 2-\operatorname{smc}\left(\Lambda_{T}\right)$, then

$$
\mathcal{X}_{1} \leq \mathcal{X}_{2} \text { if and only if } \operatorname{Hom}_{\mathcal{D}^{b}\left(\Lambda_{T}\right)}\left(X_{i}^{(1)}, X_{j}^{(2)}[m]\right)=0
$$

for any $m<0$ and any $i, j \in[n]$. The following proposition shows that the poset $\left(2-\operatorname{smc}\left(\Lambda_{T}\right),<\right)$ has an even richer structure.

Proposition 8.7. The poset $\left(2-\operatorname{smc}\left(\Lambda_{T}\right),<\right)$ is a finite lattice whose unique minimal (resp., maximal) element is $\left\{M(i): i \in\left(Q_{T}\right)_{0}\right\}$ (resp., $\left\{M(i)[1]: i \in\left(Q_{T}\right)_{0}\right\}$ ).

Proof. We will show that $\left(2-\operatorname{smc}\left(\Lambda_{T}\right),<\right)$ is isomorphic to the lattice of torsion-free classes torsf $\left(\Lambda_{T}\right)$. The lattice torsf $\left(\Lambda_{T}\right)$ is finite since $\Lambda_{T}$ is representation-finite.

By [23, Theorem 3.1] and [38, Proposition 2.3], the poset torsf $\left(\Lambda_{T}\right)$ is isomorphic to the poset of bounded $t$-structures $\left(\mathcal{C}_{1}^{\leq 0}, \mathcal{C}_{1}^{\geq 0}\right)$ on $\mathcal{D}^{b}\left(\Lambda_{T}\right)$ that satisfy $\mathcal{C} \leq 0[1] \subseteq \mathcal{C}_{1}^{\leq 0} \subseteq \mathcal{C} \leq 0$ or equivalently, $\mathcal{C}^{\geq 0}[1] \subseteq \mathcal{C}_{1}^{\geq 0} \subseteq \mathcal{C}^{\geq 0}$ where

$$
\begin{aligned}
& \mathcal{C} \leq 0:=\left\{X \in \mathcal{D}^{b}\left(\Lambda_{T}\right): H^{i}(X)=0 \text { for } i>0\right\} \text { and } \\
& \mathcal{C}^{\geq 0}:=\left\{X \in \mathcal{D}^{b}\left(\Lambda_{T}\right): H^{i}(X)=0 \text { for } i \leq-1\right\} .
\end{aligned}
$$

In the latter poset, bounded $t$-structures are partially ordered by inclusion:

$$
\left(\mathcal{C}_{1}^{\leq 0}, \mathcal{C}_{1}^{\geq 0}\right) \leq\left(\mathcal{C}_{2}^{\leq 0}, \mathcal{C}_{2}^{\geq 0}\right) \text { if and only if } \mathcal{C}_{1}^{\geq 0} \subseteq \mathcal{C}_{2}^{\geq 0}, \text { or equivalently, } \mathcal{C}_{1}^{\leq 0} \supset \mathcal{C}_{2}^{\leq 0}
$$


The isomorphism sends a torsion-free class $\mathcal{F}$ and its corresponding torsion class $\mathcal{T}$ to the bounded $t$-structure $\left(\mathcal{C}^{\prime \leq 0}, \mathcal{C}^{\prime \geq 0}\right)$ where

$$
\mathcal{C}_{1}^{\leq 0}:=\left\{X \in \mathcal{D}^{b}\left(\Lambda_{T}\right): H^{i}(X)=0 \text { for } i>0, H^{0}(X) \in \mathcal{T}\right\}
$$

and

$$
\mathcal{C}_{1}^{\geq 0}:=\left\{X \in \mathcal{D}^{b}\left(\Lambda_{T}\right): H^{i}(X)=0 \text { for } i<-1, H^{-1}(X) \in \mathcal{F}\right\} .
$$

Now, by [5, Corollary 4.3] and the remarks following its proof, this poset of bounded $t$-structures is isomorphic to $\left(2-\operatorname{smc}\left(\Lambda_{T}\right),<\right)$.

Remark 8.5 shows that the unique minimal (resp., maximal) element of $\left(2-\operatorname{smc}\left(\Lambda_{T}\right),<\right)$ is $\left\{M(i): i \in\left(Q_{T}\right)_{0}\right\}$ (resp., $\left.\left\{M(i)[1]: i \in\left(Q_{T}\right)_{0}\right\}\right)$. Recall that $M(i)$ is defined as the representation of $Q$ with a one-dimensional vector space assigned to vertex $i$, the zero vector space assigned to all other vertices, and the zero map assigned to all arrows.

Proposition 8.8. Let $\mathcal{X} \in 2$-smc $\left(\Lambda_{T}\right)$. The graph $\mathcal{S E \mathcal { G }}(\mathcal{X})$ is a noncrossing tree (i.e., any two admissible curves in $\mathcal{S E \mathcal { G }}(\mathcal{X})$ are noncrossing in the sense of Lemma 3.2).

Proof. It is clear that $\mathcal{S E G}\left(\left\{M(i): i \in\left(Q_{T}\right)_{0}\right\}\right)$ is a noncrossing tree. By Proposition 8.7, for any $\mathcal{X} \in 2-\operatorname{smc}\left(\Lambda_{T}\right)$ there exists a sequence of left mutations such that $\mathcal{X}=\mu_{i_{k}}^{+} \circ \cdots \circ \mu_{i_{1}}^{+}\left(\left\{M(i): i \in\left(Q_{T}\right)_{0}\right\}\right)$. By Lemma 8.6, we have that if $\mathcal{X}_{2}=\mu_{k}^{+}\left(\mathcal{X}_{1}\right)$ and $\mathcal{S E G}\left(\mathcal{X}_{1}\right)$ is a tree, then $\mathcal{S E G}\left(\mathcal{X}_{2}\right)$ is a tree. Thus, $\mathcal{S E G}(\mathcal{X})$ is a tree.

It remains to prove that if $\mathcal{X}_{2}=\mu_{k}^{+}\left(\mathcal{X}_{1}\right)$ where $\mathcal{X}_{1}, \mathcal{X}_{2} \in 2-\operatorname{smc}\left(\Lambda_{T}\right)$ and $\mathcal{S E G}\left(\mathcal{X}_{1}\right)=$ $\left\{\gamma_{1}, \ldots, \gamma_{n}\right\}$ is noncrossing, then $\mathcal{S E G}\left(\mathcal{X}_{2}\right)=\left\{\gamma_{1}^{+}, \ldots, \gamma_{n}^{+}\right\}$is noncrossing. It is clear that the admissible curves in $\mathcal{S E G}\left(\mathcal{X}_{1}\right) \cap \mathcal{S E \mathcal { G }}\left(\mathcal{X}_{2}\right)$ are noncrossing. Write $\mathcal{X}_{1}=\left\{X_{1}, \ldots, X_{n}\right\}$, $\operatorname{Seg}\left(\mathcal{X}_{1}\right)=\left\{s_{1}, \ldots, s_{n}\right\}$, and $\operatorname{Seg}\left(\mathcal{X}_{2}\right)=\left\{s_{1}^{+}, \ldots, s_{n}^{+}\right\}$. Without loss of generality, we can assume $k=1$ and then $\mathcal{X}_{2}=\left\{X_{1}[1], X_{2}^{+}, \ldots, X_{n}^{+}\right\}$. By Lemma 8.6, $\mathcal{X}_{2}$ differs from $\mathcal{X}_{1}$ in at most three objects. This implies that, without loss of generality, $X_{i}^{+}=X_{i}$ if $i \notin\{1,2,3\}$. Furthermore, the description of mutation in Lemma 8.6 shows that the admissible curves in $\mathcal{S E} \mathcal{G}\left(\mathcal{X}_{2}\right) \backslash \mathcal{S E} \mathcal{G}\left(\mathcal{X}_{1}\right)$ are noncrossing. Thus, it suffices to show any admissible curve from $\mathcal{S E G}\left(\mathcal{X}_{2}\right) \backslash \mathcal{S E G}\left(\mathcal{X}_{1}\right)$ and any admissible curve from $\mathcal{S E} \mathcal{G}\left(\mathcal{X}_{1}\right) \cap \mathcal{S E} \mathcal{G}\left(\mathcal{X}_{2}\right)$ are noncrossing. Note that from our interpretation of mutation in terms of admissible curves (see Figure 8), we see that there is no curve in $\mathcal{S E G}\left(\mathcal{X}_{2}\right) \backslash \mathcal{S E G}\left(\mathcal{X}_{1}\right)$ that crosses one from $\mathcal{S E G}\left(\mathcal{X}_{1}\right) \cap$ $\mathcal{S E G}\left(\mathcal{X}_{2}\right)$ in the sense that the two have a common end point $z(w, F)$ for some corner $(w, F)$ of $T$.

Next, we show that if $\gamma_{\ell}^{+} \in \mathcal{S E G}\left(\mathcal{X}_{2}\right) \backslash \mathcal{S E G}\left(\mathcal{X}_{1}\right)$, then $\gamma_{\ell}^{+}$and any $\gamma_{i}^{+}=\gamma_{i} \in$ $\mathcal{S E G}\left(\mathcal{X}_{1}\right) \cap \mathcal{S E G}\left(\mathcal{X}_{2}\right)$ are noncrossing. Write $s_{\ell}^{+}=s_{w^{(\ell,+)}}$ and $s_{i}=s_{w^{(i)}}$ for some strings $w^{(\ell,+)}$ and $w^{(i)}$ in $\Lambda_{T}$. Let $s_{w}=[a, b]$ be the unique maximal string along which $s_{w^{(\ell,+)}}$ and $s_{w^{(i)}}$ agree and orient $\gamma_{\ell}^{+}$and $\gamma_{i}$ from $a$ to $b$.

Assume $s_{w^{(\ell,+)}}$ and $s_{w^{(i)}}$ share an end point and that $a$ is the shared end point. In this situation, one of $\gamma_{\ell}^{+}$and $\gamma_{i}$ is red admissible and the other is green admissible. We assume $\gamma_{\ell}^{+}$is green admissible and $\gamma_{i}$ is red admissible, and the following argument can be adapted to the case where $\gamma_{\ell}^{+}$is red admissible and $\gamma_{i}$ is green admissible. Since $\mathcal{X}_{2}$ is a simpleminded collection, Definition 8.2 i) implies that

$$
\operatorname{Hom}_{\Lambda_{T}}\left(M\left(w^{(\ell,+)}\right), M\left(w^{(i)}\right)\right)=\operatorname{Hom}_{\mathcal{D}^{b}\left(\Lambda_{T}\right)}\left(M\left(w^{(\ell,+)}\right), M\left(w^{(i)}\right)[1][-1]\right)=0
$$

and so by Lemma 5.4, there is a nonzero morphism $f: M\left(w^{(i)}\right) \rightarrow M\left(w^{(\ell,+)}\right)$. Thus, $w^{(i)}=$ $w \rightarrow u^{(i)}$ and $w^{(\ell,+)}=w \leftarrow u^{(\ell)}$ for some strings $u^{(i)}$ and $u^{(\ell)}$ in $\Lambda_{T}$, one of which may be 
empty. This implies that $\gamma_{\ell}^{+}$turns left at $b$ or $\gamma_{i}$ turns right at $b$. By Lemma 3.2 (c) (with $\gamma_{\ell}^{+}$playing the role of $\gamma$ ), we have that $\gamma_{\ell}^{+}$and $\gamma_{i}$ are noncrossing.

Now suppose that $s_{w^{(\ell,+)}}$ and $s_{w^{(i)}}$ do not share an end point. Assume that $\gamma_{\ell}^{+}$is green admissible and $\gamma_{i}$ is red admissible. The following argument can be adapted to the case when $\gamma_{\ell}^{+}$is red admissible and $\gamma_{i}$ is green admissible. Then since $\mathcal{X}_{2}$ is a simple-minded collection, Definition 8.2 ii) implies that

$$
\operatorname{Ext}_{\Lambda_{T}}^{1}\left(M\left(w^{(\ell,+)}\right), M\left(w^{(i)}\right)\right)=\operatorname{Hom}_{\mathcal{D}^{b}\left(\Lambda_{T}\right)}\left(M\left(w^{(\ell,+)}\right), M\left(w^{(i)}\right)[1]\right)=0 .
$$

By Theorem 4.8 and the structure of $Q_{T}$, we have that one of the following holds:

a) $w^{(i)}=u^{(i, 1)} \leftarrow w \leftarrow u^{(i, 2)}$ and $w^{(\ell,+)}=v^{(\ell, 1)} \stackrel{\alpha}{\rightarrow} w \stackrel{\alpha}{\rightarrow} v^{(\ell, 2)}$ where $u^{(i, 1)}$ and $u^{(i, 2)}$ are nonempty strings and $v^{(\ell, 1)}$ and $v^{(\ell, 2)}$ may be empty strings,

b) $w^{(i)}=u^{(i, 1)} \leftarrow w$ and $w^{(\ell,+)}=v^{(\ell, 1)} \stackrel{\alpha}{\rightarrow} w \stackrel{\beta}{\rightarrow} v^{(\ell, 2)}$ where $u^{(i, 1)}$ and $v^{(\ell, 2)}$ are nonempty strings and $v^{(\ell, 1)}$ may be an empty string,

c) $w^{(i)}=w \leftarrow u^{(i, 2)}$ and $w^{(\ell,+)}=v^{(\ell, 1)} \stackrel{\beta}{\rightarrow} w \stackrel{\alpha}{\rightarrow} v^{(\ell, 2)}$ where $u^{(i, 1)}$ and $v^{(\ell, 1)}$ are nonempty strings and $v^{(\ell, 2)}$ may be an empty string,

$\left.\mathrm{a}^{\prime}\right) w^{(i)}=u^{(i, 1)} \rightarrow w \rightarrow u^{(i, 2)}$ and $w^{(\ell,+)}=v^{(\ell, 1)} \stackrel{\alpha}{\leftarrow} w \stackrel{\alpha}{\leftarrow} v^{(\ell, 2)}$ where $u^{(i, 1)}$ and $u^{(i, 2)}$ are nonempty strings and $v^{(\ell, 1)}$ and $v^{(\ell, 2)}$ may be empty strings,

$\left.\mathrm{b}^{\prime}\right) w^{(i)}=u^{(i, 1)} \rightarrow w$ and $w^{(\ell,+)}=v^{(\ell, 1)} \stackrel{\alpha}{\leftarrow} w \stackrel{\beta}{\leftarrow} v^{(\ell, 2)}$ where $u^{(i, 1)}$ and $v^{(\ell, 2)}$ are nonempty strings and $v^{(\ell, 1)}$ may be an empty string, or

$\left.\mathrm{c}^{\prime}\right) w^{(i)}=w \rightarrow u^{(i, 2)}$ and $w^{(\ell,+)}=v^{(\ell, 1)} \stackrel{\beta}{\leftarrow} w \stackrel{\alpha}{\leftarrow} v^{(\ell, 2)}$ where $u^{(i, 2)}$ and $v^{(\ell, 1)}$ are nonempty strings and $v^{(\ell, 2)}$ may be an empty string.

Here the orientation of the arrows labeled $\beta$ is determined by the fact that $\operatorname{Ext}_{\Lambda_{T}}^{1}\left(M\left(w^{(\ell,+)}\right), M\left(w^{(i)}\right)\right)=0$, while the orientation of the arrows labeled $\alpha$ is determined by the structure of $Q_{T}$. Note that we cannot have $w^{(i)}=u^{(i, 1)} \rightarrow w \leftarrow u^{(i, 2)}$ for some nonempty strings $u^{(i, 1)}$ and $u^{(i, 2)}$, otherwise the structure of $Q_{T}$ implies that

$$
\operatorname{Hom}_{\mathcal{D}^{b}\left(\Lambda_{T}\right)}\left(M\left(w^{(\ell,+)}\right), M\left(w^{(i)}\right)[1][-1]\right)=\operatorname{Hom}_{\Lambda_{T}}\left(M\left(w^{(\ell,+)}\right), M\left(w^{(i)}\right)\right) \neq 0,
$$

and this contradicts that $\mathcal{X}_{2}$ is a simple-minded collection. Using Lemma 3.2, it is straightforward to verify that in each of these cases the admissible curves $\gamma_{\ell}^{+}$and $\gamma_{i}$ are noncrossing.

Finally, assume $s_{w^{(\ell,+)}}$ and $s_{w^{(i)}}$ do not share an end point and $\gamma_{\ell}^{+}$and $\gamma_{i}$ are of the same color. As $\mathcal{X}_{2}$ is a simple-minded collection, we know that $\operatorname{Hom}_{\Lambda_{T}}\left(M\left(w^{(i)}\right), M\left(w^{(\ell,+)}\right)\right)=0$. Thus, $\operatorname{Ext}_{\Lambda_{T}}^{1}\left(M\left(w^{(\ell,+)}\right), M\left(w^{(i)}\right)\right)=0$ by Theorem 4.8. We obtain the same family of cases as in the previous paragraph and, as above, it is routine to verify from these that $\gamma_{\ell}^{+}$and $\gamma_{i}$ are noncrossing.

Proposition 8.9. Let $\mathcal{X} \in 2-\operatorname{smc}\left(\Lambda_{T}\right)$. There exists $\boldsymbol{B}_{\mathcal{X}}=\left\{B_{1}, \ldots, B_{k}\right\} \in N C P(T)$ with Kreweras complement $\operatorname{Kr}\left(\boldsymbol{B}_{\mathcal{X}}\right)=\left\{B_{1}^{\prime}, \ldots, B_{\ell}^{\prime}\right\}$ such that

i) $\operatorname{Seg}^{-1}(\mathcal{X})=\bigsqcup_{i=1}^{k} \operatorname{Seg}\left(B_{i}\right)$

ii) $\operatorname{Seg}^{0}(\mathcal{X})=\bigsqcup_{i=1}^{\ell} \operatorname{Seg}\left(B_{i}^{\prime}\right)$.

Proof. i) Write $\mathcal{S E G}^{-1}(\mathcal{X})=\bigsqcup_{i=1}^{k} \mathcal{S E G}_{i}^{-1}(\mathcal{X})$ where each $\mathcal{S E G}_{i}^{-1}(\mathcal{X})$ is a connected component of $\mathcal{S E G}^{-1}(\mathcal{X})$. Also, let $\operatorname{Seg}_{i}^{-1}(\mathcal{X})$ denote the set of segments defined by $\mathcal{S E G}_{i}^{-1}(\mathcal{X})$.

We claim that any two segments in $\operatorname{Seg}_{i}^{-1}(\mathcal{X})$ either have no common vertices or they agree only at an end point of each. Since $\operatorname{Hom}_{\mathcal{D}^{b}\left(\Lambda_{T}\right)}\left(X_{S}, X_{t}\right)=0$ for any distinct objects in 
$\mathcal{X}$ and since any $\mathcal{S E G}_{i}^{-1}(\mathcal{X})$ is connected, Lemma 5.4 implies that there are no segments in $\operatorname{Seg}_{i}^{-1}(\mathcal{X})$ that share an end point and agree along a segment.

Suppose that $s_{1}, s_{2} \in \operatorname{Seg}_{i}^{-1}(\mathcal{X})$ agree along a segment, but have no common end

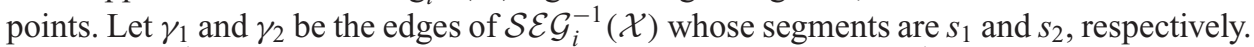
Since $\mathcal{S} \mathcal{E} \mathcal{G}_{i}^{-1}(\mathcal{X})$ is a tree, let $\left(\gamma^{(1)}, \ldots, \gamma^{(r)}\right)$ with $\gamma^{(j)} \in \mathcal{S} \mathcal{E} \mathcal{G}_{i}^{-1}(\mathcal{X})$ denote the unique sequence of edges connecting an end point of $s_{1}$ to an end point of $s_{2}$. Let $\left(s^{(1)}, \ldots, s^{(r)}\right)$ with $s^{(j)} \in \operatorname{Seg}_{i}^{-1}(\mathcal{X})$ denote the sequence of segments defined by $\left(\gamma^{(1)}, \ldots, \gamma^{(r)}\right)$. We assume $s^{(1)}$ (resp., $\left.s^{(r)}\right)$ agrees with $s_{1}$ (resp., $s_{2}$ ) at an end point, and, by the previous paragraph, we can assume that $s^{(j)}$ and $s^{(j+1)}$ agree only at end points for each $j$. Now from the structure of $T$, we have that $s^{(1)}$ agrees with $s_{1}$ along a segment or $s^{(r)}$ agrees with $s_{2}$ along a segment. In either situation we reach a contradiction.

We now have that each $\operatorname{Seg}_{i}^{-1}(\mathcal{X})$ is an inclusion-minimal set of segments. Since $\mathcal{S E G}_{i}^{-1}(\mathcal{X})$ is a connected component of $\mathcal{S E G}^{-1}(\mathcal{X})$, we observe that $\operatorname{Seg}_{i}^{-1}(\mathcal{X})$ is segment-connected. Thus, for each $i \in[k]$, we define

$$
B_{i}:=\left\{v \in T: v \text { is an end point of some segment in } \operatorname{Seg}_{i}^{-1}(\mathcal{X})\right\}
$$

and we have that $\operatorname{Seg}_{i}^{-1}(\mathcal{X})=\operatorname{Seg}\left(B_{i}\right)$. By Proposition 8.8, this implies that $\mathbf{B}_{\mathcal{X}}:=$ $\left\{B_{1}, \ldots, B_{k}\right\} \in \operatorname{NCP}(T)$.

The proof of ii) is similar so we omit it. We remark that the noncrossing tree partition corresponding to $\mathcal{S E G}^{0}(\mathcal{X})=\bigsqcup_{i=1}^{\ell} \mathcal{S E} \mathcal{G}_{i}^{0}(\mathcal{X})$ is defined as $\mathbf{B}^{\prime}:=\left\{B_{1}^{\prime}, \ldots, B_{\ell}^{\prime}\right\}$ where

$$
B_{i}^{\prime}:=\left\{v \in T: v \text { is an end point of some segment in } \operatorname{Seg}_{i}^{0}(\mathcal{X})\right\}
$$

Lastly, we know that $\mathcal{S E G}(\mathcal{X})$ is a noncrossing tree by Proposition 8.8. Furthermore, we have that the green segments in $\operatorname{Seg}^{-1}(\mathcal{X})=\bigsqcup_{i=1}^{k} \operatorname{Seg}\left(B_{i}\right)$ and the red segments in $\operatorname{Seg}^{0}(\mathcal{X})=\bigsqcup_{i=1}^{\ell} \operatorname{Seg}\left(B_{i}^{\prime}\right)$ define a red-green tree. Thus, [21, Corollary 5.12] implies that $\mathbf{B}^{\prime}=\operatorname{Kr}\left(\mathbf{B}_{\mathcal{X}}\right)$.

8.3. From noncrossing tree partitions to simple-minded collections. In this section, we present the last two lemmas needed to show that the image of the map $\theta$, as defined in Theorem 8.4, lies in 2- $\operatorname{smc}\left(\Lambda_{T}\right)$.

LEMMA 8.10. Let $(\boldsymbol{B}, \operatorname{Kr}(\boldsymbol{B})) \in N C P(T)^{2}$ and let $M(u)$ (resp., $\left.M(v)\right)$ be an indecomposable $\Lambda_{T}$-module whose corresponding segment appears in $\operatorname{Seg}(B)$ for some block $B$ of $\boldsymbol{B}$ (resp., of $\operatorname{Kr}(\boldsymbol{B}))$. Then

(1) $\operatorname{Hom}_{\mathcal{D}^{b}\left(\Lambda_{T}\right)}(M(u)[1], M(v)[k])=0$ for any $k \leq 0$,

(2) $\operatorname{Hom}_{\mathcal{D}^{b}\left(\Lambda_{T}\right)}(M(v), M(u)[1][k])=0$ for any $k \leq 0$.

Proof. For each part, we assume that $\mathbf{B}$ is not the top or bottom element of $\operatorname{NCP}(T)$, otherwise the statements hold vacuously. In each part, whenever we assume that $s_{u}=$ $\left[y_{1}, y_{2}\right]$ and $s_{v}=\left[x_{1}, x_{2}\right]$ agree along a segment, we let $s_{w}=[a, b]$ denote the unique maximal segment along which they agree. Furthermore, we let $\gamma_{u}$ and $\gamma_{v}$ be admissible curves for $s_{u}$ and $s_{v}$, respectively, that witness the fact that $s_{u} \in \operatorname{Seg}(B)$ for some block $B$ of $\mathbf{B}$ and $s_{v} \in \operatorname{Seg}\left(B^{\prime}\right)$ for some block $B^{\prime}$ of $\operatorname{Kr}(\mathbf{B})$, and orient this curves from $a$ to $b$.

(1) We have that $\operatorname{Hom}_{\mathcal{D}^{b}\left(\Lambda_{T}\right)}(M(u)[1], M(v)[k])=\operatorname{Ext}_{\Lambda_{T}}^{k-1}(M(u), M(v))=0$, since $k-1 \leq-1$.

(2) Since $\operatorname{Hom}_{\mathcal{D}^{b}\left(\Lambda_{T}\right)}(M(v), M(u)[1][k])=\operatorname{Ext}_{\Lambda_{T}}^{k+1}(M(v), M(u))=0$ for $k \leq-2$, it is enough to show that $\operatorname{Hom}_{\Lambda_{T}}(M(v), M(u))=0$ and $\operatorname{Ext}_{\Lambda_{T}}^{1}(M(v), M(u))=0$. 
We first show that $\operatorname{Hom}_{\Lambda_{T}}(M(v), M(u))=0$. Suppose that $s_{v}$ and $s_{u}$ have no common end points. We claim that $v:=\left\{\left\{x_{1}, x_{2}\right\},\left\{y_{1}, y_{2}\right\},\{i\}: i \in V^{o} \backslash\left\{x_{1}, x_{2}, y_{1}, y_{2}\right\}\right\}$ is a noncrossing tree partition. Since $\gamma_{v}$ and $\gamma_{u}$ do not cross and since $s_{v}$ and $s_{u}$ have no common end points, we can replace $\gamma_{v}$ with a red admissible curve $\gamma_{v}^{\prime}$ representing $s_{v}$ that does not cross $\gamma_{u}$. Thus, $v \in \operatorname{NCP}(T)$. Now by Lemma 7.2, we have that $\operatorname{Hom}_{\Lambda_{T}}(M(v), M(u))=0$.

Now suppose the segments $s_{u}$ and $s_{v}$ share an end point. Since $s_{u} \in \operatorname{Seg}(B)$ for some block $B$ of $\mathbf{B}$ and $s_{v} \in \operatorname{Seg}\left(B^{\prime}\right)$ for some block $B^{\prime}$ of $\operatorname{Kr}(\mathbf{B})$, they are distinct and thus share exactly one end point. We can assume that $s_{u}$ and $s_{v}$ agree along some segment, otherwise we are done. Since $s_{u}$ and $s_{v}$ agree along $s_{w}$, we must have that $v=v^{\prime} \leftrightarrow w$ and $u=u^{\prime} \leftrightarrow w$ for some strings $u^{\prime}$ and $v^{\prime}$ in $\Lambda_{T}$, at least one of which is nonempty. Assume $a$ is the common end point of $s_{u}$ and $s_{v}$. By Lemma 3.2 (3), with $s_{w}=[a, b]$ playing the role of $t$ and $\gamma_{v}$ playing the role of $\gamma$, we have that $\gamma_{v}$ either turns left at $b$ or $\gamma_{u}$ turns right at $b$. Thus either $v=v^{\prime} \rightarrow w$ and $u=w$ or $v=w$ or $u \leftarrow w$. This implies that $\operatorname{Hom}_{\Lambda_{T}}(M(v), M(u))=0$.

Lastly, we show that $\operatorname{Ext}_{\Lambda_{T}}^{1}(M(v), M(u))=0$. By Proposition 4.5, we can restrict to the situation where $s_{u}$ and $s_{v}$ have at least one common vertex of $T$. By Proposition 4.6, we can assume that if $s_{u}$ and $s_{v}$ have only one vertex in common, then that vertex is an end point of each.

Assume $s_{u}$ and $s_{v}$ agree only at an end point. By Lemma 4.7, $\operatorname{Ext}_{\Lambda_{T}}^{1}(M(v), M(u)) \neq 0$ if and only if there exists an arrow $\alpha \in\left(Q_{T}\right)_{1}$ such that the string $(u \longleftrightarrow v)=(u \stackrel{\alpha}{\longleftarrow} v)$. Since $s_{u} \in B \in \mathbf{B}$ and $s_{v} \in B^{\prime} \in \operatorname{Kr}(\mathbf{B})$, any admissible curve $\gamma_{u}$ (resp., $\gamma_{v}$ ) leaves its end points from their right (resp., left). Thus, the existence of such an arrow $\alpha \in\left(Q_{T}\right)_{1}$ implies that $\gamma_{u}$ and $\gamma_{v}$ leave their common end point from a common corner of $T$, and such a configuration is not allowed.

Now assume $s_{u}$ and $s_{v}$ agree along a segment, but they have no common end points. Now we can write $u=u^{(1)} \leftrightarrow w \leftrightarrow u^{(2)}$ and $v=v^{(1)} \leftrightarrow w \leftrightarrow v^{(2)}$ for some strings $u^{(1)}, u^{(2)}, v^{(1)}$, and $v^{(2)}$ in $\Lambda_{T}$ where

i) $u^{(1)}$ and $u^{(2)}$ are nonempty or

ii) $v^{(1)}$ and $v^{(2)}$ are nonempty or

iii) $u^{(1)}$ and $v^{(2)}$ are nonempty and $u^{(2)}$ and $v^{(1)}$ are empty or

iv) $v^{(1)}$ and $u^{(2)}$ are nonempty and $u^{(1)}$ and $v^{(2)}$ are empty.

Suppose we are in case i). Since $s_{u}$ and $s_{v}$ are noncrossing and since $u^{(1)}$ and $u^{(2)}$ are nonempty, we have from Lemma 3.2 (1) (with $s_{w}$ playing the role of $t$ ) that $\gamma_{u}$ either turns left at $a$ and $b$ or turns right at $a$ and $b$. Thus, $u=u^{(1)} \leftarrow w \leftarrow u^{(2)}$ or $u=u^{(1)} \rightarrow w \rightarrow u^{(2)}$. By Theorem 4.8, we have that $\operatorname{Ext}_{\Lambda_{T}}^{1}(M(v), M(u))=0$. In case ii), the analogous arguments shows that $v=v^{(1)} \leftarrow w \leftarrow v^{(2)}$ or $v=v^{(1)} \rightarrow w \rightarrow v^{(2)}$. Thus, Theorem 4.8 implies that $\operatorname{Ext}_{\Lambda_{T}}^{1}(M(v), M(u))=0$.

Suppose we are in case iii). We have from Lemma 3.2 (2) (with $s_{w}$ playing the role of $t, \gamma_{v}$ playing the role of $\gamma$, and $\gamma_{u}$ playing the role of $\gamma^{\prime}$ ) that either $\gamma_{v}$ turns left at $b$ and $\gamma_{u}$ turns right at $a$ or $\gamma_{v}$ turns right at $b$ and $\gamma_{u}$ turns left at $a$. This implies that either $u=u^{(1)} \leftarrow w$ and $v=w \rightarrow v^{(2)}$ or $u=u^{(1)} \rightarrow w$ and $v=w \leftarrow v^{(2)}$. By Theorem 4.8, we have that $\operatorname{Ext}_{\Lambda_{T}}^{1}(M(v), M(u))=0$. The analogous argument can be used in case (iv).

LeMma 8.11. Let $(\boldsymbol{B}, \operatorname{Kr}(\boldsymbol{B})) \in N C P(T)^{2}$. Then the objects

$$
\left\{M(u)[1]: s_{u} \in \operatorname{Seg}(B) \text { where } B \in \boldsymbol{B}\right\} \sqcup\left\{M(v): s_{v} \in \operatorname{Seg}\left(B^{\prime}\right) \text { where } B^{\prime} \in \operatorname{Kr}(\boldsymbol{B})\right\} \subseteq \mathcal{D}^{b}\left(\Lambda_{T}\right)
$$

form a thick subcategory of $\mathcal{D}^{b}\left(\Lambda_{T}\right)$. 
Proof. Let $\mathscr{T}$ denote the smallest triangulated category that contains the objects in the statement of the lemma and that is closed under taking summands of its objects. Note that $M(u) \in \mathscr{T}$ for each $s_{u} \in \operatorname{Seg}(B)$ where $B \in \mathbf{B}$ because $\mathscr{T}$ is closed under taking shifts of objects. Since $\left\{M(i): i \in\left(Q_{T}\right)_{0}\right\}$ is a simple-minded collection, it is enough to show that every indecomposable $\Lambda_{T}$-module belongs to $\mathscr{T}$. To do so, we use what we call admissible sequences of segments.

We say $\left(s_{u^{(1)}}, \ldots, s_{u^{(k)}}\right)$ is an admissible sequence of segments for $s=[a, b]$ if the following hold:

i) $M\left(u^{(i)}\right) \in \mathscr{T}$ for each $i \in[k]$,

ii) segments $s_{u^{(i)}}$ and $s_{u^{(i)}}$ share an end point for each $i \in[k-1]$,

iii) vertex $a$ (resp., $b$ ) is an end point of $s_{1}$ (resp., $s_{k}$ ).

Observe that every segment $s=[a, b]$ has an admissible sequence of segments $\left(s_{u^{(1)}}, \ldots, s_{u^{(k)}}\right)$ of length at most $n$ given by the sequence of segments connecting $a$ and $b$ in the red-green tree defined by $(\mathbf{B}, \operatorname{Kr}(\mathbf{B}))$. We remark that the vertices $a^{\prime}$ and $b^{\prime}$ of $T$ that are the end points shared by $s_{u^{(i)}}$ and $s_{u^{(i+1)}}$ and by $s_{u^{(j)}}$ and $s_{u^{(j+1)}}$, respectively, define a segment $\left[a^{\prime}, b^{\prime}\right] \in \operatorname{Seg}(T)$ for any $i$ and $j$ satisfying $1 \leq i<j<k$. This follows from the fact that $s=[a, b] \in \operatorname{Seg}(T)$.

We prove that if every $s_{u} \in \operatorname{Seg}(T)$ with an admissible sequence $\left(s_{u^{(1)}}, \ldots, s_{u^{(k)}}\right)$ has the property that $M(u) \in \mathscr{T}$, then every $s_{v} \in \operatorname{Seg}(T)$ with an admissible sequence $\left(s_{v^{(1)}}, \ldots, s_{v^{(k+1)}}\right)$ has $M(v) \in \mathscr{T}$. If $s_{u} \in \operatorname{Seg}(T)$ has an admissible sequence $\left(s_{u^{(1)}}\right)$, then $s_{u}=s_{u^{(1)}}$ and so $M(u) \in \mathscr{T}$.

Now assume that every $s_{u} \in \operatorname{Seg}(T)$ with an admissible sequence $\left(s_{u^{(1)}}, \ldots, s_{u^{(k)}}\right)$ has the property that $M(u) \in \mathscr{T}$. Let $s_{v}=[a, b] \in \operatorname{Seg}(T)$ and let $\left(s_{v^{(1)}}, \ldots, s_{v^{(k+1)}}\right)$ be an admissible sequence for $s_{v}$. Observe that in $\left(s_{v^{(1)}}, \ldots, s_{v^{(k+1)}}\right)$ there exists $i \in[k]$ such that, without loss of generality, $s_{v^{(i)}}$ and $s_{v^{(i+1)}}$ are distinct segments that satisfy one of the following:

- $\operatorname{supp}\left(M\left(v^{(i)}\right)\right) \cap \operatorname{supp}\left(M\left(v^{(i+1)}\right)\right)=\emptyset$ or

- $\operatorname{supp}\left(M\left(v^{(i)}\right)\right) \cap \operatorname{supp}\left(M\left(v^{(i+1)}\right)\right) \neq \emptyset$.

Suppose that $\operatorname{supp}\left(M\left(v^{(i)}\right)\right) \cap \operatorname{supp}\left(M\left(v^{(i+1)}\right)\right)=\emptyset$. Note that $s_{v^{(i)}}$ and $s_{v^{(i+1)}}$ agree only at an end point. By the properties of admissible sequences, this implies that $s_{v^{(i)}} \circ S_{v^{(i+1)}} \in \operatorname{Seg}(T)$. Now we have that up to reversing the roles of $v^{(i)}$ and $v^{(i+1)}$, there is a nonsplit extension $0 \rightarrow M\left(v^{(i)}\right) \rightarrow M\left(v^{(i)} \leftarrow v^{(i+1)}\right) \rightarrow M\left(v^{(i+1)}\right) \rightarrow 0$. This means there is a triangle in $\mathcal{D}^{b}\left(\Lambda_{T}\right)$ given by $M\left(v^{(i)}\right) \rightarrow M\left(v^{(i)} \leftarrow v^{(i+1)}\right) \rightarrow$ $M\left(v^{(i+1)}\right) \rightarrow M\left(v^{(i)}\right)[1]$ so $M\left(v^{(i)} \leftarrow v^{(i+1)}\right) \in \mathscr{T}$. We obtain an admissible sequence $\left(s_{v^{(1)}}, \ldots, s_{v^{(i-1)}}, s_{\left(v^{(i)} \leftarrow v^{(i+1)}\right)}, s_{v^{(i+2)}}, \ldots, s_{v^{(k+1)}}\right)$ for $s_{v}$ of length $k$. By induction, we obtain that $M(v) \in \mathscr{T}$.

Now suppose that $\operatorname{supp}\left(M\left(v^{(i)}\right)\right) \cap \operatorname{supp}\left(M\left(v^{(i+1)}\right)\right) \neq \emptyset$. Since $s_{v^{(i)}}$ and $s_{v^{(i+1)}}$ share an end point, it is easy to see that there is nonzero morphism $f: M\left(v^{(i)}\right) \rightarrow M\left(v^{(i+1)}\right)$ or a nonzero morphism $f: M\left(v^{(i+1)}\right) \rightarrow M\left(v^{(i)}\right)$. Without loss of generality, we assume the former. We obtain a triangle in $\mathcal{D}^{b}\left(\Lambda_{T}\right)$ given by $M\left(v^{(i)}\right) \stackrel{f}{\rightarrow} M\left(v^{(i+1)}\right) \rightarrow \operatorname{Cone}(f) \rightarrow$ $M\left(v^{(i)}\right)[1]$ whose long exact sequence reduces to the following exact sequence:

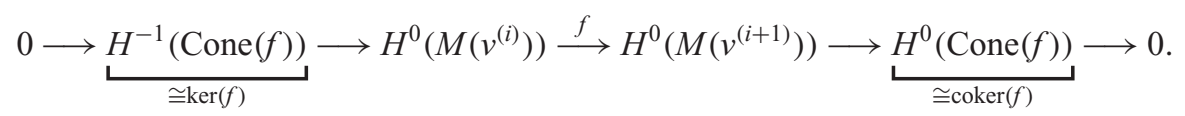

We now have that $\operatorname{Cone}(f)=M\left(w^{(1)}\right)[1] \oplus M\left(w^{(2)}\right)$ where $\operatorname{supp}\left(M\left(w^{(1)}\right)\right)=\operatorname{supp}$ $\left(M\left(v^{(i)}\right)\right) \backslash \operatorname{supp}\left(M\left(v^{(i+1)}\right)\right)$ and $\operatorname{supp}\left(M\left(w^{(2)}\right)\right)=\operatorname{supp}\left(M\left(v^{(i+1)}\right)\right) \backslash \operatorname{supp}\left(M\left(v^{(i)}\right)\right)$. If supp 
$\left(M\left(w^{(1)}\right)\right)=\emptyset\left(\right.$ resp., $\left.\operatorname{supp}\left(M\left(w^{(2)}\right)\right)=\emptyset\right)$, one checks that $\left(s_{v^{(1)}}, \ldots, s_{v^{(i-1)}}, s_{w^{(2)}}, s_{v^{(i+2)}}\right.$, $\left.\ldots, s_{v^{(k+1)}}\right)$ (resp., $\left(s_{v^{(1)}}, \ldots, s_{v^{(i-1)}}, s_{w^{(1)}}, s_{v^{(i+2)}}, \ldots, s_{v^{(k+1)}}\right)$ ) is an admissible sequence for $s_{v}$ of length $k$. By induction, we obtain that $M(v) \in \mathscr{T}$.

Finally, suppose that both $\operatorname{supp}\left(M\left(w^{(1)}\right)\right) \neq \varnothing$ and $\operatorname{supp}\left(M\left(w^{(2)}\right)\right) \neq \emptyset$. Since $\mathscr{T}$ is closed under taking summands of its objects, we have that $M\left(w^{(1)}\right), M\left(w^{(2)}\right) \in \mathscr{T}$. From the properties of admissible sequences, we have that the vertices $a^{\prime}$ and $b^{\prime}$ of $T$ that are the end points shared by $s_{v^{(i-1)}}$ and $s_{v^{(i)}}$ and by $s_{v^{(i+1)}}$ and $s_{v^{(i+2)}}$, respectively, define a segment $\left[a^{\prime}, b^{\prime}\right] \in \operatorname{Seg}(T)$. This implies that $s_{w^{(1)}} \circ s_{w^{(2)}} \in \operatorname{Seg}(T)$. Thus, up to reversing the roles of $w^{(1)}$ and $w^{(2)}$, there is a nonsplit extension $0 \rightarrow M\left(w^{(1)}\right) \rightarrow$ $M\left(w^{(1)} \leftarrow w^{(2)}\right) \rightarrow M\left(w^{(2)}\right) \rightarrow 0$. This extension defines a triangle in $\mathcal{D}^{b}\left(\Lambda_{T}\right)$ given by $M\left(w^{(1)}\right) \rightarrow M\left(w^{(1)} \leftarrow w^{(2)}\right) \rightarrow M\left(w^{(2)}\right) \rightarrow M\left(w^{(1)}\right)[1]$. Thus, $M\left(w^{(1)} \leftarrow w^{(2)}\right) \in \mathscr{T}$. We obtain an admissible sequence $\left(s_{v^{(1)}}, \ldots, s_{v^{(i-1)}}, s_{w^{(1)} \leftarrow w^{(2)}}, s_{v^{(i+2)}}, \ldots, s_{v^{(k+1)}}\right)$ for $s_{v}$ of length $k$. By induction, we obtain that $M(v) \in \mathscr{T}$.

REMARK 8.12. We believe that there exists a generalization of our descriptions of torsion pairs, wide subcategories, and two-term simple-minded collections in greater generality, namely, in the generality of gentle algebras. We are working to find a suitable analogue of the oriented flip graph and the noncrossing tree partitions that will model the combinatorics of these representation-theoretic objects in such generality.

REMARK 8.13. While this manuscript was under review, several authors have studied the combinatorial aspects of the representation theory of gentle algebras; for instance, see $[4,2,31,32]$. In $[2,31]$, it is shown that all gentle algebras can be realized as tiling algebras associated with unpunctured surfaces. In [32], Palu, Pilaud, and Plamondon constructed an analogue of the noncrossing complex that they call the blossoming complex. They also constructed an analogue of the oriented flip graph that they call the non-kissing lattice.

In these papers, the authors have made progress on the problems discussed in Remark 8.12. For posterity, we retain this remark as it appeared in the original version of our manuscript.

9. Classification of c-matrices. We now apply our work to obtain a combinatorial classification of the c-matrices of quivers $Q_{T}$ where the internal vertices of $T$ are all of degree 3. The c-matrices [17] of a quiver $Q$ are related to noncrossing partitions of finite Coxeter groups [33] and many important objects in representation theory [5]. In [5], the cmatrices of quivers were interpreted representation theoretically as certain simple-minded collections in the bounded derived category of a finite-dimensional algebra $\Lambda$. Our result is that c-matrices of $Q_{T}$ are classified by noncrossing tree partitions of $T$ paired with their Kreweras complement.

THEOREM 9.1. Assume that $T$ is a tree whose internal vertices are of degree 3.

(1) The map $\varphi: \operatorname{Seg}(T) \rightarrow \boldsymbol{c}$-vec $(Q)^{+}:=\left\{\right.$positive $\boldsymbol{c}$-vectors of $\left.Q_{T}\right\}$, defined by $s \mapsto$ $\left(a_{1}, \ldots, a_{n}\right) \in \mathbb{Z}_{\geq 0}^{n}$, where $a_{i}:=1$ if the edge corresponding to vertex $i \in\left(Q_{T}\right)_{0}$ appears in $s$ and $a_{i}:=0$ otherwise, is a bijection.

(2) The map $\{(\boldsymbol{B}, \operatorname{Kr}(\boldsymbol{B}))\}_{\boldsymbol{B} \in N C P(T)} \rightarrow \boldsymbol{c}$-mat $(Q)$, defined by sending $(\boldsymbol{B}, \operatorname{Kr}(\boldsymbol{B}))$ to the $\boldsymbol{c}$-matrix $C$ whose negative $\boldsymbol{c}$-vectors are $\{-\varphi(s): s \in \operatorname{Seg}(B)$ where $B \in \boldsymbol{B}\}$ and whose positive c-vectors are $\left\{\varphi(s): s \in \operatorname{Seg}\left(B^{\prime}\right)\right.$ where $\left.B^{\prime} \in \operatorname{Kr}(\boldsymbol{B})\right\}$, is a bijection (see Figure 9). 

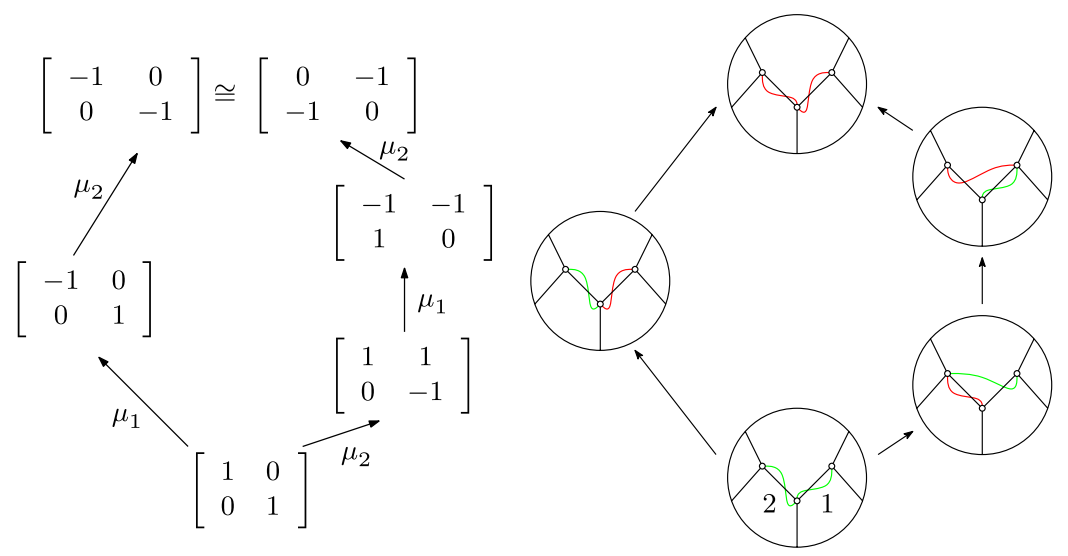

Figure 9. (Color online) The c-matrices of $Q=2 \leftarrow 1$ and the corresponding noncrossing tree partitions with their Kreweras complements.

Proof. (1) By Corollary 4.3, there is a bijection between segments of $T$ and the indecomposable modules of $\Lambda_{T}$. This bijection sends a segment $s$ to a string module $M(w)$ of $\Lambda_{T}$ where $w=w_{1} \leftrightarrow \cdots \leftrightarrow w_{k}$ has the property that each vertex $w_{i} \in\left(Q_{T}\right)_{0}$ corresponds to an edge of $T$ whose vertices both appear in $s$. Now consider the map $\underline{\operatorname{dim}}: \Lambda_{T}$-mod $\rightarrow \mathbb{Z}_{\geq 0}^{n}$. By [11, Theorem 6], the restriction $\underline{\operatorname{dim}}: \operatorname{ind}\left(\Lambda_{T}\right.$-mod $) \rightarrow \mathbf{c}-\operatorname{vec}(Q)^{+}$is a bijection. As the composition $s \mapsto \underline{\operatorname{dim}}(M(w))$ agrees with the map in the assertion, this completes the proof.

(2) By Theorem 8.4, there is a bijective map

$(\mathbf{B}, \operatorname{Kr}(\mathbf{B})) \stackrel{\theta}{\longmapsto}\left\{M(u)[1]: s_{u} \in \operatorname{Seg}(B)\right.$ where $\left.B \in \mathbf{B}\right\} \sqcup\left\{M(v): s_{v} \in \operatorname{Seg}\left(B^{\prime}\right)\right.$ where $\left.B^{\prime} \in \operatorname{Kr}(\mathbf{B})\right\}$

where the latter belongs to $2-\operatorname{smc}\left(\Lambda_{T}\right)$. Define a map $\Phi: 2-\operatorname{smc}\left(\Lambda_{T}\right) \rightarrow \mathbf{c}-\operatorname{mat}(Q)$ by

$$
\left\{X_{1}, \ldots, X_{n}\right\} \mapsto\left\{\underline{\operatorname{dim}}\left(X_{1}\right), \ldots, \underline{\operatorname{dim}}\left(X_{n}\right)\right\}
$$

where $\underline{\operatorname{dim}}: \mathcal{D}^{b}\left(\Lambda_{T}\right) \rightarrow \mathbb{Z}^{n}$ is defined as $\underline{\operatorname{dim}}\left(X_{i}\right):=\sum_{j \in \mathbb{Z}}(-1)^{j} \underline{\operatorname{dim}}\left(X_{i}^{j}\right)$. Recall that, by the assumptions of $T$, the algebras $\Lambda_{T}$ are Jacobian algebras of type $A$. Moreover, for any finitedimensional Jacobian algebra, the map $\Phi$ was shown to be a bijection in [5, Remark 4.19 (a)]. Using the proof of (1), we see that

$(\mathbf{B}, \operatorname{Kr}(\mathbf{B})) \stackrel{\Phi \circ \theta}{\longmapsto}\left\{-\varphi\left(s_{u}\right): s_{u} \in \operatorname{Seg}(B)\right.$ where $\left.B \in \mathbf{B}\right\} \sqcup\left\{\varphi\left(s_{v}\right): s_{v} \in \operatorname{Seg}\left(B^{\prime}\right)\right.$ where $\left.B^{\prime} \in \operatorname{Kr}(\mathbf{B})\right\}$

and the result follows.

AcKnowledgements. Alexander Garver thanks Kiyoshi Igusa, Gregg Musiker, Ralf Schiffler, Sibylle Schroll, and Hugh Thomas for helpful conversations. The authors thank Emily Barnard for useful discussions and Hugh Thomas for noticing the connection between noncrossing tree partitions and wide subcategories. The authors also thank an anonymous referee for their careful comments. Alexander Garver received support from a Research Training Group, RTG Grant no. DMS-1148634. 


\section{REFERENCES}

1. I. Assem, D. Simson and A. Skowroński, Elements of the representation theory of associative algebras: Techniques of representation theory (London Mathematical Society Student Texts 65), vol. 1 (Cambridge University Press, Cambridge, 2006).

2. K. Baur and R. Coelho Simões, A geometric model for the module category of a gentle algebra. arXiv:1803.05802 (2018).

3. T. Bridgeland, Stability conditions on triangulated categories. Ann. of Math. (2) 166(2) (2007), 317-345.

4. T. Brüstle, G. Douville, K. Mousavand, H. Thomas and E. Yıldırım, On the combinatorics of gentle algebras. arXiv:1707.07665 (2017).

5. T. Brüstle and D. Yang, Ordered exchange graphs. Adv. Rep. Theor. Algeb. (ICRA Bielefeld 2012). (2013), 135-193.

6. A. B. Buan, R. Marsh, M. Reineke, I. Reiten and G. Todorov, Tilting theory and cluster combinatorics. Adv. Math. 204(2) (2006), 572-618.

7. P. Caldero, F. Chapoton and R. Schiffler, Quivers with relations arising from clusters $\left(A_{n}\right.$ case). Trans. Amer. Math. Soc. 358(3) (2006), 1347-1364.

8. I. Canacki and S. Schroll, Extensions in Jacobian algebras and cluster categories of marked surfaces. Adv. Math. 313 (2017), 1-49.

9. I. Canakci, D. Pauksztello and S. Schroll, Mapping cones in the bounded derived category of a gentle algebra. arXiv:1609.09688 (2016).

10. I. Canakci, D. Pauksztello and S. Schroll, On extensions for gentle algebras. arXiv:1707.06934 (2017).

11. A. N. Chavez, c-vectors and dimension vectors for cluster-finite quivers. Bull. Lond. Math. Soc. (2013), 1590-1600.

12. R. Coelho Simões and M. J. Parsons, Endomorphism algebras for a class of negative CalabiYau categories. J. Algeb. 491 (2017), 32-57.

13. L. Demonet, Algebras of partial triangulations. arXiv:1602.01592 (2016).

14. L. Demonet, O. Iyama, N. Reading, I. Reiten and H. Thomas, Lattice theory of torsion classes. arXiv:1711.01785 (2017).

15. H. Derksen, J. Weyman and A. Zelevinsky, Quivers with potentials and their representations I: mutations. Selecta Math. 14(1) (2008), 59-119.

16. S. Fomin and A. Zelevinsky, Cluster algebras I: foundations. J. Amer. Math. Soc. 15(2) (2002), 497-529.

17. S. Fomin and A. Zelevinsky, Cluster algebras IV: coefficients. Comp. Math. 143(01) (2007), $112-164$.

18. A. Garver, K. Igusa, J. P. Matherne and J. Ostroff, Combinatorics of exceptional sequences in type A. Electron. J. Combin. (in press).

19. A. Garver and T. McConville, Lattice properties of oriented exchange graphs and torsion classes. Algeb. Rep. Theor. (in press), 1-36.

20. A. Garver and T. McConville, Oriented flip graphs and noncrossing tree partitions. arXiv:1604.06009 (2016).

21. A Garver and T. McConville, Oriented flip graphs of polygonal subdivisions and noncrossing tree partitions. J. Comb. Theor. Ser. A 158 (2018), 126-175.

22. G. Grätzer and F. Wehrung, Lattice theory: special topics and applications. vol. 2 (Springer, Switzerland, 2016).

23. D. Happel, I. Reiten and S. O. Smalø, Tilting in Abelian categories and quasitilted algebras, vol. 575 (American Mathematical Society, Providence, RI, 1996).

24. C. Ingalls and H. Thomas, Noncrossing partitions and representations of quivers. Compos. Math. 145(06) (2009), 1533-1562.

25. O. Iyama, I. Reiten, H. Thomas and G. Todorov, Lattice structure of torsion classes for path algebras. Bull. Lond. Math. Soc. 47(4) (2015), 639-650.

26. M. Kashiwara and P. Schapira, Sheaves on manifolds, vol. 292 (Springer Science \& Business Media, Berlin, Heidelberg, 2013).

27. S. Koenig and D. Yang, Silting objects, simple-minded collections, t-structures and co-tstructures for finite-dimensional algebras. Doc. Math. 19 (2014), 403-438.

28. M. Kontsevich and Y. Soibelman, Stability structures, motivic Donaldson-Thomas invariants and cluster transformations. arXiv:0811.2435 (2008). 
29. F. Marks and J. Št'ovíček, Torsion classes, wide subcategories and localisations. Bull. Lond. Math. Soc. 49(3) (2017), 405-416.

30. G. J. Murphy. Derived equivalence classification of m-cluster tilted algebras of type $A_{n}$. J. Algeb. 323(4) (2010), 920-965.

31. S. Opper, P.-G. Plamondon and S. Schroll, A geometric model for the derived category of gentle algebras, arXiv:1801.09659 (2018).

32. Y. Palu, V. Pilaud and P.-G. Plamondon., Non-kissing complexes and tau-tilting for gentle algebras. Mem. Amer. Math. Soc. (in press).

33. N. Reading, Clusters, Coxeter-sortable elements and noncrossing partitions. Trans. Amer. Math. Soc. 359(12) (2007), 5931-5958.

34. J. Rickard, Equivalences of derived categories for symmetric algebras. J. Algeb. 257(2) (2002), 460-481.

35. C. M. Ringel, Representations of k-species and bimodules. J. Algeb. 41(2) (1976), 269-302.

36. D. Speyer and H. Thomas, Acyclic cluster algebras revisited, in Algebras, quivers and representations (vol. 8 of Abel Symp., Springer, Berlin, Heidelberg, 2013), 275-298.

37. B. Wald and J. Waschbusch, Tame biserial algebras. J. Algeb. 95(2) (1985), 480-500. $663-682$.

38. J. Woolf, Stability conditions, torsion theories and tilting. J. Lond. Math. Soc. 82(3) (2010), 